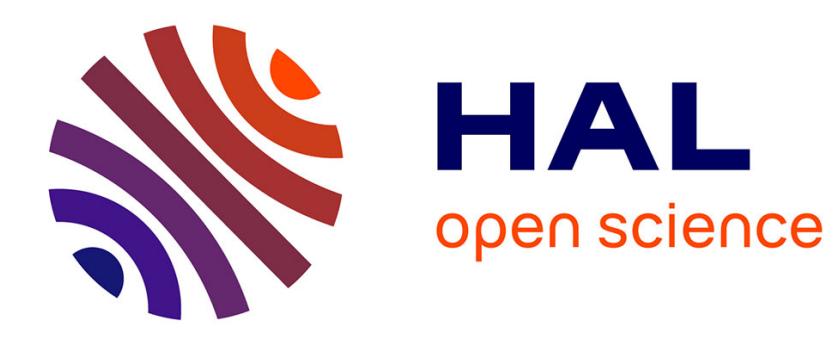

\title{
Existence of Self-similar Energies on Finitely Ramified Fractals \\ Roberto Peirone
}

\section{To cite this version:}

Roberto Peirone. Existence of Self-similar Energies on Finitely Ramified Fractals. 2011. hal00628661

\section{HAL Id: hal-00628661 \\ https://hal.science/hal-00628661}

Preprint submitted on 4 Oct 2011

HAL is a multi-disciplinary open access archive for the deposit and dissemination of scientific research documents, whether they are published or not. The documents may come from teaching and research institutions in France or abroad, or from public or private research centers.
L'archive ouverte pluridisciplinaire HAL, est destinée au dépôt et à la diffusion de documents scientifiques de niveau recherche, publiés ou non, émanant des établissements d'enseignement et de recherche français ou étrangers, des laboratoires publics ou privés. 


\title{
Existence of Self-similar Energies on Finitely Ramified Fractals
}

\author{
Roberto Peirone \\ Università di Roma "Tor Vergata", Dipartimento di Matematica \\ via della Ricerca Scientifica, 00133, Roma, Italy \\ e.mail: peirone@mat.uniroma2.it
}

\begin{abstract}
In this paper, I prove two existence results for regular eigenforms on finitely ramified fractals. The first result shows the existence of a regular eigenform for suitable weights, on fractals with the only assumptions that the boundary cells are separated and the union of the interior cells is connected. This result improves previous results, and works for many of the usually considered finitely ramified fractals. The second result shows the existence of a regular eigenform in the general case of finitely ramified fractals, in the setting considered, for example, in the book of Strichartz. In this more general case, however, the eigenform is not necessarily on the given structure, but on a suitable power of it. In any case, as the fractal generated is the same as the original fractal, the result provides a regular self-similar energy on the given fractal.
\end{abstract}

MSC: $31 \mathrm{C} 25,28 \mathrm{~A} 80,47 \mathrm{H} 10$

\section{Introduction}

The subject of this paper is that of analysis on finitely ramified fractals. The Sierpinski Gasket, the Vicsek Set and the Lindstrøm Snowflake are finitely ramified fractals, while the Sierpinski Carpet is not. The essential reason is that, in the Sierpinski Carpet, some two cells intesect at a segment line and not only at finitely many points. J. Kigami introduced in [3] a general class of finitely ramified fractals, called P.C.F. self-similar sets. The general theory of P.C.F. self-similar sets and many examples can be found in [4]. In this paper, I essentially consider P.C.F. self-similar sets with a mild additional hypothesis - as e.g. considered in [10] - that is, I require that every point in the initial set is a fixed point of one of the contractions defining the fractal. I will only consider connected fractals.

One of the main problems in analysis on finitely ramified fractals is the construction of self-similar Dirichlet forms, i.e. energies, on them, and the basic tool to do this is the construction of a self-similar discrete Dirichlet form defined on a finite subset $V^{(0)}$ of the fractal, which is a sort of boundary of the fractal, but not in a topological sense. Such Dirichlet forms on $V^{(0)}$ are self-similar in the sense that they are eigenforms, that is, eigenfunctions of a special nonlinear operator $\Lambda_{r}$, depending on a set of positive numbers $r_{i}$ (called weights) put on the cells, often called renormalization operator. More precisely, I will call $r$-eigenform an eigenfunction of $\Lambda_{r}$ and G-eigenform (short for generalized eigenform) an $r$-eigenform fo some $r$. In [5], [9] and [6] criteria for the existence of an eigenforms with prescribed weights are discussed. In particular, in [5], T. Lindstrøm proved that there exists an eigenforms on the nested fractals with all weights equal to 1, C. Sabot in [9] proved a rather general criterion, and V. Metz in [6] improved the results in [9]. 
In this paper I consider, instead, the problem whether on a given fractal there exists a set of weights $r$ such that the operator $\Lambda_{r}$ has an eigenform, in other words whether there exist a G-eigenform. In fact, an open problem is

\section{Does a G-eigenform exist on every P.C.F.self-similar set?}

Results about this problem, so far, have been given in [1], in [7] and in [8]. In [1], a method is described that permits to prove the existence of a G-eigenform on fractals with three vertices with some additional, relatively mild, conditions, and on fractals with more than three vertices, but with stronger symmetry assumptions. In [7], the existence of a G-eigenform is proved in the general case of fractals with three vertices. In [8] the existence of a G-eigenform is proved on a relatively general class of fractals, called nicely separated fractals, with an arbitrary number of vertices, and with no symmetries. However, the eigenform turns out to be regular only on a subclass of those fractals, which in any case contains new and nontrivial examples of fractals having a G-eigenform. In [1], the method consists of approximating a collapsed simpler structure in which there is existence and uniqueness of the eigenform, by putting weights tending to infinity on the interior (i.e., containing no vertices) cells. In [7] the existence result follows from a connectedness argument. In [8], the existence of a G-eigenform is proved using an approximation method combined with a fixed point argument. Namely, if we say that a map is stably fixed if it is continuous and maps a suitable nonempty compact and convex set into its interior, we note that a sufficiently close approximation of a stably fixed map has a fixed point. Now, in [8], roughly speaking, $\Lambda_{r}$, or more precisely, a sort of normalization of it, on the given fractal, tends, when some weights tend to infinity (and possibly other weights to 0 ), to a stably fixed map.

In the present paper, I prove two existence results. In the first, proved in Section 4, I merely require that the boundary (i.e., containing a vertex) cells are mutually disjoint and that the set of interior cells is connected, and prove that on such fractals there exists a regular G-eigenform. Such a result improves both the result of [1], in that it does not require any sort of symmetry, and that of [8], in that it does not require any technical condition on the intersection of the boundary cells with the interior cells. The method of proof is similar to that in [8], in the sense that here I use the idea (introduced in [1]) of putting weights tending to infinity on the interior cells, and the corresponding $\Lambda_{r}$ approximate a stably fixed map on a collapsed structure.

The difference is that in [8] the map is a rather technical form of normalization of $\Lambda_{r}$, and this as a natural normalization of $\Lambda_{r}$ (e.g., $\Lambda_{r}$ divided by its norm) fails to be a stably fixed map. In the present paper, instead of using the map $E \mapsto \Lambda_{r}(E)$ with fixed $r$, I use a map of the form $E \mapsto \Lambda_{r(E)}(E)$, where $r$ is a suitable continuous function. A natural normalization of the map obtained by this simple device is in fact a stably fixed map. The proof in the present paper definitely simplifies that in [8].

The second result, proved in Section 8, provides a solution of conjecture $(\mathrm{C})$ in a weak form, that is, I prove that, in the setting considered in the present paper, we have in any case an eigenform, but not necessarily on the given fractal structure, but on a suitable $n$-power of it, for sufficiently large $n$. Roughly speaking, this means that a fractal $K$ can be defined by a set $\left\{\psi_{1}, \ldots, \psi_{k}\right\}$ of one-to-one maps from $K$ to $K$, and we especially investigate the behaviour of $\psi_{i}$ on a special finite subset $V^{(0)}$ of $K$, which I previously 
mentioned in this Introduction. On the other hand, $K$ can also be defined by the set of maps $\left\{\psi_{i_{1}, \ldots, i_{n}}: i_{1}, \ldots, i_{n}=1, \ldots, k\right\}$, for some positive integer $n$. Thus, we can say that such a set is the $n$-power of the original set of maps. The details about such considerations can be found in beginning of Section 3 and in section 5 . Note that, in any case, the result proved in Section 8, provides a self-similar energy on the fractal, the self-similarity being intended with respect to the $n$-power of the set of maps.

The method of proof is a variant of that of the result in Section 4. Any $n$-power with $n>1$ satisfies the condition that the boundary cells are mutually disjoint, but in general there is no reason that it is with connected interior for some $n$. Thus, we have to split the set of interior cells into components. Then, roughly speaking, the idea is that of working separately on every component to get relations on the weights of the components, and gluing suitably such relations. Sections 6-8 are devoted to such a construction.

\section{Notation}

First of all, I recall some notion on graphs. A graph is a pair $(A, G)$ where $A$ is a set and $G$ is a set of subsets of $A$ having precisely two elements. I will also say that $G$ is a graph on $A$. If $\left\{d, d^{\prime}\right\} \in G$, we say that $d$ and $d^{\prime}$ are $G$-close or simply close, and write $d \underset{G}{\sim} d^{\prime}$ or simply $d \sim d^{\prime}$. I will say that a sequence $\left(d_{0}, d_{1}, \ldots, d_{n}\right), n \geq 0$, of elements of $A$ connects $d$ and $d^{\prime}$ (or $d$ to $d^{\prime}$ ) if $d_{0}=d$ and $d_{n}=d^{\prime}$; that it is a path if $d_{i-1} \sim d_{i}$ for every $i=1, \ldots, n$; that it is a weak path if either $d_{i-1}=d_{i}$ or $d_{i-1} \sim d_{i}$, for every $i=1, \ldots, n$; that it is a simple path if it is a path and moreover, the elements $d_{i}$, except possibly for $d_{0}$ and $d_{n}$, are all different. In such a case, we say that a weak path connects $d$ and $d^{\prime}$ in a subset $A^{\prime}$ of $A$ if $d_{i} \in A^{\prime}$ for every $i=1, \ldots, n-1$, and that $d$ and $d^{\prime}$ are connected in $A^{\prime}$ if there exists a path connecting $d$ and $d^{\prime}$ in $A^{\prime}$. Note that by weak path in a set $A$, we will mean that all its elements, but the first and the last, are in $A$, while by weak path contained in $A$ we will mean that all its elements are in $A$. We say that a subset $A^{\prime}$ of $A$ is connected if any two elements of it are connected in $A^{\prime}$. It is easy to verify that the following a), b), c) are equivalent

a) There exists a path connecting $d$ and $d^{\prime}$ in $A^{\prime}$,

b) There exists a weak path connecting $d$ and $d^{\prime}$ in $A^{\prime}$,

c) There exists a simple path connecting $d$ and $d^{\prime}$ in $A^{\prime}$.

Thus, in the previous definitions it is equivalent to consider a path or a weak path or a simple path. If necessary, in the previous definitions, we will stress the relation with $G$ and write for example $G$-connected, $G$-path and so on.

In the sequel, I will use the obvious notation $\mathbb{R}^{A}$ to denote the linear space of the functions from a set $A$ to $\mathbb{R}$ and for every $t \in \mathbb{R}, t_{A}$ will denote the function in $\mathbb{R}^{A}$ taking the value $t$ at every point of $A$. In case $A=\{1, \ldots, n\}$, I will write $\mathbb{R}^{n}$ for $\mathbb{R}^{A}$ as usual, and $t_{(n)}$ for $t_{A}$. I will use the restriction of an element of $\mathbb{R}^{A}$ to a subset $B$ of $A$ in its obvious sense, which can also be interpreted as the projection on $\mathbb{R}^{B}$. I will say that a subset $\mathcal{C}$ of $\mathbb{R}^{A}$ is bounded on $B \subseteq A$ if there exists a constant $K$ such that $\left|x_{b}\right| \leq K$ for all $x \in \mathcal{C}$ and $b \in B$ and that $\mathcal{C}$ is bounded if it is bounded on $A$. Note that if $A$ is finite, then bounded amounts to bounded in the norm. I will denote by $\bar{p}_{A, B}$ such a projection. I will denote 
by $\bar{i}_{A, B}$ the inclusion from $\mathbb{R}^{B}$ to $\mathbb{R}^{A}$ defined by $\bar{i}_{A, B}(f)(a)=\left\{\begin{array}{ll}f(a) & \text { if } a \in B \\ 0 & \text { otherwise }\end{array}\right.$. In the following we denote a finite set $V$ having at least two elements, by $V=\left\{P_{j}: j \in \mathcal{N}\right\}$ where $\mathcal{N}$ is a finite subset of $\mathbb{N} \backslash\{0\}$. Let $J(=J(\mathcal{N}))=\left\{\left\{j_{1}, j_{2}\right\}: j_{1}, j_{2} \in \mathcal{N}, j_{1} \neq j_{2}\right\}$. With a light abuse of notation, we will also write $J=J((V))$. Tipically, $\mathcal{N}=\{1,2, \ldots N\}$, thus $V=\left\{P_{1}, \ldots, P_{N}\right\}, N \geq 2$. Note that in such a case $\# J=M$ where $M=\frac{n(n-1)}{2}$. I will denote by $\mathcal{D}(V)$ or simply $\mathcal{D}$ the set of the Dirichlet forms on $V$, invariant with respect to an additive constant, i.e., the set of the functionals $E$ from $\mathbb{R}^{V}$ into $\mathbb{R}$ of the form

$$
E(u)=\sum_{\left\{j_{1}, j_{2}\right\} \in J} c_{\left\{j_{1}, j_{2}\right\}}(E)\left(u\left(P_{j_{1}}\right)-u\left(P_{j_{2}}\right)\right)^{2}
$$

with $c_{\left\{j_{1}, j_{2}\right\}}(E) \geq 0$. I will denote by $\widetilde{\mathcal{D}}(V)$ or simply $\widetilde{\mathcal{D}}$ the set of the irreducible Dirichlet forms, i.e., $E \in \widetilde{\mathcal{D}}$ if $E \in \mathcal{D}$ and moreover $E(u)=0$ if and only if $u$ is constant. Note that every $E \in \mathcal{D}$ is uniquely determined by its coefficients, namely

$$
c_{\left\{j_{1}, j_{2}\right\}}(E)=\frac{1}{4}\left(E\left(\chi_{\left\{P_{j_{1}}\right\}}-\chi_{\left\{P_{j_{2}}\right\}}\right)-E\left(\chi_{\left\{P_{j_{1}}\right\}}+\chi_{\left\{P_{j_{2}}\right\}}\right)\right)
$$

$\chi_{A}$ denoting the characteristic function of a set $A$ for every $E \in \mathcal{D}$. Thus, we could identify $E \in \mathcal{D}$ with the set of its coefficients in $\mathcal{Q}:=\left[0,+\infty\left[^{J}\right.\right.$. However, as I will also define the set of effective resistances, in order to avoid possible confusion in the interpretation of an element of $\mathcal{Q}$, I will explicitly define this identification as a map. Let $\widetilde{\mathcal{Q}}$ be the set of $q \in \mathcal{Q}$ such that the graph $\mathcal{G} r(=\mathcal{G} r(q))$ on $\mathcal{N}$ defined by $\left\{\left\{j_{1}, j_{2}\right\}: q_{\left\{j_{1}, j_{2}\right\}}>0\right\}$ is connected.

Note that $\widetilde{\mathcal{Q}} \supseteq] 0,+\infty\left[^{J}\right.$. Now, we define the bijection $\bar{I}$ from $\mathcal{Q}$ to $\mathcal{D}$ in the following way:

Given $q \in \mathcal{Q}$ we put $\bar{I}(q)$ to be the element of $\mathcal{D}$ such that $c_{d}(\bar{I}(q))=q_{d}$ for every $d \in J$. In other words,

$$
\bar{I}(q)(u)=\sum_{\left\{j_{1}, j_{2}\right\} \in J} q_{\left\{j_{1}, j_{2}\right\}}\left(u\left(P_{j_{1}}\right)-u\left(P_{j_{2}}\right)\right)^{2} .
$$

Note that $\bar{I}$ maps bijectively $\widetilde{\mathcal{Q}}$ onto $\widetilde{\mathcal{D}}$. We also have $q_{\left\{j_{1}, j_{2}\right\}}=\frac{1}{4}\left(\bar{I}(q)\left(\chi_{\left\{P_{j_{1}}\right\}}-\chi_{\left\{P_{j_{2}}\right\}}\right)-\right.$ $\left.\bar{I}(q)\left(\chi_{\left\{P_{j_{1}}\right\}}+\chi_{\left\{P_{j_{2}}\right\}}\right)\right) \quad \forall\left\{j_{1}, j_{2}\right\} \in J$, . We define

$$
|q|:=\sum_{d \in J} q_{d} \quad \forall q \in \mathcal{Q}, \quad B_{a}=\{q \in \widetilde{\mathcal{Q}}:|q|<a\} .
$$

so that

$$
\bar{I}(q)(u) \leq|q| \bar{I}\left(1_{J}\right)(u)
$$

The problems in this papers, in fact, only concern the sets $\widetilde{\mathcal{D}}$ and $\widetilde{\mathcal{Q}}$. The sets $\mathcal{D}$ and $\mathcal{Q}$ play a merely auxiliary role. I now recall standard results, and, since their proofs are well-known, I will omit them, except for Lemma 2.4 which is less standard. 
Lemma 2.1. For every $q_{n}, q \in \mathcal{Q}$, the following are equivalent

i) $q_{n} \underset{n \rightarrow+\infty}{\longrightarrow} q$

ii) $\bar{I}\left(q_{n}\right) \underset{n \rightarrow+\infty}{\longrightarrow} \bar{I}(q)$ pointwise

iii) $\bar{I}\left(q_{n}\right) \underset{n \rightarrow+\infty}{\longrightarrow} \bar{I}(q)$ uniformly on the compact subsets of $\mathbb{R}^{V}$.

I now recall the notion of the restriction of an element of $\mathcal{D}$. Given $u \in \mathbb{R}^{V^{\prime}}$, put

$$
\begin{gathered}
\mathcal{L}_{V, V^{\prime}}(u)=\left\{v: V \rightarrow \mathbb{R}: v=u \text { on } V^{\prime}\right\}, \\
\mathcal{L}_{V, V^{\prime}}^{\prime}(u)=\left\{v \in \mathcal{L}_{V, V^{\prime}}(u): \min u \leq v \leq \max u\right\} .
\end{gathered}
$$

Given $E \in \mathcal{D}$, a nonempty subset $V^{\prime}$ of $V$ and $u \in \mathbb{R}^{V^{\prime}}$, I denote by

$$
E_{V^{\prime}}(u)=\inf \left\{E(v): v \in \mathcal{L}_{V, V^{\prime}}(u)\right\} .
$$

It is well-known that $\inf \left\{E(v): v \in \mathcal{L}_{V, V^{\prime}}(u)\right\}=\inf \left\{E(v): v \in \mathcal{L}_{V, V^{\prime}}^{\prime}(u)\right\}$ and the infima are in fact minima It follows that for every $E \in \mathcal{D}, \underset{\mathcal{L}_{V, V^{\prime}}(u)}{\min } E$ is attained at some function $H_{V, V^{\prime}, E}(u)$, and we can choose such a function belonging to $\mathcal{L}_{V, V^{\prime}}^{\prime}(u)$. If $E \in \widetilde{\mathcal{D}}$, such a function is unique.

Lemma 2.2. If $E \in \widetilde{\mathcal{D}}$, then there exist positive $c_{1}, c_{2}$ such that

$$
c_{1}(O s c(u))^{2} \leq E(u) \leq c_{2}(O s c(u))^{2} \quad \forall u \in \mathbb{R}^{V}
$$

Lemma 2.3. If $E \in \mathcal{Q}$ and $V^{\prime \prime} \subseteq V^{\prime} \subseteq V$, then $\left(E_{V^{\prime}}\right)_{V^{\prime \prime}}=E_{V^{\prime \prime}}$.

\section{Lemma 2.4.}

i) The map $(q, u) \mapsto \bar{I}(q)(u)$ from $\mathcal{Q}(V) \times \mathbb{R}^{V}$ to $\mathbb{R}$ is continuous

ii) The maps $(q, u) \mapsto H_{V, V^{\prime}, \bar{I}(q)}(u)$ from $\widetilde{\mathcal{Q}}(V) \times \mathbb{R}^{V^{\prime}}$ to $\mathbb{R}^{V}$ and $(q, u) \mapsto \bar{I}(q)_{V^{\prime}}(u)$ from $\mathcal{Q}(V) \times \mathbb{R}^{V^{\prime}}$ to $\mathbb{R}$ are continuous.

Proof. i) and the first statement of ii) are well-known. For the second, let $\left(q_{n}, u_{n}\right) \in \mathcal{Q}(V) \times$ $\mathbb{R}^{V^{\prime}}$ such that $\left(q_{n}, u_{n}\right) \underset{n \rightarrow+\infty}{\longrightarrow}(q, u) \in \mathcal{Q}(V) \times \mathbb{R}^{V^{\prime}}$. Then, $\bar{I}\left(q_{n}\right)_{V^{\prime}}\left(u_{n}\right)=\bar{I}\left(q_{n}\right)\left(v_{n}\right)$ and $v_{n} \in \mathcal{L}_{V, V^{\prime}}^{\prime}\left(u_{n}\right), \quad \bar{I}\left(q_{n}\right)\left(v_{n}\right) \leq \bar{I}\left(q_{n}\right)(v) \quad \forall v \in \mathcal{L}_{V, V^{\prime}}\left(u_{n}\right)$. As $\min u_{n} \leq v_{n} \leq \max u_{n}$, and $A \leq u_{n} \leq B$ for some $A, B \in \mathbb{R}$, taking a subsequence, we can assume that $v_{n} \underset{n \rightarrow+\infty}{\longrightarrow} w \in$ $\mathbb{R}^{V}$. We have $w \in \mathcal{L}_{V, V^{\prime}}(u)$. Moreover, given $\bar{v} \in \mathcal{L}_{V, V^{\prime}}(u)$, taking $v_{n} \in \mathcal{L}_{V, V^{\prime}}\left(u_{n}\right)$ such that $v_{n}=u$ on $V \backslash V^{\prime}$, then $\bar{v}_{n} \underset{n \rightarrow+\infty}{\longrightarrow} \bar{v}$. Therefore, $\bar{I}\left(q_{n}\right)\left(v_{n}\right) \leq \bar{I}\left(q_{n}\right)\left(\bar{v}_{n}\right)$, and, passing to the limit, in view of Lemma 2.4, we get $\lim _{n \rightarrow+\infty} \bar{I}\left(q_{n}\right)_{V^{\prime}}\left(u_{n}\right)=\bar{I}(q)(w) \leq \bar{I}(q)(\bar{v})$, hence 
$\bar{I}\left(q_{n_{h}}\right)_{V^{\prime}}\left(u_{n_{h}}\right) \underset{h \rightarrow+\infty}{\longrightarrow} \bar{I}(q)_{V^{\prime}}(u)$. Thus, every subsequence of $\bar{I}\left(q_{n}\right)_{V^{\prime}}\left(u_{n}\right)$ has a subsequence convergent to $\bar{I}(q)_{V^{\prime}}(u)$, thus $\bar{I}\left(q_{n}\right)_{V^{\prime}}\left(u_{n}\right) \underset{n \rightarrow+\infty}{\longrightarrow} \bar{I}(q)_{V^{\prime}}(u)$.

Lemma 2.5. We have $E_{V^{\prime}} \in \widetilde{\mathcal{D}}\left(V^{\prime}\right)$ for every $E \in \widetilde{\mathcal{D}}(V)$ and $E_{V^{\prime}} \in \mathcal{D}\left(V^{\prime}\right)$ for every $E \in \mathcal{D}(V)$.

Lemma 2.6. If $E \in \widetilde{\mathcal{D}}$, the function $v:=H_{V, V^{\prime}, E}(u)$ is the unique function from $V$ to $\mathbb{R}$ having the following properties:

i) $v=u$ on $V^{\prime}$

ii) $\sum_{j^{\prime} \neq j} c_{\left\{j, j^{\prime}\right\}}\left(v\left(P_{j}\right)-v\left(P_{j^{\prime}}\right)\right)=0$ for every $P_{j} \in V \backslash V^{\prime}$.

Also, $H_{V, V^{\prime}, E}$ is linear on $\mathbb{R}^{V^{\prime}}$, and $H_{V, V^{\prime}, E}(c)=c$ for every constant $c$.

I will now introduce the notion of effective resistances, which will play a key role in the following. Its reciprocal can be also called effective conductivity. The importance of such notions is related to the fact that they are simpler to evaluate in combinations of sets than the actual conductivities $c_{j_{1}, j_{2}}$, e.g. there are the rules of resistances in series or simlar. Given two nonempty disjoint subsets (shortly nptdss) of $V A$ and $B$, we put

$$
\begin{gathered}
\mathcal{L}_{V ; A, B}=\left\{v \in \mathbb{R}^{V}: v(P)=0 \forall P \in A, v(P)=1 \forall P \in B\right\}, \\
\mathcal{L}_{V ; A, B}^{\prime}=\left\{v \in \mathcal{L}_{V ; A, B}: 0 \leq v \leq 1\right\} .
\end{gathered}
$$

and if $q \in \mathcal{Q}$, we recall that the effective resistance $\widehat{R}(q)_{\{A, B\}}$ is defined by

$$
\widehat{R}(q)_{\{A, B\}}=\frac{1}{\bar{I}(q)_{A \cup B}\left(\chi_{B}\right)}=\frac{1}{\inf \left\{\bar{I}(q)(v) \mid v \in \mathcal{L}_{V ; A, B}\right\}} .
$$

Put also

$$
\bar{R}(q)_{\left\{j_{1}, j_{2}\right\}}=\widehat{R}(q)_{\left\{\left\{P_{j_{1}}\right\},\left\{P_{j_{2}}\right\}\right\}}=\frac{1}{\inf \left\{\bar{I}(q)(v) \mid v \in \mathcal{L}_{V ;\left\{P_{j_{1}}\right\},\left\{P_{j_{2}}\right\}}\right\}}
$$

when $\left\{j_{1}, j_{2}\right\} \in J$. This is the most usual case (effective resistance between two points). In the sequel, we will put $(1 / \widehat{R})(q)=(\widehat{R}(q))^{-1}$, and $(1 / \bar{R})(q)=(\bar{R}(q))^{-1}$. In the previous definition we use the convention $\frac{1}{0}=+\infty$. Note that, as, given $v \in \mathbb{R}^{V}$, we have

$$
v \in \mathcal{L}_{V ; A, B} \Longleftrightarrow 1-v \in \mathcal{L}_{V ; B, A}, \quad E(v)=E(1-v) \quad \forall E \in \mathcal{D}
$$

thus the definition of $\widehat{R}(q)_{\{A, B\}}$ is in fact independent of the order of $A$ and $B$. In fact, Also, $\left.\widehat{R}(q)_{\{A, B\}} \in\right] 0,+\infty[$ if $q \in \widetilde{\mathcal{Q}}$. I will know discuss simple properties of effective resistances.

Lemma 2.7. If $q \in \mathcal{Q}$ and $\left\{\bar{j}, \bar{j}^{\prime}\right\} \in J$, then

i) $\bar{R}(q)_{\left\{\bar{j}, \bar{j}^{\prime}\right\}}<+\infty$ if and only if $\bar{j}$ and $\bar{j}^{\prime}$ are connected in $\mathcal{G} r$. 
ii) If $\bar{j}$ and $\bar{j}^{\prime}$ are connected in $\mathcal{G} r$, and $C$ is their component, then

$$
\bar{R}(q)_{\left\{\bar{j}, \bar{j}^{\prime}\right\}}=\bar{R}\left(\left.q\right|_{J(C)}\right)_{\left\{\bar{j}_{, j^{\prime}}\right\}}<+\infty
$$

\section{Lemma 2.8.}

i) The $\operatorname{map}(1 / \bar{R}): \mathcal{Q} \rightarrow\left[0,+\infty\left[^{J}\right.\right.$ is one-to-one and continuous.

ii) The set $\bar{R}(] 0,+\infty\left[^{J}\right)$ is an open set in $] 0,+\infty\left[^{J}\right.$, and $\bar{R}$ is a homeomorphism from ] $0,+\infty\left[{ }^{J}\right.$ onto its image.

iii) The map $\bar{R}$ is a homeomorphism from any bounded subset of $\widetilde{\mathcal{Q}}$ onto its image.

iv) For every $R \in \bar{R}(\widetilde{\mathcal{Q}})$, there exists $U$ neighbourhood of $R$ such that $\bar{R}^{-1}(U)$ is a bounded subset of $\widetilde{\mathcal{Q}}$.

Proof. i) The map $(1 / \bar{R})$ is continuous by Lemma 2.4. Moreover, it is one-to-one on $\widetilde{\mathcal{Q}}$ by [4], Theorem 2.1.12. We are going to prove it is one-to-one on $\mathcal{Q}$, by reducing this case to that on $\mathcal{Q}$, using Lemma 2.7. Namely, Let $q_{1}, q_{2} \in \mathcal{Q}$, and suppose $\bar{R}\left(q_{1}\right)=\bar{R}\left(q_{2}\right)$. We have two possibilities: either $\bar{j}$ and $\bar{j}^{\prime}$ are not connected in $\mathcal{G} r\left(q_{1}\right)$ (or equivalently in $\mathcal{G} r\left(q_{2}\right)$ ) and thus $\left(q_{1}\right)_{\left\{\bar{j}, \bar{j}^{\prime}\right\}}=\left(q_{2}\right)_{\left\{\bar{j}, \bar{j}^{\prime}\right\}}=0$ or $\bar{j}$ and $\bar{j}^{\prime}$ are connected in $\mathcal{G} r\left(q_{1}\right)$. In the latter case, if $C$ denotes their component both in $\mathcal{G} r\left(q_{1}\right)$ and in $\mathcal{G} r\left(q_{2}\right)$, then $\left.\left(q_{1}\right)\right|_{J(C)},\left.\left(q_{2}\right)\right|_{J(C)} \in \widetilde{\mathcal{Q}}\left(V^{\prime}\right)$ where $V^{\prime}:=\left\{P_{j}: j \in C\right\}$, and by Lemma 2.7 again, $\bar{R}\left(\left.\left(q_{1}\right)\right|_{J(C)}\right)=\bar{R}\left(\left.\left(q_{2}\right)\right|_{J(C)}\right)_{\left\{j_{1}, j_{2}\right\}}$ on $J(C)$. Thus, $\left.\left(q_{1}\right)\right|_{J(C)}=\left.\left(q_{2}\right)\right|_{J(C)}$, in particular, $\left(q_{1}\right)_{\left\{\bar{j} \bar{j}^{\prime}\right\}}=\left(q_{2}\right)_{\left\{\bar{j}, \bar{j}^{\prime}\right\}}$. In conclusion, $\left(q_{1}\right)_{\left\{\bar{j}, \bar{j}^{\prime}\right\}}=\left(q_{2}\right)_{\left\{\bar{j}, \bar{j}^{\prime}\right\}}$ for each $\left\{\bar{j}, \bar{j}^{\prime}\right\} \in J$ and i) is proved.

ii) follows from the well known statement that a one-to-one continuous map from an open set in $\mathbb{R}^{n}$ into $\mathbb{R}^{n}$ is open, i.e., sends open sets into open sets.

iii) Let $F$ be a bounded subset of $\widetilde{\mathcal{Q}}$. Then $(1 / \bar{R})$ is continuous and one-to-one, thus a homeomorphism, from the compact set $\bar{F}$ onto its image. By restricting it, $(1 / \bar{R})$ is a homeomorphism from $F$ onto its image.

iv) We have $R_{d} \geq A>0$ for every $d \in J$. Take $U$ neighbourhood of $R$ such that for every $R^{\prime} \in U$ we have $R_{d}^{\prime}>\frac{A}{2}$ for every $d \in J$. For every $q \in \bar{R}^{-1}(U)$ we have $q_{d} \leq \frac{2}{A}$ for every $d \in J$. In fact, if there exists $\left\{j_{1}, j_{2}\right\} \in J$ such that $q_{\left\{j_{1}, j_{2}\right\}}>\frac{2}{A}$, then

$$
\bar{I}(q)(v) \geq q_{\left\{j_{1}, j_{2}\right\}} \quad \forall v \in \mathcal{L}_{V ; P_{j_{1}}, P_{j_{2}}} .
$$

Thus $(1 / \bar{R})(q)_{\left\{j_{1}, j_{2}\right\}}>\frac{2}{A}$, and $\bar{R}(q)_{\left\{j_{1}, j_{2}\right\}}<\frac{A}{2}$, but $\bar{R}(q) \in U$, a contradiction. Moreover, given $R^{\prime} \in U$, we also have $R^{\prime} \in \mathbb{R}^{J}$, hence $R_{d}^{\prime}<+\infty$ for every $d \in J$. Therefore, for every $q \in \bar{R}^{-1}(U)$ and $\left\{j_{1}, j_{2}\right\} \in J$, we have $\bar{R}(q)_{\left\{j_{1}, j_{2}\right\}}<+\infty$, thus $j_{1}$ and $j_{2}$ are connected in $\mathcal{G} r(q)$ by Lemma $2.7 \mathrm{i}$ ), and $q \in \widetilde{\mathcal{Q}}$.

Remark 2.9. If $A B$ are nptdss of $V$ and $q \in \mathcal{Q}$, then $\min \left\{\bar{I}(q)(v): v \in \mathbb{R}^{V}: v(P)=t_{1} \forall P \in A, v(P)=t_{2} \forall P \in B\right\}=\left(t_{1}-t_{2}\right)^{2}(1 / \widehat{R})(q)_{A, B}$. 


\section{The fractal general setting.}

I will now define the fractal setting, which is based on that in [7]. This kind of approach was firstly given in [2]. We define a fractal by giving a fractal triple, i.e., a triple $\left(V^{(0)}, V^{(1)}, \Psi\right)$ where $V^{(0)}$ and $V^{(1)}$ are finite sets with $\# V^{(0)} \geq 2$, and $\Psi$ is a finite set of one-to-one maps from $V^{(0)}$ into $V^{(1)}$ satisfying

$$
V^{(1)}=\bigcup_{\psi \in \Psi} \psi\left(V^{(0)}\right)
$$

We put $V^{(0)}=\left\{P_{1}, \ldots, P_{N}\right\}$, and of course $N \geq 2$. A set of the form $\psi\left(V^{(0)}\right)$ with $\psi \in \Psi$ will be called a cell or a 1-cell. We require that

a) For each $j=1, \ldots, N$ there exists a (unique) map $\psi_{j} \in \Psi$ such that $\psi_{j}\left(P_{j}\right)=P_{j}$, and $\Psi=\left\{\psi_{1}, \ldots, \psi_{k}\right\}$, with $k \geq N$.

b) $P_{j} \notin \psi_{i}\left(V^{(0)}\right)$ when $i \neq j$ (in other words, if $\psi_{i}\left(P_{h}\right)=P_{j}$ with $i=1, \ldots, k, j, h=1, \ldots, N$, then $i=j=h$ ).

c) Any two points in $V^{(1)}$ can be connected by a path whose any edge belongs to a 1-cell, depending of the edge.

Of course, it immediately follows $V^{(0)} \subseteq V^{(1)}$. Let $\left.W=\right] 0,+\infty\left[{ }^{k}\right.$. We put $\widetilde{\mathcal{D}}=\widetilde{\mathcal{D}}\left(V^{(0)}\right)$, and in general we will consider the previous setting with $V=V^{(0)}$. Put $V_{i}=\psi_{i}\left(V^{(0)}\right)$ for each $i=1, \ldots, k$ and put

$$
V(\mathcal{B}):=\bigcup_{i \in \mathcal{B}} V_{i}\left(\subseteq V^{(1)}\right)
$$

for $\mathcal{B} \subseteq\{1, \ldots, k\}$. I will consider the graph $\mathcal{G}$ on $\{1, \ldots, k\}$, whose edges are the sets of the form $\left\{i_{1}, i_{2}\right\}$ such that $V_{i_{1}} \cap V_{i_{2}} \neq \varnothing$. In view of c), it easily follows that $\mathcal{G}$ is a connected graph.

Next, I recall the definition of the renormalization operator $\Lambda_{r}$. For every $u \in \mathbb{R}^{V^{(0)}}$, every $E \in \mathcal{D}$ and every $r \in W$, let

$$
\Lambda_{r}(E)=\left(S_{r}^{\prime \prime}(E)\right)_{V^{(0)}}, \quad S_{r}^{\prime \prime}(E): \mathbb{R}^{V^{(1)}} \rightarrow \mathbb{R}, S_{r}^{\prime \prime}(E)(v):=\sum_{i=1}^{k} r_{i} E\left(v \circ \psi_{i}\right) .
$$

Note that $\Lambda_{r}$ maps $\mathcal{D}$ into itself and $\widetilde{\mathcal{D}}$ into itself. The problem discussed in the present paper is that of the existence of a G-eigenform in $\widetilde{\mathcal{D}}$, in other words, the existence of $E \in \widetilde{\mathcal{D}}$ such that $\Lambda_{r}(E)=\rho E$ for some $\rho>0$ and $r \in W$. Let

$$
\widehat{\Lambda}_{r}(q)=\widehat{\Lambda}(r, q):=\bar{I}^{-1} \circ \Lambda_{r} \circ \bar{I}(q) \quad \forall q \in \mathcal{Q} .
$$

Note that $\widehat{\Lambda}_{r}$ maps $\mathcal{Q}$ into itself and $\widetilde{\mathcal{Q}}$ into itself.

Lemma 3.1. The map $\widehat{\Lambda}$ from $W \times \mathcal{Q}$ to $\mathcal{Q}$ is continuous.

Proof. The map $q \mapsto \bar{I}(q)(u)$ from $\mathcal{Q}$ to $\mathbb{R}$ is continuous for every $u \in \mathbb{R}^{V^{(0)}}$. As a consequence, the map $(r, q) \mapsto S_{r}^{\prime \prime}(\bar{I}(q))(v)$ from $W \times \mathcal{Q}$ to $\mathbb{R}$ is continuous for every 
$v \in \mathbb{R}^{V^{(1)}}$. Thus, the map $(r, q) \mapsto \bar{I}^{-1}\left(S_{r}^{\prime \prime}(\bar{I}(q))\right)$ from $W \times \mathcal{Q}$ to $\mathcal{Q}\left(V^{(1)}\right)$ is continuous. By Lemma 2.4, the map $(r, q) \mapsto \Lambda_{r}(\bar{I}(q))(u)$ is continuous for every $u \in \mathbb{R}^{V^{(0)}}$. Hence, the map $(r, q) \mapsto \bar{I}^{-1}\left(\Lambda_{r}(\bar{I}(q))\right)=\widehat{\Lambda}(r, q)$ is continuous.

Given $A \subseteq\{1, \ldots, k\}, s \in] 0,+\infty\left[{ }^{A}\right.$ and $\left.\left.t \in\right] 0,+\infty\right]$, we define $\iota_{t}(s) \in W$ by

$$
\iota_{t}(s)_{i}= \begin{cases}s_{i} & \text { if } i \in A \\ t & \text { if } i \notin A\end{cases}
$$

More generally, we define

$$
S_{r, i, \mathcal{B}}^{\prime \prime}\left(E_{i}\right)(v)=\sum_{i \in \mathcal{B}} r_{i} E_{i}\left(v \circ \psi_{i}\right)
$$

when $E_{i} \in \widetilde{\mathcal{D}}$ for every $i \in \mathcal{B}$, when $\left.\varnothing \neq \mathcal{B} \subseteq\{1, \ldots, k\}, r \in\right] 0,+\infty\left[^{\mathcal{B}}, v \in \mathbb{R}^{V(\mathcal{B})}\right.$. Also, put

$$
S_{r, i, \mathcal{B}}^{\prime}\left(q_{i}\right)=S_{r, i, \mathcal{B}}^{\prime \prime}\left(\bar{I}\left(q_{i}\right)\right)
$$

when $q_{i} \in \widetilde{\mathcal{Q}}$ for every $i \in \mathcal{B}$, in other words, $q: \mathcal{B} \rightarrow \widetilde{\mathcal{Q}}$. We put shortly $S_{r, \mathcal{B}}^{\prime}\left(q_{i}\right)$ for $S_{r, i, \mathcal{B}}^{\prime}\left(q_{i}\right)$, and $S_{r, \mathcal{B}}^{\prime \prime}\left(E_{i}\right)=S_{r, i, \mathcal{B}}^{\prime \prime}\left(E_{i}\right)$. The following lemma is substantially well-known.

Lemma 3.2. We have $S_{r, \mathcal{B}}^{\prime}\left(q_{i}\right) \in \mathcal{D}(V(\mathcal{B}))$, and, if $\mathcal{B}$ is connected in $\mathcal{G}$, then $S_{r, \mathcal{B}}^{\prime}\left(q_{i}\right) \in$ $\widetilde{\mathcal{D}}(V(\mathcal{B}))$.

Put $S_{r, \mathcal{B}}(q)=\bar{I}^{-1}\left(S_{r, \mathcal{B}}^{\prime}(q)\right), S_{r, i, \mathcal{B}}\left(q_{i}\right)\left(=S_{r, \mathcal{B}}\left(q_{i}\right)\right)=\bar{I}^{-1}\left(S_{r, \mathcal{B}}^{\prime}\left(q_{i}\right)\right)$. We will shortly write $S_{r}^{\prime}$ for $S_{r, \mathcal{B}}^{\prime}, S_{r}^{\prime \prime}$ for $S_{r, \mathcal{B}}^{\prime \prime}$ and $S_{r}$ for $S_{r, \mathcal{B}}$ when $\mathcal{B}=\{1, \ldots, k\}$. Moreover, when $q_{i}=q$ for every $i \in \mathcal{B}$, we can put $S_{r, \mathcal{B}}^{\prime}\left(q_{i}\right)=S_{r, \mathcal{B}}^{\prime}(q)$ and similarly in the other cases. The following lemmas will be useful in the sequel.

Lemma 3.3. For every $q \in \widetilde{\mathcal{Q}}$ and $\left\{j_{1}, j_{2}\right\} \in J$ we have

$$
\widehat{R}\left(\widehat{\Lambda}_{r}(q)\right)_{\left\{P_{j_{1}}\right\},\left\{P_{j_{2}}\right\}}=\widehat{R}\left(S_{r}(q)\right)_{\left\{P_{j_{1}}\right\},\left\{P_{j_{2}}\right\}} .
$$

Proof. This is a particular case of Lemma 2.3.

Lemma 3.4. The map $(q, r) \mapsto \widehat{R}\left(S_{r, \mathcal{B}}(q)\right)_{\{A, B\}}$ is continuous from $\left.\mathcal{Q} \times\right] 0,+\infty\left[{ }^{\mathcal{B}} \rightarrow\right.$ ] $0,+\infty]$, for every $\varnothing \neq \mathcal{B} \subseteq\{1, \ldots, k\}$ and $A, B$ nptdss of $V(\mathcal{B})$.

Lemma 3.5. if $s: \mathcal{B} \rightarrow] 0,+\infty\left[\right.$, with $i \in \mathcal{B} \subseteq\{1, \ldots, k\}, q_{i} \in \mathcal{Q}$, then, if $A, B$ are nptdss of $V^{(0)}$, then $\widehat{R}\left(S_{s,\{i\}}\left(q_{i}\right)\right)_{\left\{\psi_{i}(A), \psi_{i}(B)\right\}}=\frac{1}{s_{i}} \widehat{R}\left(q_{i}\right)_{\{A, B\}}$.

Proof. For every $v \in V_{i}^{\mathbb{R}}$, we have $\bar{I}\left(S_{s,\{i\}}\left(q_{i}\right)\right)(v)=\bar{I}\left(s_{i} q_{i}\right)\left(v \circ \psi_{i}\right)$ and we conclude by the definition of $\widehat{R}$, identifying $V^{(0)}$ with $V_{i}$ via $\psi_{i}$. 
Remark 3.6. Suppose $\left.\varnothing \neq \mathcal{B}_{1} \subseteq \mathcal{B}_{2} \subseteq\{1, \ldots, k\}, r \in\right] 0,+\infty\left[\mathcal{B}_{2}, v \in \mathbb{R}^{V\left(\mathcal{B}_{2}\right)}\right.$. Then $S_{r, \mathcal{B}_{1}}^{\prime}(q)(v) \leq S_{r, \mathcal{B}_{2}}^{\prime}(q)(v)$. Therefore, if $A, B$ are nptdss of $V\left(\mathcal{B}_{1}\right)$, then $\widehat{R}\left(S_{r, \mathcal{B}_{1}}(q)\right)_{\{A, B\}} \geq$ $\widehat{R}\left(S_{r, \mathcal{B}_{2}}(q)\right)_{\{A, B\}}$.

We say that two subsets $\mathcal{B}$ and $\mathcal{B}^{\prime}$ of $\{1, \ldots, k\}$ are separated if $V(\mathcal{B}) \cap V\left(\mathcal{B}^{\prime}\right)=\varnothing$, in other words, if $V_{d} \cap V_{d^{\prime}}=\varnothing$ when $d \in \mathcal{B}, d^{\prime} \in \mathcal{B}^{\prime}$. Of course two separated subsets are disjoint, but the converse does not hold.

We will now prove two important lemmas (Lemma 3.7 and Lemma 3.9) concerning the relation between the effective conductivity with respect to a set $\mathcal{A}$ and with respect to a subset of $\mathcal{A}$. In particular, in part ii) of Lemma 3.7, roughly speaking, we prove that putting conductivity equal to infinity on a subset amounts to collapsing such a set. A similar consideration is given in [1]. Note that, for the uniform convergence, in place of the more natural condition of $(s, q)$ staying on compact sets, I here require a weaker and a bit technical condition, that however, apparently is necessary for the sequel (Lemma 8.4).

Lemma 3.7. Let $\mathcal{B}_{1}, \mathcal{B}_{2}, \mathcal{B}_{3}, \mathcal{B}_{4}$ be subsets of $\{1,2, \ldots, k\}$. Suppose $\mathcal{B}_{1}, \mathcal{B}_{2}, \mathcal{B}_{3}, \mathcal{B}_{4}$ are mutually disjoint and $\mathcal{B}_{1}, \mathcal{B}_{2}, \mathcal{B}_{4}$ are mutually separated. Put $\mathcal{A}:=\bigcup_{i=1}^{4} \mathcal{B}_{i}$. Suppose $M_{1}=$ $V\left(\mathcal{B}_{1}\right) \cap V\left(\mathcal{B}_{3}\right) \neq \varnothing, M_{2}=V\left(\mathcal{B}_{2}\right) \cap V\left(\mathcal{B}_{3}\right) \neq \varnothing$. Let $A_{1} \subseteq V\left(\mathcal{B}_{1}\right), A_{2} \subseteq V\left(\mathcal{B}_{2}\right)$ and suppose $A_{1} \cap M_{1}=\varnothing, A_{2} \cap M_{2}=\varnothing, A_{1}$ and $A_{2}$ nonempty. Then,

i) $\widehat{R}\left(S_{s^{\prime}, \mathcal{A}}(q)\right)_{\left\{A_{1}, A_{2}\right\}} \geq \widehat{R}\left(S_{s, \mathcal{B}_{1}}(q)\right)_{\left\{A_{1}, M_{1}\right\}}+\widehat{R}\left(S_{s, \mathcal{B}_{2}}(q)\right)_{\left\{A_{2}, M_{2}\right\}}$ if $\left.s^{\prime} \in\right] 0,+\infty[\mathcal{A}, s=$ $\left.s^{\prime}\right|_{\mathcal{B}_{1} \cup \mathcal{B}_{2} \cup \mathcal{B}_{4}}$

ii) If moreover, $\mathcal{B}_{3}$ is connected (in $\mathcal{G}$ ), then

$$
\widehat{R}\left(S_{\iota_{t}}(s), \mathcal{A}(q)\right)_{\left\{A_{1}, A_{2}\right\}} \underset{t \rightarrow+\infty}{\longrightarrow} \widehat{R}\left(S_{s, \mathcal{B}_{1}}(q)\right)_{\left\{A_{1}, M_{1}\right\}}+\widehat{R}\left(S_{s, \mathcal{B}_{2}}(q)\right)_{\left\{A_{2}, M_{2}\right\}}
$$

for $(s, q) \in] 0,+\infty\left[\mathcal{B}_{1} \cup \mathcal{B}_{2} \cup \mathcal{B}_{4} \times \widetilde{\mathcal{Q}}\right.$, and the convergence is uniform for $(s, q)$ in any subset $Z$ of $] 0,+\infty\left[\mathcal{B}_{1} \cup \mathcal{B}_{2} \cup \mathcal{B}_{4} \times \widetilde{\mathcal{Q}}\right.$ where $q \in K, s_{i} \leq A$ for every $i \in \mathcal{B}_{5}$ and the right-hand side of formula in ii) is $\leq B$, with $K$ compact subset of $\widetilde{\mathcal{Q}}$ and $\mathcal{B}_{5}$ is the set of $i \in \mathcal{B}_{1} \cup \mathcal{B}_{2} \cup \mathcal{B}_{4}$ such that $V_{i} \cap V\left(\mathcal{B}_{3}\right) \neq \varnothing$ and $A$ and $B$ are positive constants.

Proof. Let $s$ and $q$ be as above, and let $E=\bar{I}(q)$. Then,

$$
(1 / \widehat{R})\left(S_{s^{\prime}, \mathcal{A}}(q)\right)_{\left\{A_{1}, A_{2}\right\}}=\inf _{v \in \mathcal{L}_{V(\mathcal{A}) ; A_{1}, A_{2}}}\left(S_{s, \mathcal{B}_{1} \cup \mathcal{B}_{2} \cup \mathcal{B}_{4}}^{\prime}(q)(v)+S_{s^{\prime}, \mathcal{B}_{3}}^{\prime}(q)(v)\right)
$$

Let

$$
\left.\bar{S}=\inf _{v \in \bar{L}} S_{s^{\prime}, \mathcal{A}}^{\prime}(q)(v), \quad \bar{L}:=\left\{v \in \mathcal{L}_{V(\mathcal{A}) ; A_{1}, A_{2}}: v \text { constant on } V\left(\mathcal{B}_{3}\right)\right\} .\right)
$$

We clearly have $\bar{S}=\hat{S}$ where

$$
\hat{S}=\inf _{\tau \in \mathbb{R}}\left(\tau^{2}(1 / \widehat{R})\left(S_{s, \mathcal{B}_{1}}(q)\right)_{\left\{A_{1}, M_{1}\right\}}+(1-\tau)^{2}(1 / \widehat{R})\left(S_{s, \mathcal{B}_{2}}(q)\right)_{\left\{A_{2}, M_{2}\right\}}\right)
$$

In fact, if $v \in \bar{L}$, then $v=\tau$ on $V\left(\mathcal{B}_{3}\right)$ for some $\tau \in \mathbb{R}$. Hence, 


$$
\begin{gathered}
\left.S_{s, \mathcal{B}_{1} \cup \mathcal{B}_{2} \cup \mathcal{B}_{4}}^{\prime}(q)(v)+S_{s^{\prime}, \mathcal{B}_{3}}^{\prime}(q)(v)\right) \geq S_{s, \mathcal{B}_{1}}^{\prime}(q)(v)+S_{s, \mathcal{B}_{2}}^{\prime}(q)(v) \\
\geq \tau^{2}(1 / \widehat{R})\left(S_{s, \mathcal{B}_{1}}(q)\right)_{\left\{A_{1}, M_{1}\right\}}+(1-\tau)^{2}(1 / \widehat{R})\left(S_{s, \mathcal{B}_{2}}(q)\right)_{\left\{A_{2}, M_{2}\right\}} \geq \hat{S}
\end{gathered}
$$

by Lemma 2.9 , hence $\bar{S} \geq \hat{S}$. On the other hand, let $\tau$ attain the inf in the definition of $\hat{S}$. For such a $\tau$, by Lemma 2.9 again, there exist $v_{1} \in \mathbb{R}^{V\left(\mathcal{B}_{1}\right)}$ and $v_{2} \in \mathbb{R}^{V\left(\mathcal{B}_{2}\right)}$ such that

$$
\begin{gathered}
v_{1}=0 \text { on } A_{1}, \quad v_{1}=\tau \text { on } M_{1} \quad S_{s, \mathcal{B}_{1}}^{\prime}(q)\left(v_{1}\right)=\tau^{2}(1 / \widehat{R})\left(S_{s, \mathcal{B}_{1}}(q)\right)_{\left\{A_{1}, M_{1}\right\}} \\
v_{2}=1 \text { on } A_{2}, \quad v_{2}=\tau \text { on } M_{2} \quad S_{s, \mathcal{B}_{2}}^{\prime}(q)\left(v_{2}\right)=(1-\tau)^{2}(1 / \widehat{R})\left(S_{s, \mathcal{B}_{2}}(q)\right)_{\left\{A_{2}, M_{2}\right\}} .
\end{gathered}
$$

Now, defining $v \in \mathbb{R}^{V(\mathcal{A})}$ by $v(Q)=\left\{\begin{array}{ll}v_{1}(Q) & \text { if } Q \in V\left(\mathcal{B}_{1}\right), \\ v_{2}(Q) & \text { if } Q \in V\left(\mathcal{B}_{2}\right), \\ \tau & \text { otherwise }\end{array}\right.$ we easily see that $v \in \bar{L}$. Moreover, $S_{s^{\prime}, \mathcal{A}}^{\prime}(q)(v)=\hat{S}$ and formula $\hat{S}=\bar{S}$ is proved. By a standard argument, we thus obtain $\frac{1}{\bar{S}}=\widehat{R}\left(S_{s, \mathcal{B}_{1}}(q)\right)_{\left\{A_{1}, M_{1}\right\}}+\widehat{R}\left(S_{s, \mathcal{B}_{2}}(q)\right)_{\left\{A_{2}, M_{2}\right\}}$ and, by the definition of $\bar{S}$, i) is proved. In order to prove the convergence in ii), we will show that for every $\varepsilon>0$, for every $(s, q) \in Z$, for sufficiently large $t$, we have

$$
(1 / \widehat{R})\left(S_{\iota_{t}(s), \mathcal{A}}(q)\right)_{\left\{A_{1}, A_{2}\right\}} \geq \bar{S}-\varepsilon
$$

Take $\eta \in] 0,1\left[\right.$ so that if $v, v^{\prime}: V\left(\mathcal{B}_{5}\right) \rightarrow[-1,2]$ and $\max \left|v-v^{\prime}\right| \leq \eta$, then

$$
\left|S_{s, \mathcal{B}_{5}}^{\prime}(q)(v)-S_{s, \mathcal{B}_{5}}^{\prime}(q)\left(v^{\prime}\right)\right| \leq A \sum_{i \in \mathcal{B}_{5}}\left|\bar{I}(q)\left(v \circ \psi_{i}\right)-\bar{I}(q)\left(v^{\prime} \circ \psi_{i}\right)\right|<\varepsilon
$$

As $q \in K$ with $K$ compact, there exists $c_{1}>0$ such that $\bar{I}(q)(u) \geq c_{1} \bar{I}(\bar{q})(u)$ for every $u \in \mathbb{R}^{V^{(0)}}$, where $\bar{q}$ is a fixed element of $K$. By Lemmas 2.2 and 3.2, for every $v \in \mathbb{R}^{V\left(\mathcal{B}_{3}\right)}$, we thus have

$$
S_{1, \mathcal{B}_{3}}^{\prime}(q)(v) \geq c_{1} S_{1, \mathcal{B}_{3}}^{\prime}(\bar{q})(v) \geq c_{1} \bar{H}\left(\underset{V\left(\mathcal{B}_{3}\right)}{\operatorname{Osc}} v\right)^{2}
$$

for some positive constant $\bar{H}$. Hence, if $v_{t} \in \mathcal{L}_{V(\mathcal{A}) ; A_{1}, A_{2}}^{\prime}$ realizes the infimum in (3.1), as we have proved $\bar{S} \geq \hat{S}$, we obtain

$$
\bar{S} \geq S_{s, \mathcal{B}_{1} \cup \mathcal{B}_{2} \cup \mathcal{B}_{4}}^{\prime}(q)\left(v_{t}\right)+t S_{1, \mathcal{B}_{3}}^{\prime}(q)\left(v_{t}\right) \geq t c_{1} \bar{H}\left(\underset{V\left(\mathcal{B}_{3}\right)}{\operatorname{Osc}} v_{t}\right)^{2}
$$

thus, for every $\varepsilon>0$, for sufficiently large $t$, we have $\underset{V\left(\mathcal{B}_{3}\right)}{\operatorname{Osc}} v_{t} \leq \eta$. Therefore, there exists a constant $C$ such that $\left|v_{t}-C\right| \leq \eta$ on $V\left(\mathcal{B}_{3}\right)$. Let now $\bar{v}_{t}(Q)=\left\{\begin{array}{ll}v_{t}(Q) & \text { if } Q \notin V\left(\mathcal{B}_{3}\right) \\ C & \text { if } Q \in V\left(\mathcal{B}_{3}\right)\end{array}\right.$, $\bar{v}_{t} \in \mathbb{R}^{V(\mathcal{A})}$. Thus, $\max \left|\bar{v}_{t}-v_{t}\right| \leq \eta$, and by (3.2), putting $\mathcal{B}=\left(\mathcal{B}_{1} \cup \mathcal{B}_{2} \cup \mathcal{B}_{4}\right) \backslash \mathcal{B}_{5}$, we have $\left|S_{s, \mathcal{B}_{1} \cup \mathcal{B}_{2} \cup \mathcal{B}_{4}}^{\prime}(q)\left(v_{t}\right)-S_{s, \mathcal{B}_{1} \cup \mathcal{B}_{2} \cup \mathcal{B}_{4}}^{\prime}(q)\left(\bar{v}_{t}\right)\right|=\left|S_{s, \mathcal{B}_{5}}^{\prime}(q)\left(v_{t}\right)-S_{s, \mathcal{B}_{5}}^{\prime}(q)\left(\bar{v}_{t}\right)\right|<\varepsilon$ as $v_{t}, \bar{v}_{t}$ take value in $[-1,2]$. Thus, we have 


$$
\begin{gathered}
(1 / \widehat{R})\left(S_{\iota_{t}(s), \mathcal{A}}(q)\right)_{\left\{A_{1}, A_{2}\right\}}=S_{s, \mathcal{B}_{1} \cup \mathcal{B}_{2} \cup \mathcal{B}_{4}}^{\prime}(q)\left(v_{t}\right)+t S_{1, \mathcal{B}_{3}}^{\prime}(q)\left(v_{t}\right) \\
\geq S_{s, \mathcal{B}_{1} \cup \mathcal{B}_{2} \cup \mathcal{B}_{4}}^{\prime}(q)\left(\bar{v}_{t}\right)-\varepsilon=S_{\iota_{t}(s), \mathcal{A}}^{\prime}(q)\left(\bar{v}_{t}\right)-\varepsilon \geq \bar{S}-\varepsilon
\end{gathered}
$$

and we easily conclude.

Remark 3.8. Note that in Lemma $3.7 \mathcal{B}_{1}, \mathcal{B}_{2}$ and $\mathcal{B}_{3}$ are necessarily nonempty, while $\mathcal{B}_{4}$ could be empty.

Lemma 3.9. Suppose $\mathcal{A} \subseteq \mathcal{A}_{3,1}$ is the union of the mutually disjoint subsets $\mathcal{B}_{1}, \mathcal{B}_{2}$. Suppose $M=V\left(\mathcal{B}_{1}\right) \cap V\left(\mathcal{B}_{2}\right)$. Let $\varnothing \neq A_{i} \subseteq V\left(\mathcal{B}_{1}\right)$ with $A_{i} \cap M=\varnothing$ for $i=1,2$, $A_{1} \cap A_{2}=\varnothing$. Put $r_{i}=1$ for $i \in \mathcal{B}_{2}$. Then

$$
0 \leq(1 / \bar{R})\left(S_{r, \mathcal{A}}^{\prime}\left(q_{i}\right)\right)_{\left\{A_{1}, A_{2}\right\}}-(1 / \bar{R})\left(S_{r, \mathcal{B}_{1}}^{\prime}\left(q_{i}\right)\right)_{\left\{A_{1}, A_{2}\right\}} \leq k \max \left\{\left|q_{i}\right|: i \in \mathcal{B}_{2}\right\}
$$

Proof. Note that $A_{1}$ and $A_{2}$ are nonempty disjoint subsets both of $V(\mathcal{A})$ and of $V\left(\mathcal{B}_{1}\right)$. By Remark 3.6, $(1 / \bar{R})\left(S_{r, \mathcal{A}}^{\prime}\left(q_{i}\right)\right)_{\left\{A_{1}, A_{2}\right\}} \geq(1 / \bar{R})\left(S_{r, \mathcal{B}_{1}}^{\prime}\left(q_{i}\right)\right)_{\left\{A_{1}, A_{2}\right\}}$. On the other hand, for every $v \in \mathcal{L}_{V\left(\mathcal{B}_{1}\right) ; A_{1}, A_{2}}^{\prime}$, we extend $v$ to $w \in \mathcal{L}_{V(\mathcal{A}) ; A_{1}, A_{2}}^{\prime}$, putting $w=0$ on $V(\mathcal{A}) \backslash V\left(\mathcal{B}_{1}\right)$. Then

$$
\left(S_{r, \mathcal{A}}^{\prime}\left(q_{i}\right)\right)(w)-\left(S_{r, \mathcal{B}_{1}}^{\prime}\left(q_{i}\right)\right)(v)=\sum_{i \in \mathcal{B}_{2}} \bar{I}\left(q_{i}\right)\left(w \circ \psi_{i}\right) \leq k \max \left\{\left|q_{i}\right|: i \in \mathcal{B}_{2}\right\}
$$

as

$$
\bar{I}\left(q_{i}\right)\left(w \circ \psi_{i}\right)=\sum_{\left\{j_{1}, j_{2}\right\} \in J}\left(q_{i}\right)_{\left\{j_{1}, j_{2}\right\}}\left(w\left(\psi_{i}\left(P_{j_{1}}\right)\right)-w\left(\psi_{i}\left(P_{j_{2}}\right)\right)\right)^{2} \leq\left|q_{i}\right|
$$

for every $i \in \mathcal{B}_{2}$, and $\left(w\left(\psi_{i}\left(P_{j_{1}}\right)\right)-w\left(\psi_{i}\left(P_{j_{2}}\right)\right)\right)^{2} \leq 1$ as $0 \leq w \leq 1$, and $\#\left(\mathcal{B}_{2}\right) \leq k$. We conclude the proof, using the definition of $(1 / \widehat{R})$.

\section{Fractals with connected interior.}

In this Section, I will prove the existence of a $G$-eigenform for fractals with connected interior. We will say that the fractal triple is a connected interior fractal triple or shortly C.I.F.T if

i) $V_{j_{1}} \cap V_{j_{2}}=\varnothing$ if $j_{1}, j_{2}=1, \ldots, N, j_{1} \neq j_{2}$,

ii) the set $\{N+1, \ldots, k\}$ is connected in $\mathcal{G}$.

We will assume that i) and ii) hold in all of this section.

As a consequence, defining

$$
\widetilde{V}:=V(\{N+1, \ldots, k\})
$$

and, for $i=1, . ., N$, 


$$
\phi(i):=\left\{P_{j}: j=1, \ldots, N, \psi_{i}\left(P_{j}\right) \in \widetilde{V}\right\},
$$

we have that $\tilde{V} \neq \varnothing$, and $P_{i} \notin \phi(i) \neq \varnothing$, for every $i=1, \ldots, N$. Moreover,

$$
\psi_{h}(\phi(h))=V_{h} \cap \widetilde{V} \quad \forall h=1, \ldots, N .
$$

Note that this class of fractals generalizes both that considered in [1], and that considered in [8] when the eigenform is regular. I will prove that on these fractals there exists a G-eigenform. The idea of the proof is that the map $q \mapsto \widehat{\Lambda}\left(\iota_{t}(\sigma(q)), q\right)$ sends a suitable compact and convex neighborhood of $1_{J}$ into itself, for a suitable continuous map $\sigma$ : ] $0,+\infty\left[{ }^{J} \rightarrow\right] 0,+\infty\left[^{N}\right.$, that is we put weights on the vertex cells suitably, and in fact the weights are chosen so that the effective resistance between $P_{j}$ and $\phi(j)$ equals $\frac{1}{2}$ (multiplied by a constant $a$ ), so that, in view of Lemma 3.7, all the effective resistances between two different $P_{j}$ approximates 1 (times $a$ ), and $a$ is chosen so that the resistances $a_{J}$ come from the element $1_{J}$ of $\widetilde{\mathcal{Q}}$.

Lemma 4.1. Let $(s, q) \in] 0,+\infty\left[^{N} \times\right] 0,+\infty\left[{ }^{J}\right.$. Then, for every $\left\{j_{1}, j_{2}\right\} \in J$ we have

$$
\bar{R}\left(\widehat{\Lambda}\left(\iota_{t}(s), q\right)\right)_{\left\{j_{1}, j_{2}\right\}} \underset{t \rightarrow+\infty}{\longrightarrow} \frac{\widehat{R}(q)_{\left\{\left\{P_{j_{1}}\right\}, \phi\left(j_{1}\right)\right\}}}{s_{j_{1}}}+\frac{\widehat{R}(q)_{\left\{\left\{P_{j_{2}}\right\}, \phi\left(j_{2}\right)\right\}}}{s_{j_{2}}} .
$$

uniformly on the compact subsets of $] 0,+\infty\left[{ }^{N} \times\right] 0,+\infty\left[{ }^{J}\right.$.

Proof. By Lemma 3.3

$$
\bar{R}\left(\widehat{\Lambda}\left(\iota_{t}(s), q\right)\right)_{\left\{j_{1}, j_{2}\right\}}=\widehat{R}\left(\widehat{\Lambda}\left(\iota_{t}(s), q\right)\right)_{\left\{\left\{P_{j_{1}}\right\},\left\{P_{j_{2}}\right\}\right\}}=\widehat{R}\left(S_{\iota_{t}(s)}(q)\right)_{\left\{\left\{P_{j_{1}}\right\},\left\{P_{j_{2}}\right\}\right\}} .
$$

We now use Lemma 3.7 with $\mathcal{B}_{1}=\left\{j_{1}\right\}, \mathcal{B}_{2}=\left\{j_{2}\right\}, \mathcal{B}_{3}=\{N+1, \ldots, k\}, \mathcal{B}_{4}=\{1, \ldots, N\} \backslash$ $\left\{j_{1}, j_{2}\right\}$. In this way, $\mathcal{A}=\{1, \ldots, k\}$ and $V(\mathcal{A})=V^{(1)}, V\left(\mathcal{B}_{3}\right)=\widetilde{V}$. We have

$$
M_{1}=V_{j_{1}} \cap \tilde{V}=\psi_{j_{1}}\left(\phi\left(j_{1}\right)\right) \neq \varnothing, \quad M_{2}=V_{j_{2}} \cap \widetilde{V}=\psi_{j_{2}}\left(\phi\left(j_{2}\right)\right) \neq \varnothing .
$$

Moreover, put $A_{1}=\left\{P_{j_{1}}\right\} \subseteq V\left(\mathcal{B}_{1}\right), A_{2}=\left\{P_{j_{2}}\right\} \subseteq V\left(\mathcal{B}_{2}\right)$. Also, if $l=1$, 2, then $M_{l} \cap A_{l}=\varnothing$ since, if $Q \in M_{l} \cap A_{l}$, thus $Q=P_{j_{l}}=\psi_{j_{l}}\left(P_{j_{l}}\right) \in \psi_{j_{l}}\left(\phi\left(j_{l}\right)\right)$ and $P_{j_{l}} \in \phi\left(j_{l}\right)$, a contradiction. Next, $\mathcal{B}_{3}$ is connected by hypothesis ii), and, finally, $\mathcal{B}_{1}, \mathcal{B}_{2}$ and $\mathcal{B}_{4}$ are mutually separated by i). Thus, in view of Lemma 3.7 and Lemma 3.5, we obtain

$$
\begin{aligned}
\widehat{R}\left(S_{\iota_{t}(s)}(q)\right)_{\left\{\left\{P_{j_{1}}\right\},\left\{P_{j_{2}}\right\}\right\}} \underset{t \rightarrow+\infty}{\longrightarrow} \widehat{R}\left(S_{s,\left\{j_{1}\right\}}(q)\right)_{\left\{\left\{P_{j_{1}}\right\}, \psi_{j_{1}}\left(\phi\left(j_{1}\right)\right)\right\}}+\widehat{R}\left(S_{s,\left\{j_{2}\right\}}(q)\right)_{\left\{\left\{P_{j_{2}}\right\}, \psi_{j_{2}}\left(\phi\left(j_{2}\right)\right)\right\}} \\
=\frac{\widehat{R}(q)_{\left\{\left\{P_{j_{1}}\right\}, \phi\left(j_{1}\right)\right\}}}{s_{j_{1}}}+\frac{\widehat{R}(q)_{\left\{\left\{P_{j_{2}}\right\}, \phi\left(j_{2}\right)\right\}}}{s_{j_{2}}}
\end{aligned}
$$

uniformly on the compact subsets of $] 0,+\infty\left[{ }^{N} \times \widetilde{\mathcal{Q}}\right.$. 
Theorem 4.2 There exist $q \in] 0,+\infty\left[{ }^{J}, s \in\right] 0,+\infty\left[{ }^{N}\right.$ and $t>0$ such that $\widehat{\Lambda}\left(\iota_{t}(s), q\right)=q$. Proof. By a symmetry argument, $\bar{R}\left(1_{J}\right)=a_{J}$ for some $a>0$. By Lemma 2.8 ii), there exists $\left.\delta_{1} \in\right] 0, a[$ such that

$$
\left[a-\delta_{1}, a+\delta_{1}\right]^{J} \subseteq \bar{R}(] 0,+\infty\left[^{J}\right) .
$$

On the other hand, it is well known that $\widehat{\Lambda}(r, q) \in] 0,+\infty\left[{ }^{J}\right.$ for every $\left.r \in W, q \in\right] 0,+\infty\left[^{J}\right.$. Therefore, by Lemma $2.8 \mathrm{ii}$ ) again, given $\eta \in] 0,1[$ there exists $\delta \in] 0, \delta_{1}$ [ such that, if $q \in] 0,+\infty\left[{ }^{J}\right.$ and $\bar{R}(q) \in[a-\delta, a+\delta]^{J}$, then $q \in[1-\eta, 1+\eta]^{J}$. For $q \in[1-\eta, 1+\eta]^{J}$, define $\sigma(q)_{j}:=\frac{2 \widehat{R}(q)_{\left\{\left\{P_{j}\right\}, \phi(j)\right\}}}{a}$ for every $j=1, \ldots, N$. Then, by Lemma 4.1, for sufficiently large $t$, independent of $q$,

$$
\bar{R}\left(\widehat{\Lambda}\left(\iota_{t}(\sigma(q)), q\right)\right)_{\left\{j_{1}, j_{2}\right\}} \in[a-\delta, a+\delta] \quad \forall\left\{j_{1}, j_{2}\right\} \in J,
$$

hence, $\widehat{\Lambda}\left(\iota_{t}(\sigma(q)), q\right) \in[1-\eta, 1+\eta]^{J}$. Moreover, $\left.\sigma:[1-\eta, 1+\eta]^{J} \rightarrow\right] 0,+\infty\left[^{N}\right.$ is continuous by Lemma 2.4. Thus, for such $t$, the map $q \mapsto \widehat{\Lambda}\left(\iota_{t}(\sigma(q)), q\right)$ sends continuously $[1-\eta, 1+\eta]^{J}$ into itself, hence it has a fixed point.

Corollary 4.3 There exist $E \in \widetilde{\mathcal{D}}$ and $r \in W$ such that $\Lambda_{r}(E)=E$.

Proof. It suffices to take $E=\bar{I}(q)$, where $q$ is as in Theorem 4.2 and $r=\iota_{t}(s)$.

Remark 4.4 It follows that the eigenform obtained as above is regular (this means that $r_{i}>\rho$ for every $\left.i=1, \ldots, k\right)$ as, by a known and standard argument, this is always true when $r_{i} \leq r_{i^{\prime}}$ when $i \leq N, i^{\prime}>N$. See for example [4] for the notion of regular eigenform.

\section{The structure of $V^{(n)}$ on General Fractals}

A self-similar fractal set in $\mathbb{R}^{\nu}$ is defined by a set of contracting similarities $\Psi:=$ $\left\{\psi_{1}, \ldots, \psi_{k}\right\}, k \geq 2$ from $\mathbb{R}^{\nu}$ into itself, that is there exists $\left.\alpha_{i} \in\right] 0,1\left[\right.$ such that $\| \psi_{i}\left(x_{1}\right)-$ $\psi_{i}\left(x_{2}\right)\left\|=\alpha_{i}\right\| x_{1}-x_{2} \|$ for every $x_{1}, x_{2} \in \mathbb{R}^{\nu}$. The fractal is defined as the unique nonempty compact set $K$ such that $K=\bigcup_{i=1}^{k} \psi_{i}(K)$. For example, the Sierpinski Gasket is defined by the set of similarities $\left\{\psi_{i}: i=1,2,3\right\}$ where $\psi_{i}(x)=\frac{1}{2}\left(x+P_{i}\right)$ and $P_{i}$ are the vertices of an equilateral triangle. Under some condition, we can associate a fractal triple by taking $V^{(0)}$ to be a subset with $N \geq 2$ elements of the set of the fixed points of $\psi_{i}$. Note that we can also define the same fractal $K$ replacing the set $\left\{\psi_{i}: i=1, \ldots k\right\}$ by the set $\Psi^{n}:=\left\{\psi_{i_{1}} \circ \cdots \circ \psi_{i_{n}}: i_{1}, \ldots, i_{n}=1, \ldots, k\right\}$. For the more general setting of the P.C.F. self-similar sets see [4]. Conversely, there exists a standard way to associate to a given fractal triple a self-similar fractal $K$, and also an $n$-fractal triple corresponding to the previously defined set $\Psi^{n}$ of one-to-one maps and generating the same fractal $K$. 
Namely, given a fractal triple $\mathcal{F}:=\left(V^{(0)}, V^{(1)}, \Psi\right), \Psi=\left\{\psi_{1}, \ldots, \psi_{k}\right\}$, we can define a related fractal, $\mathcal{F}$ in the following way: Let

$$
\widetilde{K}=\{1, \ldots, k\}^{\mathbb{N} \backslash\{0\}} .
$$

We denote an element of $\widetilde{K}$ by $\left(i_{1}, i_{2}, \ldots\right)$ with $i_{l}=1, \ldots, k$ for $l=1,2, \ldots$. The fractal set $K$ will be defined as $\widetilde{K}$ quotiented by the equivalence relation $\mathcal{R}$ that identifies two different elements $\left(i_{1}, i_{2}, \ldots\right)$ and $\left(i_{1}^{\prime}, i_{2}^{\prime}, \ldots\right)$ if there exists $\zeta=1,2, \ldots$ such that

$$
\left\{\begin{array}{l}
i_{s}=i_{s}^{\prime} \forall s<\zeta \\
i_{\zeta} \neq i_{\zeta}^{\prime}, i_{\zeta+1}, i_{\zeta+1}^{\prime} \leq N, \psi_{i_{\zeta}}\left(P_{i_{\zeta+1}}\right)=\psi_{i_{\zeta}^{\prime}}\left(P_{i_{\zeta+1}^{\prime}}\right), \\
i_{s}=i_{\zeta+1}, i_{s}^{\prime}=i_{\zeta+1}^{\prime} \forall s>\zeta .
\end{array}\right.
$$

Such a number $\zeta$ is clearly unique. It is simple to verify that $\mathcal{R}$ is in fact an equivalence relation and, in view of $b$ ) of the definition of a fractal triple, that the equivalence class of $(j, j, j, \ldots), j \leq N$, is a singleton. I will denote the equivalence class of $\left(i_{1}, i_{2}, \ldots\right)$ by $\left[i_{1}, i_{2}, \ldots\right]$. We define $\psi_{i}$ on $K$ as

$$
\psi_{i}\left(\left[i_{1}, i_{2} \ldots\right]\right)=\left[i, i_{1}, i_{2}, \ldots\right] \quad \forall i=1, \ldots, k
$$

In this way, every $\psi_{i}$ is a one-to-one map from $K$ into itself, and we put $\Psi:=\left\{\psi_{i}\right.$ : $i=1, \ldots, k\}$. When $j, j^{\prime} \leq N$, then $\psi_{j}([j, j, j, \ldots .])=.[j, j, \ldots]$, and also, $\psi_{i}([j, j, j, \ldots .])=$. $\psi_{i^{\prime}}\left(\left[j^{\prime}, j^{\prime}, j^{\prime}, \ldots.\right]\right)$ amounts to $\psi_{i}\left(P_{j}\right)=\psi_{i^{\prime}}\left(P_{j^{\prime}}\right)$, thus we put $P_{j}:=[j, j, j, \ldots .$.$] if j=$ $1, \ldots, N$, and $V=V^{(0)}=\left\{P_{j}, j=1, \ldots, N\right\}$. In this way, $K$ is an extension of the previously defined $V^{(0)}$, and $\psi_{i}$ extends on $K$ the previously defined $\psi_{i}$ on $V^{(0)}$. We put

$$
\psi_{i_{1}, \ldots, i_{n}}:=\psi_{1_{1}} \circ \cdots \circ \psi_{i_{n}}, \quad A_{i_{1}, \ldots, i_{n}}=\psi_{i_{1}, \ldots, i_{n}}(A) \quad \forall A \subseteq K .
$$

We also put

$$
V^{(n)}:=\bigcup_{i_{1}, i_{2}, \ldots, i_{n}=1}^{k} V_{i_{1}, \ldots, i_{n}}, \quad V^{(\infty)}=\bigcup_{n=1}^{\infty} V^{(n)} .
$$

The sets $V_{i_{1}, \ldots, i_{n}}$ are called $n$-cells. The following Lemma can be easily verified.

\section{Lemma 5.1.}

i) If $\psi_{i_{1}, \ldots, i_{n}}(Q)=P_{j}$, with $Q \in K, h, j=1, \ldots, N$, then $Q=P_{j}$ and $i_{1}=i_{2}=\ldots=i_{n}=j$. ii) If $\left(i_{1}, \ldots, i_{n}\right) \neq\left(i_{1}^{\prime}, \ldots, i_{n}^{\prime}\right)$, then

$$
K_{i_{1}, \ldots, i_{n}} \cap K_{i_{1}^{\prime}, \ldots, i_{n}^{\prime}}=V_{i_{1}, \ldots, i_{n}} \cap V_{i_{1}^{\prime}, \ldots, i_{n}^{\prime}} .
$$

(nesting axiom)

iii) If $\left(i_{1}, \ldots, i_{n}\right) \neq\left(i_{1}^{\prime}, \ldots, i_{n}^{\prime}\right)$ and $\psi_{\left(i_{1}, \ldots, i_{n}\right)}(Q)=\psi_{\left(i_{1}^{\prime}, \ldots, i_{n}^{\prime}\right)}\left(Q^{\prime}\right)$ with $Q, Q^{\prime} \in K$, then $Q, Q^{\prime} \in V^{(0)}$.

Corollary 5.2. If $i_{1}, \ldots, i_{n}, i_{1}^{\prime}, \ldots, i_{n}^{\prime}=1, \ldots, k, j, j^{\prime}=1, \ldots, N$, and 


$$
\psi_{i_{1}, \ldots, i_{n}}\left(P_{j}\right)=\psi_{i_{1}^{\prime}, \ldots, i_{n}^{\prime}}\left(P_{j^{\prime}}\right), \quad\left(i_{1}, \ldots, i_{n}\right) \neq\left(i_{1}^{\prime}, \ldots, i_{n}^{\prime}\right)
$$

then there exists $\bar{s}=1, \ldots, n$ such that $i_{s}=i_{s}^{\prime}$ for $1 \leq s<\bar{s}, i_{s}=j$ and $i_{s}^{\prime}=j^{\prime}$ for $\bar{s}<s \leq n$.

Proof. Let $\bar{s}$ be the smallest $s$ such that $i_{s} \neq i_{s}^{\prime}$. Then

$$
Q:=\psi_{i_{1}, \ldots, i_{n}}\left(P_{j}\right)=\psi_{i_{1}, \ldots, i_{\bar{s}}}\left(\psi_{i_{\bar{s}+1}, \ldots, i_{n}}\left(P_{j}\right)\right)=\psi_{i_{1}^{\prime}, \ldots, i_{\bar{s}}^{\prime}}\left(\psi_{i_{\bar{s}+1}^{\prime}, \ldots, i_{n}^{\prime}}\left(P_{j^{\prime}}\right)\right)
$$

thus, by Lemma $5.1 \mathrm{iii}), \psi_{i_{\bar{s}+1}, \ldots, i_{n}}\left(P_{j}\right) \in V^{(0)}$, hence for every $s>\bar{s}$ we have $i_{s}=j$ and by a similar argument $i_{s}^{\prime}=j^{\prime}$.

We equip $K$ with a topology taking as a basis of neighbourhoods of $Q \in K$ the set

$$
U_{n}=\bigcup_{Q \in K_{i_{1}, \ldots, i_{n}}} K_{i_{1}, \ldots, i_{n}}, \quad n=1,2, \ldots
$$

Given the fractal triple $\mathcal{F}$, we also define a related $n$-fractal triple $\mathcal{F}^{n}$ by

$$
\mathcal{F}^{n}:=\left(V^{(0)}, V^{(n)}, \Psi^{n}\right), \quad \Psi^{n}:=\left\{\psi_{i_{1}, \ldots, i_{n}}: i_{1}, \ldots, i_{n}=1, \ldots, k\right\} .
$$

Here, the map $\psi \in \Psi_{n}$ satisfying $\psi\left(P_{j}\right)=P_{j}$ is $\psi_{(j, j, \ldots, j)}$ for every $j \leq N$. It can be easily proved that in fact $\mathcal{F}^{n}$ is a fractal triple. Indeed, b) in the definition of a fractal triple follows from Lemma $5.1 \mathrm{i}$ ), and c) can be proved by induction on $n$. It can be easily verified that the fractal generated by $\mathcal{F}_{n}$ is the same as that generated by $\mathcal{F}$ and with the same topology. The rest of the present paper is devoted to prove

(Main Theorem) For every fractal triple, there exist $n \geq 1$ and a G-eigenform on $\mathcal{F}_{n}$.

This provides a self-similar energy on the fractal generated by the fractal structure. Given a fractal triple $\mathcal{F}:=\left(V^{(0)}, V^{(1)}, \Psi\right)$, we put

$$
\mathcal{A}_{1}(\mathcal{F})=\{1, \ldots, N\}, \quad \mathcal{A}_{2}(\mathcal{F})=\{N+1, \ldots, k\}, \quad \mathcal{A}_{3}(\mathcal{F})=\mathcal{A}_{1}(\mathcal{F}) \cup \mathcal{A}_{2}(\mathcal{F})=\{1, \ldots, k\}
$$

The rest of this section is devoted to the main properties of the $n$-fractal triples $\mathcal{F}_{n}$. Of course, $\mathcal{A}_{2}(\mathcal{F})$ could be empty. In some sense $\mathcal{A}_{3}$ is the set of the indices of the maps in $\Psi$, and $\mathcal{A}_{1}$ the set of the indices of the maps that fix an element of $V^{(0)}$. We say that a subset $\mathcal{A}(\mathcal{F})$ of $\mathcal{A}_{3}(\mathcal{F})$ is connected if it is connected in $\mathcal{G}$. We put $\mathcal{A}_{i, n}(\mathcal{F})=\mathcal{A}_{i}\left(\mathcal{F}_{n}\right)$. We write shortly $\mathcal{A}_{i}$ for $\mathcal{A}_{i}(\mathcal{F})$ and $\mathcal{A}_{i, n}$ for $\mathcal{A}_{i, n}(\mathcal{F})$ when $\mathcal{F}$ is clear from the context. We identify $\mathcal{A}_{3, n}$ with the set $\left\{\left(i_{1}, \ldots, i_{n}\right): i_{1}, \ldots, i_{n}=1, \ldots, k\right\}$. Of course, by this identification we have $\mathcal{A}_{1, n}=\left\{j_{(n)}: j=1, \ldots, N\right\}$. We put $\left.W_{n}=\right] 0,+\infty\left[\mathcal{A}_{3, n}\right.$.

Note that, if $n>1$, then $\mathcal{A}_{2, n}$ is nonempty in any case as it contains every object of the form $\left(j_{1}, j_{2}, \ldots\right)$ with $j_{1} \neq j_{2}$.

In the following, we will denote by $\varpi$ or ${ }_{n} \varpi$ or similar the elements $\left(i_{1}, \ldots, i_{n}\right)$ of $\mathcal{A}_{3, n}$. In such a case we put $V_{\varpi}:=V_{i_{1}, \ldots, i_{n}}$, and, if $\mathcal{A}$ is a subset of $\mathcal{A}_{3, n}$, 


$$
V(\mathcal{A})=\bigcup_{\varpi \in \mathcal{A}} V_{\varpi}, \quad K(\mathcal{A})=\bigcup_{\varpi \in \mathcal{A}} K_{\varpi}
$$

We call such sets respectively $V$-copy of $A$, and $K$-copy of $A$. Note that

$$
V\left(\mathcal{A}_{3, n}\right)=\bigcup_{i_{1}, \ldots, i_{n}=1}^{k} V_{i_{1}, \ldots, i_{k}}=V^{(n)} .
$$

By the previous identification, $\mathcal{G}\left(\mathcal{F}_{n}\right)=\mathcal{G}_{n}$ is the graph on $\mathcal{A}_{3, n}$ where the edges are the pairs $\left\{\varpi, \varpi^{\prime}\right\}$ such that $\varpi \neq \varpi^{\prime}$ and $V_{\varpi} \cap V_{\varpi^{\prime}} \neq \varnothing$. In the following, the notions concerning graphs on $\mathcal{A}_{3, n}$ will be always referred to $\mathcal{G}_{n}$, for example path will mean path in $\mathcal{G}_{n}$, connected will mean connected in $\mathcal{G}_{n}$ and so on. Let $\Pi_{n, \bar{n}}: \mathcal{A}_{3, n} \rightarrow \mathcal{A}_{3, \bar{n}}$ be defined by $\Pi_{n, \bar{n}}\left(\left(i_{1}, \ldots, i_{n}\right)\right)=\left(i_{1}, \ldots, i_{\bar{n}}\right)$, when $\bar{n} \leq n$, and we define naturally $\Pi_{n, \bar{n}}(C)=$ $\left\{\Pi_{n, \bar{n}}(\varpi): \varpi \in C\right\}$ for every $C \subseteq \mathcal{A}_{3, n}$. Of course, for every $C \subseteq \mathcal{A}_{3, \bar{n}}$ we have

$$
\Pi_{n, \bar{n}}^{-1}(C):=C \times \mathcal{A}_{3, n-\bar{n}} \quad(\neq \varnothing \quad \text { if } C \neq \varnothing) .
$$

Note that

$$
\Pi_{n, \bar{n}}^{-1}\left(\mathcal{A}_{2, \bar{n}}\right) \subseteq \mathcal{A}_{2, n} .
$$

In the following Lemmas 5.3, 5.4, 5.5, we will discuss the relationships between $V$-copy or $K$-copy of a set and of its image or preimage with respect to the projection $\Pi_{n, \bar{n}}$. The proof essentially depends on Lemma 5.1.

Lemma 5.3. Suppose $n \geq \bar{n}, \varpi \in \mathcal{A}_{3, n}$. Then,

i) $V_{\varpi} \subseteq K_{\Pi_{n, \bar{n}}(\varpi)} \quad \forall n \geq \bar{n}, \quad \forall \varpi \in \mathcal{A}_{3, n}$

ii) If moreover, $\varpi^{\prime} \in \mathcal{A}_{3, n}$ and $\Pi_{n, \bar{n}}(\varpi) \neq \Pi_{n, \bar{n}}\left(\varpi^{\prime}\right)$, then $V_{\varpi} \cap V_{\varpi^{\prime}} \subseteq V_{\Pi_{n, \bar{n}}(\varpi)} \cap V_{\Pi_{n, \bar{n}}\left(\varpi^{\prime}\right)}$.

Proof. In fact, if $Q \in V_{\varpi}, \varpi=\left(i_{1}, \ldots, i_{n}\right)$, then, for some $P_{j} \in V^{(0)}$, we have

$$
Q=\psi_{i_{1}, \ldots, i_{\bar{n}}}\left(\psi_{i_{\bar{n}+1}, \ldots, i_{n}}\left(P_{j}\right)\right)=\psi_{\Pi_{n, \bar{n}}(\varpi)}\left(\psi_{i_{\bar{n}+1}, \ldots, i_{n}}\left(P_{j}\right)\right), \quad \psi_{i_{\bar{n}+1}, \ldots, i_{n}}\left(P_{j}\right) \in K .
$$

and i) is proved. To prove ii), note in view of i) and Lemma 5.1 ii),

$$
V_{\varpi} \cap V_{\varpi^{\prime}} \subseteq K_{\Pi_{n, \bar{n}}(\varpi)} \cap K_{\Pi_{n, \bar{n}}\left(\varpi^{\prime}\right)}=V_{\Pi_{n, \bar{n}}(\varpi)} \cap V_{\Pi_{n, \bar{n}}\left(\varpi^{\prime}\right)} \cdot
$$

Lemma 5.4. Given $\bar{n}$ and $B, B^{\prime} \subseteq \mathcal{A}_{3, \bar{n}}, B \cap B^{\prime}=\varnothing$, then for every $n \geq \bar{n}$ we have i) $K(B) \supseteq V\left(\Pi_{n, \bar{n}}^{-1}(B)\right) \supseteq V(B)$,

ii) $V\left(\Pi_{n, \bar{n}}^{-1}(B)\right) \cap V\left(\Pi_{n, \bar{n}}^{-1}\left(B^{\prime}\right)\right)=V(B) \cap V\left(B^{\prime}\right)$,

iii) If $\varpi, \varpi^{\prime} \in \mathcal{A}_{3, \bar{n}}, \varpi \neq \varpi^{\prime}$, then $V\left(\Pi_{n, \bar{n}}^{-1}(\{\varpi\})\right) \cap V\left(\Pi_{n, \bar{n}}^{-1}\left(\left\{\varpi^{\prime}\right\}\right)\right)=V_{\varpi} \cap V_{\varpi^{\prime}}$

Proof. i) The first inclusion in i) follows from Lemma 5.3 i). To prove the second, note that, if $P \in V(B)$, then $P=\psi_{\varpi}\left(P_{j}\right)$ for some $j=1, \ldots, N$ and for some $\varpi \in B$. Therefore, $P=\psi_{\left(\varpi, j_{(n-\bar{n})}\right)}\left(P_{j}\right) \in V\left(\Pi_{n, \bar{n}}^{-1}(B)\right)$. ii) Let $P \in V\left(\Pi_{n, \bar{n}}^{-1}(B)\right) \cap V\left(\Pi_{n, \bar{n}}^{-1}\left(B^{\prime}\right)\right)$. Then 
$P \in K_{\varpi} \cap K_{\varpi^{\prime}}$, with $\varpi \in B, \varpi^{\prime} \in B^{\prime}$. Consequently, by the nesting axiom, $P \in$ $V_{\varpi} \cap V_{\varpi^{\prime}} \subseteq V(B) \cap V\left(B^{\prime}\right)$. In conclusion, we have proved the inclusion $\subseteq$ in ii), and the opposite inclusion is trivial. Finally, iii) is a particular case of ii).

\section{Lemma 5.5.}

i) If $\bar{n}>1, n \geq \bar{n}, n^{\prime} \geq \bar{n}-1, \varpi \in \mathcal{A}_{3, n}, \varpi^{\prime} \in \mathcal{A}_{3, n^{\prime}}$ and $\varpi \in \Pi_{n, \bar{n}}^{-1}\left(j_{(\bar{n})}\right)$, $\varpi^{\prime} \notin$ $\Pi_{n^{\prime}, \bar{n}-1}^{-1}\left(j_{(\bar{n}-1)}\right)$, then $V_{\varpi} \cap V_{\varpi^{\prime}}=\varnothing$.

ii) If $n>1$, we have $V_{j_{(n)}} \cap V_{i_{1}, i_{2}, \ldots, i_{n}}=\varnothing$ if $\left(i_{1}, \ldots, i_{n-1}\right) \neq j_{(n-1)}$. In particular, $V_{j_{(n)}} \cap$ $V_{j_{(n)}^{\prime}}=\varnothing$ for every $j, j^{\prime}=1, \ldots, N, j \neq j^{\prime}$, thus $\mathcal{F}_{n}$ satisfies i) in the definition of a connected interior fractal triple.

Proof. Suppose $Q \in V_{\varpi} \cap V_{\varpi^{\prime}}$ and $\varpi^{\prime}=\left(i_{1}, \ldots, i_{n^{\prime}}\right)$ with $\left(i_{1}, \ldots, i_{\bar{n}-1}\right) \neq j_{(\bar{n}-1)}$. Then, by Lemma $5.4 \mathrm{i}$ ), there exist $P, P^{\prime} \in K$ such that $Q=\psi_{j_{(\bar{n})}}(P)=\psi_{i_{1}, \ldots, i_{\bar{n}}-1}\left(P^{\prime}\right)$, hence

$$
\psi_{j_{(\bar{n}-1)}}\left(\psi_{j}(P)\right)=\psi_{i_{1}, \ldots, i_{\bar{n}-1}}\left(P^{\prime}\right) \text {. }
$$

By Lemma $5.1 \mathrm{iii}$ ), we deduce $\psi_{j}(P) \in V^{(0)}$ and by Lemma $\left.5.1 \mathrm{i}\right), P=P_{j}$, thus $\psi_{j}(P)=P_{j}$, thus $\psi_{i_{1}, \ldots, i_{\bar{n}-1}}\left(P^{\prime}\right)=\psi_{j_{(\bar{n}-1)}}\left(P_{j}\right)=P_{j}$, and as $\left(i_{1}, \ldots, i_{\bar{n}-1}\right) \neq j_{(\bar{n}-1)}$, this contradicts Lemma $5.1 \mathrm{i}$ ), thus i) is proved, and ii) is a particular case of $\mathrm{i}$ ).

In the following Lemmas 5.6, 5.7, 5.8, I will discuss relationships between separation of two sets, or connectedness of a set, and the corresponding properties of the images or preimages of the sets via the map $\Pi_{n, \bar{n}}$. In particular, in Lemma 5.8 gives a simple and useful criterion for connecting two points in a set.

Lemma 5.6. Given two subsets $\mathcal{B}_{1}$ and $\mathcal{B}_{2}$ of $\mathcal{A}_{3, \bar{n}}$, then

i) $\mathcal{B}_{1}$ and $\mathcal{B}_{2}$ are disjoint if and only if so are $\Pi_{n, \bar{n}}^{-1}\left(\mathcal{B}_{1}\right), \Pi_{n, \bar{n}}^{-1}\left(\mathcal{B}_{2}\right)$

ii) $\mathcal{B}_{1}$ and $\mathcal{B}_{2}$ are separated if and only if so are $\Pi_{n, \bar{n}}^{-1}\left(\mathcal{B}_{1}\right)$ and $\Pi_{n, \bar{n}}^{-1}\left(\mathcal{B}_{2}\right)$.

Proof. Point i) immediately follows from (5.1), and ii) holds by Lemma 5.4 ii) provided $\mathcal{B}_{1}$ and $\mathcal{B}_{2}$ are disjoint. But if they are not disjoint, $\mathcal{B}_{1}$ and $\mathcal{B}_{2}$ are not separated, as well as $\Pi_{n, \bar{n}}^{-1}\left(\mathcal{B}_{1}\right)$ and $\Pi_{n, \bar{n}}^{-1}\left(\mathcal{B}_{2}\right)$.

\section{Lemma 5.7.}

i) A subset $\mathcal{A}$ of $\mathcal{A}_{3, \bar{n}}$ is connected if and only if so is $\Pi_{n, \bar{n}}^{-1}(\mathcal{A})$ for every $n \geq \bar{n}$

ii) if $\mathcal{A} \subseteq \mathcal{A}_{3, n}$ is connected, then so is $\Pi_{n, \bar{n}}(\mathcal{A})$.

Proof. To prove i) first of all, note that $\Pi_{n, \bar{n}}^{-1}(\varpi)$ is connected for every $\varpi \in \mathcal{A}_{3, \bar{n}}$, as it is isomorphic to $\mathcal{A}_{3, n-n_{2}}$. Now, if $\mathcal{A} \subseteq \mathcal{A}_{3, \bar{n}}$ is connected, let $\varpi, \varpi^{\prime} \in \Pi_{n, \bar{n}}^{-1}(\mathcal{A})$, so that $\Pi_{n, \bar{n}}(\varpi)={ }_{n} \varpi \in \mathcal{A}, \Pi_{n, \bar{n}}\left(\varpi^{\prime}\right)={ }_{n} \varpi^{\prime} \in \mathcal{A}$. Let $\left({ }_{n} \varpi_{0}, \ldots, \bar{n}_{n} \varpi_{m}\right)$ be a path in $\mathcal{A}$ connecting $\bar{n} \varpi$ to $\bar{n}^{\prime}$. By Lemma 5.4 iii), as $V_{\bar{n} \varpi_{i-1}} \cap V_{\bar{n} \varpi_{i}} \neq \varnothing$, there exist $\varpi_{i}^{-} \in \Pi_{n, \bar{n}}^{-1}\left(\bar{n} \varpi_{i}\right)$, $\varpi_{i-1}^{+} \in \Pi_{n, \bar{n}}^{-1}\left(\bar{n}_{i-1}\right)$ so that $V_{\varpi_{i-1}^{+}} \cap V_{\varpi_{i}^{-}} \neq \varnothing$. It follows that $\varpi_{i-1}^{+} \sim \varpi_{i}^{-}$or $\varpi_{i-1}^{+}=\varpi_{i}^{-}$, hence $\varpi$ is connected to $\varpi^{\prime}$ in $\Pi_{n, \bar{n}}^{-1}(\mathcal{A})$.

To prove the converse, it suffices to note that, if $\left(\varpi_{0}, \ldots, \varpi_{m}\right)$ is a path in $\mathcal{B} \subseteq \mathcal{A}_{3, n}$ connecting $\varpi$ to $\varpi^{\prime}$, then, by Lemma $\left.5.3 \mathrm{ii}\right)$, the image $\left(\Pi_{n, \bar{n}}\left(\varpi_{0}\right), \ldots, \Pi_{n, \bar{n}}\left(\varpi_{m}\right)\right)$ is a 
weak path in $\Pi_{n, \bar{n}}(\mathcal{B})$ connecting $\Pi_{n, \bar{n}}(\varpi)$ to $\Pi_{n, \bar{n}}\left(\varpi^{\prime}\right)$ and i) is proved. In a similar way we can prove ii).

Lemma 5.8. If $n \geq \bar{n}, \bar{n} \varpi, \bar{n} \varpi^{\prime} \in \mathcal{A}_{3, \bar{n}}, \mathcal{B}_{1}, \mathcal{B}_{2} \subseteq \mathcal{A}_{3, \bar{n}}$ and $\bar{n} \varpi$ is connected to $\bar{n} \varpi^{\prime}$ in $\mathcal{B}_{1} \cup \mathcal{B}_{2}, \bar{n} \varpi \in \mathcal{B}_{1} \backslash \mathcal{B}_{2}, \bar{n} \varpi^{\prime} \in \mathcal{B}_{2}$, then every $\varpi \in \Pi_{n, \bar{n}}^{-1}(\bar{n} \varpi)$ is connected to some $\varpi^{\prime} \in \Pi_{n, \bar{n}}^{-1}\left(\mathcal{B}_{2}\right)$ in $\Pi_{n, \bar{n}}^{-1}\left(\mathcal{B}_{1}\right)$.

Proof. Let $A$ be a path connecting ${ }_{n} \varpi$ to ${ }_{n} \varpi^{\prime}$ in $\mathcal{B}_{1} \cup \mathcal{B}_{2}$, thus $A \subseteq \mathcal{B}_{1} \cup \mathcal{B}_{2}$. Then $B:=\Pi_{n, \bar{n}}^{-1}(A)$ is connected, and if $\varpi^{\prime \prime}$ is any element in $\Pi_{n, \bar{n}}^{-1}\left(\bar{n} \varpi^{\prime}\right)$ then $\varpi, \varpi^{\prime \prime} \in B$, and $\varpi$ is connected to $\varpi^{\prime \prime}$ by a path contained in $B \subseteq \Pi_{n, \bar{n}}^{-1}\left(\mathcal{B}_{1} \cup \mathcal{B}_{2}\right)$. Since $\varpi \notin \Pi_{n, \bar{n}}^{-1}\left(\mathcal{B}_{2}\right)$ and $\varpi^{\prime \prime} \in \Pi_{n, \bar{n}}^{-1}\left(\mathcal{B}_{2}\right)$, then $\varpi$ is connected in $\Pi_{n, \bar{n}}^{-1}\left(\mathcal{B}_{1}\right)$ to the first point in the path belonging to $\Pi_{n, \bar{n}}^{-1}\left(\mathcal{B}_{2}\right)$.

As previously stated, every $n$-fractal triple with $n>1$ satisfies i) in the definition of C.I.F.T . Thus, if for some $\bar{n}>1$ ii) in the definition of C.I.F.T is satisfied, then we obtain, by Theorem 4.2 (or Corollary 4.3 ) a $G$-eigenform on the corresponding $\bar{n}$-fractal triple. Note that if

$$
\mathcal{A}_{3,1} \backslash\{j\} \text { is connected } \forall j=1, \ldots, N,
$$

then $\mathcal{F}_{2}$ satisfies ii) in Definition of C.I.F.T. To see this, we proceed like in the proof of Lemma 5.7. Given $\left(i_{1}, i_{2}\right),\left(i_{1}^{\prime}, i_{2}^{\prime}\right) \in \mathcal{A}_{2,2}$, that is $\left(i_{1}, i_{2}\right)$ and $\left(i_{1}^{\prime}, i_{2}^{\prime}\right)$ not of the form $(j, j)$ with $j=1, \ldots, N$, there exists a path $\left(\varpi_{1}, \ldots, \varpi_{m}\right)$ in $\mathcal{A}_{3,2}$ connecting them, and if such a path passes through a point $(\bar{j}, \bar{j}) \in \mathcal{A}_{1,2}$, and $\varpi_{s_{1}}=\left(\bar{j}, i_{s_{1}}\right)$ and $\varpi_{s_{2}}=\left(\bar{j}, i_{s_{2}}\right)$ are respectively the first and the last point in the path in $\Pi_{2,1}^{-1}(\bar{j})$, by Lemma 5.5 we have $i_{s_{1}} \neq \bar{j} \neq i_{s_{2}}$, so that by the hypothesis $(5.3)$, we can replace $\left(\varpi_{s_{1}}, \ldots, \varpi_{s_{2}}\right)$ by a path in $\Pi_{2,1}^{-1}(\bar{j})$ not passing through $(\bar{j}, \bar{j})$. By iterating this process, we find a path connecting $\left(i_{1}, i_{2}\right)$ and $\left(i_{1}^{\prime}, i_{2}^{\prime}\right)$ all contained in $\mathcal{A}_{2,2}$. However, if we do not assume (5.3), then in general, ii) in definition of C.I.F.T need not necessarily hold for some $n$, for example in the tree-like Gasket, that is the modified Gasket, with two of the three 1-cells separated, for every $n>1$ the set $\mathcal{A}_{2, n}$ is disconnected, namely has two components. Thus, in order to obtain the main Theorem, we cannot apply Theorem 4.2 (at least directly). We instead will split $\mathcal{A}_{2, n}$ into components and work separately on every component.

For $n>1$, we consider $\mathcal{A}_{2, n}(\mathcal{F})$ as the union of its components $C_{1, n}, \ldots, C_{h_{n}, n}, h_{n} \geq 1$. For every subset $C$ of $\mathcal{A}_{2, n}(\mathcal{F})$ put

$$
(E x)_{n}(C)=\bigcup\left\{C_{i, n}: C_{i, n} \cap C \neq \varnothing\right\} .
$$

In particular, if $C$ is a nonempty subset of a component $C_{l, n}$, and this occurs if $C$ is a nonempty connected subset of $\mathcal{A}_{2, n}$, then $(E x)_{n}(C)=C_{l, n}$. Also, for every $C \subseteq \mathcal{A}_{2, n}$, put

$$
\theta_{n}(C)=\left\{j_{(n)} \in \mathcal{A}_{1, n}: V\left((E x)_{n}(C)\right) \cap V_{j_{(n)}} \neq \varnothing\right\}
$$

Remark 5.9. We easily see that $\theta_{n}(C)$ is the set of elements of $\mathcal{A}_{1, n}(\mathcal{F})$ connected to some point of $C$ in $\mathcal{A}_{2, n}(\mathcal{F})$. 
Lemma 5.10. If $n>1$

i) The set $\theta_{n}(C)$ is nonempty for every nonempty subset $C$ of $\mathcal{A}_{2, n}$

ii) We have $\mathcal{A}_{1, n}(\mathcal{F})=\bigcup\left\{\theta_{n}\left(C_{l, n}\right): \#\left(\theta_{n}\left(C_{l, n}\right)\right) \geq 2\right\}$.

iii) Given $\varnothing \subsetneq \mathcal{A} \subsetneq \mathcal{A}_{1, n}$, there exist $\varpi \in \mathcal{A}, \varpi^{\prime} \in \mathcal{A}_{1, n} \backslash \mathcal{A}$ belonging to the same $\theta_{n}\left(C_{l, n}\right)$.

Proof. Given $\varpi \in C$, take a path connecting $\varpi$ to an element of $\mathcal{A}_{1, n}$. Then, the first element of $\mathcal{A}_{1, n}$ in such a path is in $\theta_{n}(C)$ and i) is proved. For every $\varpi \in \mathcal{A}_{1, n}$, there exists a simple path in $\mathcal{G}_{n}$ connecting $\varpi$ to another element of $\mathcal{A}_{1, n}$. Let $\varpi^{\prime}$ be the first element of $\mathcal{A}_{1, n}$ in such a path different from $\varpi$. The second element $\varpi^{\prime \prime}$ in such a path satisfies $\varpi^{\prime \prime} \sim \varpi$, therefore belongs to $\mathcal{A}_{2, n}$ by Lemma 5.5 , thus to $C_{l, n}$ for some $l$. Thus, $\varpi, \varpi^{\prime} \in \theta_{n}\left(C_{l, n}\right)$, and this proves ii). We can prove similarly iii). Consider a path in $\mathcal{G}_{n}$ connecting an element of $\mathcal{A}$ and an element of $\mathcal{A}_{1, n} \backslash \mathcal{A}$, we take $\varpi$ and $\varpi^{\prime}$ to be two consecutive elements of $\mathcal{A}_{1, n}$ in the path, one in $\mathcal{A}$ and the other in $\mathcal{A}_{1, n} \backslash \mathcal{A}$, and proceed as in ii).

We previously introduced the components $C_{l, n}$ of $\mathcal{A}_{2, n}$, Now, we introduced two types of sets related to them, that is we start from the basic level 2 (we avoid 1 since some results, as Lemma 5.10, require $n>1$ ). Then, for $n \geq 2$, we define the sets $\bar{C}_{l, n}$ and $\widetilde{C}_{l, n}$, that, roughly speaking, represent respectively, the elements of $\mathcal{A}_{2, n}$ and of $\mathcal{A}_{2,2}$ connected to $C_{l, n}$ via $\mathcal{A}_{2, n}$. So, for $n>1$, let

$$
\begin{gathered}
\bar{C}_{l, n}:=(E x)_{n}\left(\Pi_{n, 2}^{-1}\left(C_{l, 2}\right)\right)\left(\subseteq \mathcal{A}_{2, n}\right) \\
\widetilde{C}_{l, n}:=\Pi_{n, 2}\left(\bar{C}_{l, n}\right) \cap \mathcal{A}_{2,2}, \quad B_{l, n}:=\theta_{2}\left(\widetilde{C}_{l, n}\right) \subseteq \mathcal{A}_{1,2}, \quad l=1, \ldots, h_{2} .
\end{gathered}
$$

In general, for fixed $n$, the sets $B_{l, n}$ are not necessarily mutually disjoint, and even some of them could coincide.

Remark 5.11. Note that since $\Pi_{n, 2}^{-1}\left(C_{l, 2}\right)$ is connected by Remark 5.7 , then $\bar{C}_{l, n}$ is a component of $\mathcal{A}_{2, n}$, and $\bar{C}_{l, n} \supseteq \Pi_{n, 2}^{-1}\left(C_{l, 2}\right)$.

Remark 5.12. Given $\varpi \in \mathcal{A}_{2,2}$, then $\varpi \in \widetilde{C}_{l, n}$ if and only if there exist ${ }_{n} \varpi \in \Pi_{n, 2}^{-1}(\varpi)$, ${ }_{n} \varpi^{\prime} \in \Pi_{n, 2}^{-1}\left(C_{l, 2}\right)$ such that ${ }_{n} \varpi$ and ${ }_{n} \varpi^{\prime}$ are connected in $\mathcal{A}_{2, n}$. In such a case, taking

$$
{ }_{n+1} \varpi \in \Pi_{n+1, n}^{-1}\left({ }_{n} \varpi\right) \subseteq \Pi_{n+1,2}^{-1}(\varpi), \quad{ }_{n+1} \varpi^{\prime} \in \Pi_{n+1, n}^{-1}\left({ }_{n} \varpi^{\prime}\right) \subseteq \Pi_{n+1,2}^{-1}\left(C_{l, 2}\right)
$$

then ${ }_{n+1} \varpi$ and ${ }_{n+1} \varpi^{\prime}$ are connected in $\mathcal{A}_{2, n+1}$. As a consequence, $\widetilde{C}_{l, n} \subseteq \widetilde{C}_{l, n+1}$. In particular, $\widetilde{C}_{l, n} \supseteq \widetilde{C}_{l, 2}=C_{l, 2} \neq \varnothing$.

Lemma 5.13. Suppose $n>\bar{n} \geq 2$. Then

i) We have $\Pi_{n, \bar{n}}^{-1}\left(\bar{C}_{l, \bar{n}}\right) \subseteq \bar{C}_{l, n}$,

ii) We have $V\left(\bar{C}_{l, \bar{n}}\right) \cap V\left(\left\{j_{(\bar{n})}\right\}\right) \subseteq V\left(\bar{C}_{l, n} \cap \Pi_{n, \bar{n}}^{-1}\left(j_{(\bar{n})}\right)\right)$ for every $j=1, \ldots, N$. 
Proof. The set $\Pi_{n, \bar{n}}^{-1}\left(\bar{C}_{l, \bar{n}}\right)$ is connected and contains $\Pi_{n, 2}^{-1}\left(C_{l, 2}\right)$, hence is contained in $\bar{C}_{l, n}$ and i) is proved. We now prove ii). Let $Q \in V\left(\bar{C}_{l, \bar{n}}\right) \cap V\left(\left\{j_{(\bar{n})}\right\}\right)$, so that $Q=\psi_{\varpi}\left(P_{h}\right)=$ $\psi_{j_{(\bar{n})}}\left(P_{h^{\prime}}\right)$ for some $\varpi \in \bar{C}_{l, \bar{n}}, h, h^{\prime}=1, \ldots, N$. As $Q \notin V^{(0)}$, we have $h^{\prime} \neq j$. Moreover,

$$
Q=\psi_{\left(\varpi, h_{(n-\bar{n})}\right)}\left(P_{h}\right)=\psi_{\left(j_{(\bar{n})}, h_{(n-\bar{n})}^{\prime}\right)}\left(P_{h^{\prime}}\right),
$$

and $\left(\varpi, h_{(n-\bar{n})}\right) \in \Pi_{n, \bar{n}}^{-1}\left(\bar{C}_{l, \bar{n}}\right) \subseteq \bar{C}_{l, n}$ by i). Since $\left(\varpi, h_{(n-\bar{n})}\right) \sim\left(j_{(\bar{n})}, h_{(n-\bar{n})}^{\prime}\right)$ and $\bar{C}_{l, n}$ is a component in $\mathcal{A}_{2, n}$, and $\left(j_{(\bar{n})}, h_{(n-\bar{n})}^{\prime}\right) \in \mathcal{A}_{2, n}$ as $n-\bar{n}>0$, we have $\left(j_{(\bar{n})}, h_{(n-\bar{n})}^{\prime}\right) \in \bar{C}_{l, n}$, so that $\left(j_{(\bar{n})}, h_{(n-\bar{n})}^{\prime}\right) \in \bar{C}_{l, n} \cap \Pi_{n, \bar{n}}^{-1}\left(j_{(\bar{n})}\right)$, and by (5.4) we conclude.

Lemma 5.14. When $n>1$

i) The sets $B_{l, n}$ are nonempty,

ii) $\left.\mathcal{A}_{1,2}(\mathcal{F})=\bigcup\left\{B_{l, n}: \#\left(B_{l, n}\right)\right) \geq 2\right\}$.

iii) Given $\varnothing \subsetneq \mathcal{A} \subsetneq \mathcal{A}_{1,2}$, there exist $\varpi \in \mathcal{A}, \varpi^{\prime} \in \mathcal{A}_{1,2} \backslash \mathcal{A}$ belonging to the same $B_{l, n}$.

Proof. As $B_{l, n} \supseteq \theta_{2}\left(C_{l, 2}\right)$, i), ii) and iii) follow from the corresponding statement in Lemma 5.10 .

\section{Special subsets of $\mathcal{A}_{3, n}$.}

We now want to mimic the argument in Section 4, working separately on the components. To this aim, we will find a partition of $\mathcal{A}_{3, n}$ of a special type which we will call decomposition of $\mathcal{A}_{3, n}$ into $n$-blocks. By this we will mean a family of subsets $\bar{C}_{l, n}, l=1, \ldots, h^{\prime}$ of $\mathcal{A}_{3, n}$ with the following properties

$$
\begin{gathered}
\#\left(\bar{C}_{l, n} \cap \mathcal{A}_{1, n}\right) \geq 2 \\
\bigcup_{l=1}^{h^{\prime}} \bar{C}_{l, n} \supseteq \mathcal{A}_{1, n} \\
\bar{C}_{\bar{l}+1, n} \cap\left(\bigcup_{l=1}^{\bar{l}} \bar{C}_{l, n}\right)=\left\{\left(\tilde{j}_{l}\right)_{n}\right\} \subseteq \mathcal{A}_{1, n} \\
\bar{C}_{l, n} \cap \mathcal{A}_{2, n} \text { is connected. }
\end{gathered}
$$

In this context, I will call $n$-blocks the sets $\bar{C}_{l, n}$. Note that I have not required that the union of all $\bar{C}_{l, n}$ amounts to all of $\mathcal{A}_{3, n}$, as it will turn out to be impossible to have such a property, keeping at the same time nice properties on the decomposition. Thus, we have to add a possibly nonempty (residual) set in order to obtain a partition of $\mathcal{A}_{3, n}$. The present Section will be devoted to the construction and the main properties of such a decomposition. Once we have a decomposition into $n$-blocks as above, the idea consists in mimicking on every $n$-block the construction of Section 4 . In such a case, of course, we have to adapt the weights in the joint points $\left(\tilde{j}_{l}\right)_{n}$ to two (or more) different $n$-blocks. 
However, this leads to a difficulty. In fact, the effective resistance between $P_{\tilde{j}_{l}}$ and the interior cells of the $n$-block in general depends on the $n$-block, thus unlike the case of Section 4 , we cannot put a weight on $V_{\left(\tilde{j}_{l}\right)_{n}}$ in such a way that such a resistance is, say, $\frac{a_{l}}{2}$ where $a_{l}$ is a suitable positive constant depending on the $n$-block $\bar{C}_{l, n}$. We will modify thus the construction, selecting on every $n$-block some "central" cells, that I will call strictly interior cells among the interior cells and constructing on the $n$-block separate "bridges" between every vertex cell and the strictly interior cells. Next, we will split the bridges into two parts, one exterior, that is closer to the vertex cell, and the interior part, that is closer to the strictly interior cells. Such a construction requires a sufficiently large level $n$. Finally, we will put relatively large weights on the vertex cells in such a way that the effective resistance $R_{1}$ between $P_{j}$ and the bridge is less than $\frac{a_{l}}{2}$ (for every $n$-block the vertex cell belongs to), then weights on the interior part of the bridge such that the effective resistance $R_{2}$ between the exterior part of the bridge and the strictly interior cells satisfies

$$
R_{1}+R_{2}=\frac{a_{l}}{2}
$$

thus, putting large weights on the exterior part of the bridge, using Lemma 3.7, the effective resistance between $P_{\tilde{j}_{l}}$ and the strictly interior cells of the $n$-block approximates $\frac{a_{l}}{2}$. Thus, putting large weights on the strictly interior cells of the $n$-block, we can proceed like in Section 4. However, there is a further difficulty, that is, we want that the eigenform is regular, and this occurs if the weights on all the interior cells of $\mathcal{F}_{n}$, including that in the bridges, are not less than the weights on the vertex cells (cf. Remark 4.4). On the other hand, if $R_{1}$ is small, then formula $R_{1}+R_{2}=\frac{a_{l}}{2}$ cannot hold when the weights on the interior part of the bridge is not sufficiently small. To overcame such a difficulty, we will fill the cells, choosing a sufficiently large level $n$, as the effective resistance goes to infinity when the level $n$ goes to infinity (cf. Lemma 7.8 and Corollary 7.9). For a similar reason, when the level is sufficiently large the residual set, in the initial construction, is negligible. Although the construction in the sequel could differ in some details from the ideas above discussed, this motivates the next definitions. First of all, we will suitably split the set $\mathcal{A}_{1, n}$. Note that formulas $(3.1),(3.2),(3.3)$ below are related respectively to $\left(P_{1}\right),\left(P_{2}\right)$, $\left(P_{3}\right)$.

By Remark 5.12 there exists $n_{1}>2$ such that

$$
\widetilde{C}_{l, n_{1}}=\widetilde{C}_{l, n} \quad \text { hence } B_{l, n}=B_{l, n_{1}} \quad \forall n \geq n_{1} .
$$

We put $\widetilde{C}_{l}=\widetilde{C}_{l, n_{1}}, B_{l}=B_{l, n_{1}}$. In view of Lemma 5.14 iii) with $\mathcal{A}=\bigcup_{s<l} B_{s}$, we can also enumerate the sets $B_{l}, l \leq h^{\prime} \leq h_{2}$, in such a way that

$$
\begin{gathered}
\#\left(B_{1}\right) \geq 2, \\
B_{l+1} \nsubseteq \bigcup_{s=1}^{l} B_{s}, \quad x_{l} \in\left(\bigcup_{s=1}^{l} B_{s}\right) \cap B_{l+1}, \quad \bigcup_{l=1}^{h^{\prime}} B_{l}=\mathcal{A}_{1,2} \quad \forall l=1, \ldots, h^{\prime}-1, \\
x_{l}=\left(\tilde{j}_{l}, \tilde{j}_{l}\right), \quad \tilde{j}_{l}=1, \ldots, N .
\end{gathered}
$$


Remark 6.1. Note that, using the previous enumeration of $B_{l}$, for every $n \geq n_{1}$ the sets $\bar{C}_{l, n}, l=1, \ldots, h^{\prime}$, are distinct, hence mutually disjoint, and, as they are components in $\mathcal{A}_{2, n}$, they are in fact mutually separated.

We define $B_{l}^{\prime}, \widetilde{B}_{l}^{\prime}$ by:

$$
\begin{gathered}
B_{1}^{\prime}=B_{1}, \quad B_{l+1}^{\prime}=\left(B_{l+1} \backslash\left(\bigcup_{s=1}^{l} B_{s}\right)\right) \cup\left\{x_{l}\right\}, \quad 1 \leq l \leq h^{\prime}-1 . \\
\widetilde{B}_{l}^{\prime}:=\Pi_{2,1}\left(B_{l}^{\prime}\right)=\left\{j=1, \ldots, N:(j, j) \in B_{l}^{\prime}\right\},
\end{gathered}
$$

in other words, $j \in \widetilde{B}_{l}^{\prime} \Longleftrightarrow(j, j) \in B_{l}^{\prime}$. We have $B_{l}^{\prime} \subseteq B_{l}$, and also,

$$
\begin{gathered}
\#\left(\widetilde{B}_{l}^{\prime}\right)=\#\left(B_{l}^{\prime}\right) \geq 2, \\
\bigcup_{s=1}^{l} B_{s}^{\prime}=\bigcup_{s=1}^{l} B_{s} \quad \forall l=1, \ldots, h^{\prime}, \\
\left(\bigcup_{s=1}^{l} B_{s}^{\prime}\right) \cap B_{l+1}^{\prime}=\left\{x_{l}\right\} \quad \forall l=2, \ldots, h^{\prime}, \\
\left(\bigcup_{s=1}^{l} \widetilde{B}_{s}^{\prime}\right) \cap \widetilde{B}_{l+1}^{\prime}=\left\{\tilde{j}_{l}\right\} \quad \forall l=2, \ldots, h^{\prime},
\end{gathered}
$$

so that $\#\left(B_{l_{1}}^{\prime} \cap B_{l_{2}}^{\prime}\right) \leq 1$ when $l_{1} \neq l_{2}$. As a particular case of $(6.2), \bigcup_{s=1}^{h^{\prime}} B_{s}^{\prime}=\mathcal{A}_{1,2}$, and consequently, $\bigcup_{s=1}^{h^{\prime}} \widetilde{B}_{s}^{\prime}=\{1, \ldots, N\}$. Let

$$
\bar{J}=: \bigcup_{l=1}^{h^{\prime}} J\left(\widetilde{B}_{l}^{\prime}\right) \subseteq J
$$

We will now construct the $n$-blocks for $n=n_{2}$ (the sets $\bar{C}_{l}^{(4)}$ ) and also for $n>n_{2}$ (the sets $\bar{G}_{l, n}^{(4)}$ ), where $n_{2}$ is a suitable integer with $n_{2}>n_{1}+1$. The sets $\bar{C}_{j}^{(1)}$ (or more precisely $\left.V\left(\bar{C}_{j}^{(1)}\right)\right)$ represent the vertex cells, the sets $\bar{C}_{l}^{(3)}$ the strictly interior cells and the sets $\bar{C}_{j, l}^{(2)}$ the bridges. So, fix $n_{2}>n_{1}+1$ and for every $j \in \widetilde{B}_{l}^{\prime}, l=1, \ldots, h^{\prime}$, and $n \geq n_{2}$, define the following subsets of $\mathcal{A}_{3, n_{2}}$,

$$
\begin{aligned}
& \bar{C}_{j}^{(1)}=\left\{j_{\left(n_{2}\right)}\right\}, \\
& \bar{C}_{j, l}^{(2)}=\bar{C}_{l, n_{2}} \cap \Pi_{n_{2}, n_{1}}^{-1}\left(j_{\left(n_{1}\right)}\right), \\
& \bar{C}_{l}^{(3)}=\Pi_{n_{2}, n_{1}}^{-1}\left(\bar{C}_{l, n_{1}}\right),
\end{aligned}
$$

$$
\begin{aligned}
& \bar{G}_{j, n}^{(1)}=\Pi_{n, n_{2}}^{-1}\left(\bar{C}_{j}^{(1)}\right) \\
& \bar{G}_{j, l, n}^{(2)}=\Pi_{n, n_{2}}^{-1}\left(\bar{C}_{j, l}^{(2)}\right) \\
& \bar{G}_{l, n}^{(3)}=\Pi_{n, n_{2}}^{-1}\left(\bar{C}_{l}^{(3)}\right)=\Pi_{n, n_{1}}^{-1}\left(\bar{C}_{l, n_{1}}\right)
\end{aligned}
$$


In the sequel, I will define other sets of the form $\bar{C}_{b}^{(a)}$ where $a$ and $b$ are symbols or strings of symbols. I will use the general convention that in such a case $\bar{G}_{b, n}^{(a)}=\Pi_{n, n_{2}}^{-1}\left(\bar{C}_{b}^{(a)}\right)$. In order to apply Lemma 3.7 as hinted above, the exterior part of the bridge should be connected (note that we also need the connectedness of $\bar{C}_{l}^{(3)}$, but this immediately follows from Lemma 5.7 and Remark 5.11). Thus, I will first modify every bridge $\bar{C}_{j, l}^{(2)}$, extracting a connected subset of it, and then I will split such a connected set into two parts, the exterior part $C_{j, l}^{(21)}$ and the interior part $C_{j, l}^{(22)}$ according to the hinted idea. We need the following Lemma.

Lemma 6.2. There exists a simple path $\left(\bar{\varpi}_{0}, \ldots, \bar{\varpi}_{\bar{m}}\right)$ connecting $j_{\left(n_{2}\right)}$ to some element of $\bar{C}_{l}^{(3)}$ in $\bar{C}_{j, l}^{(2)}$.

Proof. For every $l=1, \ldots, h^{\prime}$, if $j \in \widetilde{B}_{l}^{\prime}$, then $j_{(2)}$ is connected to an element of $\widetilde{C}_{l}$ in $\left\{j_{(2)}\right\} \cup$ $\widetilde{C}_{l}$. By Lemma $5.8, j_{\left(n_{1}\right)} \in \Pi_{n_{1}, 2}^{-1}\left(j_{(2)}\right)$ is connected in $\Pi_{n_{1}, 2}^{-1}\left(j_{(2)}\right)$ to some ${ }_{n_{1}} \varpi \in \Pi_{n_{1}, 2}^{-1}\left(\widetilde{C}_{l}\right)$. Moreover, by the definition of $\widetilde{C}_{l}$, there exists $n_{1} \varpi^{\prime} \in \bar{C}_{l, n_{1}}$ such that $\Pi_{n_{1}, 2}\left(n_{1} \varpi^{\prime}\right)=$ $\Pi_{n_{1}, 2}\left({ }_{n_{1}} \varpi\right):=\varpi \in \widetilde{C}_{l} \subseteq \mathcal{A}_{2,2}$, hence, as $\Pi_{n_{1}, 2}^{-1}(\varpi)$ is connected and contained in $\mathcal{A}_{2, n_{1}}$, $j_{\left(n_{1}\right)}$ is connected to ${ }_{n_{1}} \varpi^{\prime}$ by a simple path in $\Pi_{n_{1}, 2}^{-1}\left(j_{(2)}\right) \cup \mathcal{A}_{2, n_{1}}$. Also, as every element of $\Pi_{n_{1}, 2}^{-1}\left(j_{(2)}\right)$ different from $j_{\left(n_{1}\right)}$ lies in $\mathcal{A}_{2, n_{1}}$, the elements of the path but the first are thus in $\mathcal{A}_{2, n_{1}}$, hence, by the definition of $\bar{C}_{l, n_{1}}$, they are in fact in $\bar{C}_{l, n_{1}}$, so that $j_{\left(n_{1}\right)}$ is connected to ${ }_{n_{1}} \varpi^{\prime}$ in $\left\{j_{\left(n_{1}\right)}\right\} \cup \bar{C}_{l, n_{1}}$. By Lemma 5.8 again, $j_{\left(n_{2}\right)} \in \Pi_{n_{2}, n_{1}}^{-1}\left(\left\{j_{\left(n_{1}\right)}\right\}\right)$ is connected to some point of $\Pi_{n_{2}, n_{1}}^{-1}\left(\bar{C}_{l, n_{1}}\right)=\bar{C}_{l}^{(3)}$ by a simple path in $\Pi_{n_{2}, n_{1}}^{-1}\left(\left\{j_{\left(n_{1}\right)}\right\}\right)$, and as the elements of the path but the first are in $\mathcal{A}_{2, n_{2}}$, and the last is in $\Pi_{n_{2}, n_{1}}^{-1}\left(\bar{C}_{l, n_{1}}\right) \subseteq \bar{C}_{l, n_{2}}$, then the path is in $\bar{C}_{l, n_{2}} \cap \Pi_{n_{2}, n_{1}}^{-1}\left(\left\{j_{\left(n_{1}\right)}\right\}\right)=\bar{C}_{j, l}^{(2)}$.

Now, we consider $\bar{\varpi}$ as in the Lemma and we can and do assume that this path is of minimum length, so that

$$
\text { if } s^{\prime}>s+1 \text { then } V_{\bar{\varpi}_{s^{\prime}}} \cap V_{\bar{\varpi}_{s}}=\varnothing \text {. }
$$

We have $\bar{\varpi}_{0}=j_{\left(n_{2}\right)}$ and $\bar{\varpi}_{\bar{m}} \notin \Pi_{n_{2}, n_{1}}^{-1}\left(j_{\left(n_{1}\right)}\right)$ thus, by Corollary 5.5 , we have $\Pi_{n_{2}, n_{2}-1}\left(\bar{\varpi}_{1}\right)$ $=j_{\left(n_{2}-1\right)}$, and recalling that $n_{2}-1 \geq n_{1}+1, \Pi_{n_{2}, n_{1}+1}\left(\bar{\varpi}_{1}\right)=j_{\left(n_{1}+1\right)}$, thus

$$
\bar{\varpi}_{1} \in \Pi_{n_{2}, n_{1}+1}^{-1}\left(j_{\left(n_{1}+1\right)}\right), \quad \bar{\varpi}_{\bar{m}-1} \notin \Pi_{n_{2}, n_{1}+1}^{-1}\left(j_{\left(n_{1}+1\right)}\right)
$$

Now, let $\tilde{s}$ be the maximum index $s$ so that $\bar{\varpi}_{s} \in \Pi_{n_{2}, n_{1}+1}^{-1}\left(j_{\left(n_{1}+1\right)}\right)$, and put

$$
\bar{C}_{j, l}^{(21)}=\left\{\bar{\varpi}_{s}: 1 \leq s \leq \tilde{s}\right\} \subseteq \bar{C}_{j, l}^{(2)}, \quad \bar{C}_{j, l}^{(22)}=\left\{\bar{\varpi}_{s}: \tilde{s}<s<\bar{m}\right\} \subseteq \bar{C}_{j, l}^{(2)}
$$

and define $\bar{G}_{j, l, n}^{(21)}, \bar{G}_{j, l, n}^{(22)}$ according to the previous convention. Moreover, note that $1 \leq$ $\tilde{s}<\bar{m}-1$, and $\bar{\varpi}_{\tilde{s}} \in \bar{C}_{j, l}^{(21)}, \bar{\varpi}_{\tilde{s}+1} \in \bar{C}_{j, l}^{(22)}$. We have

$$
V\left(\bar{C}_{j, l}^{(21)}\right) \cap V\left(\bar{C}_{j, l}^{(22)}\right)=V_{\bar{\varpi}_{\tilde{s}}} \cap V_{\bar{\varpi}_{\tilde{s}+1}} .
$$


In fact, the inclusion $\supseteq$ is trivial. To prove $\subseteq$, note that if $Q \in V\left(\bar{C}_{j, l}^{(21)}\right) \cap V\left(\bar{C}_{j, l}^{(22)}\right)$, then there exist $s \leq \tilde{s}, s^{\prime}>\tilde{s}$ such that $Q \in V_{\bar{\varpi}_{s}} \cap V_{\bar{\varpi}_{s^{\prime}}}$. On the other hand, by (6.4), we have $s=\tilde{s}$ and $s^{\prime}=\tilde{s}+1$. For $j \in \widetilde{B}_{l}^{\prime}, l=1, \ldots, h^{\prime}$, put

$$
\begin{gathered}
\bar{C}_{l}^{(4)}=\left(\bigcup_{j \in \widetilde{B}_{l}^{\prime}} \bar{C}_{j}^{(1)}\right) \cup\left(\bigcup_{j \in \widetilde{B}_{l}^{\prime}}\left(\bar{C}_{j, l}^{(21)} \cup \bar{C}_{j, l}^{(22)}\right)\right) \cup \bar{C}_{l}^{(3)} \\
\bar{C}^{(5)}=\bigcup_{l=1}^{h^{\prime}} \bar{C}_{l}^{(4)} \\
\bar{C}^{(6)}=\mathcal{A}_{3, n_{2}} \backslash \bar{C}^{(5)}
\end{gathered}
$$

For every $j \in \widetilde{B}_{l}^{\prime}$ let $\bar{C}_{j, l}^{(7)}$ be the set of $\varpi \in \bar{C}^{(5)} \backslash \bar{C}_{l}^{(4)}$ connected to $j_{\left(n_{2}\right)}$ in $\bar{C}^{(5)} \backslash \bar{C}_{l}^{(4)}$. We of course define $\bar{G}_{l, n}^{(4)}, \bar{G}_{n}^{(5)}, \bar{G}_{n}^{(6)}$, and $\bar{G}_{j, l, n}^{(7)}=\Pi_{n, n_{2}}^{-1}\left(\bar{C}_{j, l}^{(7)}\right)$ as usual. The sets $\bar{C}_{l}^{(4)}$ are the $n_{2}$-blocks, and $\bar{C}^{(6)}$ is the residual set, and $\bar{G}_{l, n}^{(4)}$ and $\bar{G}_{n}^{(6)}$ are the analogs at level $n$. Using also Lemma 6.3 ii) below, we easily see that properties $\left(P_{1}\right),\left(P_{2}\right),\left(P_{3}\right),\left(P_{4}\right)$ hold.

We remark that the sets of the forms $\bar{C}_{j, l}^{(21)}, \bar{C}_{j, l}^{(22)}, \bar{C}_{l}^{(3)}$ are contained in $\mathcal{A}_{2, n_{2}}$ and consequently $\bar{G}_{j, l, n}^{(21)}, \bar{G}_{j, l, n}^{(22)}, \bar{G}_{l, n}^{(3)}$ are contained in $\mathcal{A}_{2, n}$. On the contrary, $\bar{C}_{j}^{(1)}$ is contained in $\mathcal{A}_{1, n_{2}}$.

In the following lemmas, we will now see the intersection properties of the sets defined above. In particular, the sets with one of apex (1), (21), (22) and (3) are mutually disjoint.

\section{Lemma 6.3.}

i) The sets $\bar{C}_{j}^{(1)}, \bar{C}_{j, l}^{(21)}, \bar{C}_{j, l}^{(22)}, \bar{C}_{l}^{(3)}$ are mutually disjoint. Consequently, for fixed $n \geq n_{2}$, so are the sets $\bar{G}_{j, n}^{(1)}, \bar{G}_{j, l, n}^{(21)}, \bar{G}_{j, l, n}^{(22)} \bar{G}_{l, n}^{(3)}$.

ii) Suppose $l \neq l^{\prime}$. Then, if $Q \in \bar{C}_{l}^{(4)} \cap \bar{C}_{l^{\prime}}^{(4)}$, we have $Q=j_{\left(n_{2}\right)}$ for some $j \in \widetilde{B}_{l}^{\prime} \cap \widetilde{B}_{l^{\prime}}^{\prime}$.

Proof. In the present proof, we will say that the sets of the form $\bar{C}_{j}^{(1)}$ are of type 1 , the sets of the form $\bar{C}_{j, l}^{(2)}$ are of type 2 , the sets of the form $\bar{C}_{l}^{(3)}$ are of type 3 . Using such a convention, the sets of type 1 are contained in $\mathcal{A}_{1, n_{2}}$, the sets of type 2 are contained both in $\mathcal{A}_{2, n_{2}}$ and in $\Pi_{n_{2}, n_{1}}^{-1}\left(\mathcal{A}_{1, n_{1}}\right)$, the sets of type 3 are contained both in $\mathcal{A}_{2, n_{2}}$ and in $\Pi_{n_{2}, n_{1}}^{-1}\left(\mathcal{A}_{2, n_{1}}\right)$. Hence, as $\mathcal{A}_{1, n}$ and $\mathcal{A}_{2, n}$ are disjoint for every fixed $n$, sets of different types are disjoint. Sets of the same type are disjoint as for every $n$ the sets $\left\{j_{(n)}\right\}$ are obviously mutually disjoint and by Remark 6.1. Finally, the sets $\bar{C}_{j, l}^{(21)}$ and $\bar{C}_{j, l}^{(22)}$ are disjoint as, by hypothesis the path $\left(\bar{\varpi}_{0}, \ldots, \bar{\varpi}_{\bar{m}}\right)$ is simple. This proves i), and ii) is a simple consequence of i).

Lemma 6.4. Suppose $\varpi, \varpi^{\prime} \in \bar{C}^{(5)}, V_{\varpi} \cap V_{\varpi^{\prime}} \neq \varnothing$. Then

i) There exists $l$ such that $\varpi, \varpi^{\prime} \in \bar{C}_{l}^{(4)}$.

ii) If $\varpi \in \bar{C}_{l}^{(4)}, \varpi^{\prime} \notin \bar{C}_{l}^{(4)}$, then $\varpi=j_{\left(n_{2}\right)}$ for some $j \in \widetilde{B}_{l}^{\prime}$. 
Proof. We have $\varpi \in \bar{C}_{l}^{(4)}, \varpi^{\prime} \in \bar{C}_{l^{\prime}}^{(4)}$ for some $l, l^{\prime}=1, \ldots, h^{\prime}$. By Remark 6.1, if $V_{\varpi} \cap V_{\varpi^{\prime}} \neq$ $\varnothing$, then, either $\varpi, \varpi^{\prime} \in \bar{C}_{l, n_{2}}$ for some $l$, or one of $\varpi, \varpi^{\prime}$, for example $\varpi^{\prime}$, amounts to $j_{\left(n_{2}\right)}$ for some $j=1, \ldots, N$. In the latter case, by Lemma 5.5 i) $\Pi_{n_{2}, n_{1}}(\varpi)=j_{\left(n_{1}\right)}$, thus the unique possibility is that $\varpi \in \bar{C}_{j, l}^{(2)}$ for some $l$ with $j \in \widetilde{B}_{l}^{\prime}$ by the definition of the $\bar{C}^{(5)}$. It follows that $\varpi, \varpi^{\prime} \in \bar{C}_{l}^{(4)}$. As, by Lemma $6.2, \varpi \notin \bar{C}_{l^{\prime}}^{(4)}$ if $l \neq l^{\prime}$, then i) and ii) easily follow.

\section{Lemma 6.5.}

i) The set $\bar{C}_{j, l}^{(8)}:=\bar{C}_{j}^{(1)} \cup\left(\left(\bar{C}_{j, l}^{(21)} \cup \bar{C}_{j, l}^{(22)}\right)\right) \cup \bar{C}_{l}^{(3)}$ is connected when $j \in \widetilde{B}_{l}^{\prime}$.

ii) The set $\bar{C}_{l}^{(4)}$ is connected for every $l=1, \ldots, h^{\prime}$.

iii) The set $\bar{C}^{(5)}$ is connected.

Proof. Recall that $\bar{C}_{j, l}^{(21)} \cup \bar{C}_{j, l}^{(22)}=\left\{\bar{\varpi}_{s}: 1 \leq s<\bar{m}\right\}$. Thus,

$$
\bar{C}_{j, l}^{(8)}=\left\{\bar{\varpi}_{0}, \ldots, \bar{\varpi}_{\bar{m}}\right\} \cup \bar{C}_{l}^{(3)}, \quad \bar{\varpi}_{\bar{m}} \in\left\{\bar{\varpi}_{0}, \ldots, \bar{\varpi}_{\bar{m}}\right\} \cap \bar{C}_{l}^{(3)}
$$

and, as $\bar{C}_{l}^{(3)}$ is connected by Lemma 5.7, then i) is proved, and, as $\bar{C}_{l}^{(3)} \neq \varnothing$, ii) is an immediate consequence of i). As, by definition, $j_{\left(n_{2}\right)} \in \bar{C}_{l}^{(4)}$ for every $j \in \widetilde{B}_{l}^{\prime}$, and by (6.3), $\left(\tilde{j}_{l}\right)_{\left(n_{2}\right)} \in\left(\bigcup_{s=1}^{l} \bar{C}_{s}^{(4)}\right) \cap \bar{C}_{l+1}^{(4)} \neq \varnothing$ for every $l=1, \ldots, h^{\prime}-1$, iii) easily follows from ii).

I previously introduced the sets $\bar{C}_{j, l}^{(7)}$. In some sense, such sets can be seen as "tails" attached to every "boundary" point $j_{\left(n_{2}\right)}$ of $\bar{C}_{l}^{(4}$. We now discuss the structure of such sets. In particular, Lemma 6.6 i) states that $\bar{C}^{(5)}$ can be represented as $\bar{C}_{l}^{(4)}$ with the addition of the separate tails $\bar{C}_{j, l}^{(7)}$.

Lemma 6.6.

i) The set $\bar{C}^{(5)} \backslash \bar{C}_{l}^{(4)}$ is the union of the mutually disjoint sets $\bar{C}_{j, l}^{(7)}, j \in \widetilde{B}_{l}^{\prime}$.

ii) For fixed $l=1, \ldots, h^{\prime}$, the sets $\bar{C}_{j}^{(1)}, \bar{C}_{j, l}^{(7)}, \bar{C}_{j, l}^{(21)}, \bar{C}_{j, l}^{(22)}, \bar{C}_{l}^{(3)}, j \in \widetilde{B}_{l}^{\prime}$ are mutually disjoint and there union amounts to $\bar{C}^{(5)}$. Consequently, the sets $\bar{G}_{j, n}^{(1)}, \bar{G}_{j, l, n}^{(7)}, \bar{G}_{j, l, n}^{(21)}, \bar{G}_{j, l, n}^{(22)}, \bar{G}_{l, n}^{(3)}$, $j \in \widetilde{B}_{l}^{\prime}$ are mutually disjoint and there union amounts to $\bar{G}_{n}^{(5)}$.

Proof. We first prove that

$$
\bar{C}_{j, l}^{(7)} \cap \bar{C}_{j^{\prime}, l}^{(7)}=\varnothing \quad \forall j, j^{\prime} \in \widetilde{B}_{l}^{\prime} \mid j \neq j^{\prime} .
$$

If this is false, then there exists a simple path $y_{1}=j_{\left(n_{2}\right)}, y_{2}, \ldots, y_{s}=j_{\left(n_{2}\right)}^{\prime}$, with $s>2$,

$$
y_{i} \in \bar{C}^{(5)} \backslash \bar{C}_{l}^{(4)} \text { when } 1<i<s .
$$

Let $\bar{l}$ be the maximum $m$ such that $\bar{C}_{m}^{(4)}$ contains at least two different $y_{i}$, namely $y_{i_{1}}$ and $y_{i_{2}}$. Suppose $i_{1}, i_{2}$ are respectively the minimum and the maximum of the set of the indices 
$i$ such that $y_{i} \in \bar{C}_{\bar{l}}^{(4)}$. As $y_{1}, y_{s} \in \bar{C}_{l}^{(4)}$, then $\bar{l} \geq l$. If $\bar{l}=l$, then $i_{1}=1$ and $i_{2}=s$. Thus, by (6.7) and Lemma 6.4 we have

$$
y_{i_{1}}, y_{i_{2}} \in \bar{C}_{\bar{l}}^{(4)} \cap\left(\bigcup_{m<\bar{l}} \bar{C}_{m}^{(4)}\right) .
$$

If, on the contrary, $\bar{l}>l$, then either $i_{1}=1$ and $y_{i_{1}} \in \bar{C}_{\bar{l}}^{(4)} \cap \bar{C}_{l}^{(4)}$, or $i_{1}>1$ and $y_{i_{1}-1} \notin \bar{C}_{\bar{l}}^{(4)}$ by the definition of $i_{1}$, thus, by Lemma 6.4 again we have $y_{i_{1}-1}, y_{i_{1}} \in \bar{C}_{m}^{(4)}$ for some $m \neq \bar{l}$, and by the definition of $\bar{l}$ we have $m<\bar{l}$, thus

$$
y_{i_{1}} \in \bar{C}_{\bar{l}}^{(4)} \cap\left(\bigcup_{m<\bar{l}} \bar{C}_{m}^{(4)}\right)
$$

and a similar argument works for $y_{i_{2}}$ so that (6.8) holds as well. By Lemma 6.3 ii), as $y_{i_{1}} \in \bar{C}_{\bar{l}}^{(4)} \cap \bar{C}_{m_{1}}^{(4)}, y_{i_{2}} \in \bar{C}_{\bar{l}}^{(4)} \cap \bar{C}_{m_{2}}^{(4)}$ with $m_{1}, m_{2}<\bar{l}$, thus $y_{i_{1}}=\left(j_{1}\right)_{n_{2}}, y_{i_{2}}=\left(j_{2}\right)_{n_{2}}$, $j_{1}, j_{2} \in \widetilde{B}_{\bar{l}}^{\prime} \cap\left(\bigcup_{m<\bar{l}} \widetilde{B}_{m}^{\prime}\right)$, and $j_{1} \neq j_{2}$, but this contradicts (6.3). To prove that every point $x$ in $\bar{C}^{(5)} \backslash \bar{C}_{l}^{(4)}$ belongs to some $\bar{C}_{j, l}^{(7)}$, note that, by Lemma 6.4 , there exists a path in $\bar{C}^{(5)} \backslash \bar{C}_{l}^{(4)}$ connecting $x$ to some element of $\bar{C}_{l}^{(4)}$. Let $\varpi_{s-1}, \varpi_{s}$ be the last two points in such a path. As $\varpi_{s-1} \notin \bar{C}_{l}^{(4)}$, while $\varpi_{s} \in \bar{C}_{l}^{(4)}$, and $V_{\varpi_{s-1}} \cap V_{\varpi_{s}} \neq \varnothing$, by Lemma 6.3 , $\varpi_{s}=j_{\left(n_{2}\right)}$ for some $j \in \widetilde{B}_{l}^{\prime}$, thus $x \in \bar{C}_{j, l}^{(7)}$. This proves i). In order to prove ii), note that the first statement follows from Lemma $6.3 \mathrm{i}$ ) and i), and the fact that the $\bar{C}_{l}^{(4)}$ is the union of the sets $\bar{C}_{j}^{(1)}, \bar{C}_{j, l}^{(21)}, \bar{C}_{j, l}^{(22)}, \bar{C}_{l}^{(3)}$ with $j \in \widetilde{B}_{l}^{\prime}$.

Remark 6.7. If $j \in \widetilde{B}_{l}^{\prime}, \varpi \in \bar{G}_{j, l, n}^{(7)}$, and $\left(\varpi_{0}, \ldots \varpi_{m}\right), \varpi_{s} \in \bar{G}_{n}^{(5)}$, is a path connecting $\varpi$ to some element $\varpi^{\prime}$ of $\bar{G}_{l, n}^{(4)}$, then the first element $\varpi_{\bar{s}}$ of the path in $\bar{G}_{l, n}^{(4)}$ in fact belongs to $\bar{G}_{j, n}^{(1)}$. To see this, let ${ }_{n_{2}} \varpi_{i}:=\Pi_{n, n_{2}}\left(\varpi_{i}\right)$. Then, $\left(n_{2} \varpi_{0}, \ldots, n_{2} \varpi_{\bar{s}}\right)$ is a weak path connecting ${ }_{n_{2}} \varpi_{0} \in \bar{C}_{j, l}^{(7)}$ to ${ }_{n_{2}} \varpi_{\bar{s}} \in \bar{C}_{l}^{(4)}$ in $\bar{C}^{(5)} \backslash \bar{C}_{l}^{(4)}$. As $\bar{s} \geq 1$ and ${ }_{n_{2}} \varpi_{\bar{s}-1} \in \bar{C}^{(5)} \backslash \bar{C}_{l}^{(4)}$, $V_{n_{2} \varpi_{\bar{s}}-1} \cap V_{n_{2} \varpi_{\bar{s}}} \neq \varnothing$, by Lemma 6.4 , we have ${ }_{n_{2}} \varpi_{\bar{s}}=j_{\left(n_{2}\right)}^{\prime}$ for some $j^{\prime} \in \widetilde{B}_{l}^{\prime}$, thus ${ }_{n_{2}} \varpi_{0}={ }_{n_{2}} \varpi \in \bar{C}_{j^{\prime}, l}^{(7)}$. By Lemma 6.6, we have $j^{\prime}=j$, thus $\varpi_{\bar{s}} \in \bar{G}_{j, n}^{(1)}$, as claimed. Consequently, if $\varpi \in \bar{G}_{j, l, n}^{(7)}$ and $\varpi^{\prime} \in \bar{G}_{n}^{(5)}, V_{\varpi} \cap V_{\varpi^{\prime}} \neq \varnothing$, then $\varpi^{\prime} \in \bar{G}_{j, n}^{(1)} \cup \bar{G}_{j, l, n}^{(7)}$. Conversely, if $j \in \widetilde{B}_{l}^{\prime}$ and $\left(\varpi_{0}, \ldots \varpi_{m}\right), \varpi_{s} \in \bar{G}_{n}^{(5)}$, is a path connecting $\varpi \notin \bar{G}_{l, n}^{(4)}$ to some element $\varpi^{\prime}$ of $\bar{G}_{l, n}^{(4)}$, and the first element $\varpi_{\bar{s}}$ of the path in $\bar{G}_{l, n}^{(4)}$ in fact belongs to $\bar{G}_{j, n}^{(1)}$, then $\varpi \in \bar{G}_{j, l, n}^{(7)}$. In fact, if ${ }_{n_{2}} \varpi_{s}:=\Pi_{n, n_{2}}\left(\varpi_{s}\right)$, then ${ }_{n_{2}} \varpi_{0}=\Pi_{n, n_{2}}(\varpi) \in \bar{C}^{(5)} \backslash \bar{C}_{l}^{(4)}$ is connected to ${ }_{n_{2}} \varpi_{\bar{s}}=j_{\left(n_{2}\right)}$ in $\bar{C}^{(5)} \backslash \bar{C}_{l}^{(4)}$. Hence ${ }_{n_{2}} \varpi_{0} \in \bar{C}_{j, l}^{(7)}$.

In Lemma 6.3 we saw that the sets $G$ with apex (1), (21), (22) and (3) are mutually disjoint. We will now see when two of these are separated. Namely, roughly speaking, they are always separated, unless thery are trivially nonseparated. 
Lemma 6.8. Let $\bar{G}$ be the family of the sets $\bar{G}_{j, n}^{(1)}, \bar{G}_{j, l, n}^{(21)}, \bar{G}_{j, l, n}^{(22)}, \bar{G}_{l, n}^{(3)}$, with $l=1, \ldots, h^{\prime}$, $j \in \widetilde{B}_{l}^{\prime}$. Then

a) The set $\bar{G}_{j, n}^{(1)}$, is separated from all sets of $\bar{G}$ but itself and $\bar{G}_{j, l, n}^{(21)}$ and the sets with $j \in \widetilde{B}_{l}^{\prime}$

b) The set $\bar{G}_{j, l, n}^{(21)}$ is separated from all sets of $\bar{G}$ but itself, $\bar{G}_{j, n}^{(1)}$ and $\bar{G}_{j, l, n}^{(22)}$

c) The set $\bar{G}_{j, l, n}^{(22)}$ is separated from all sets of $\bar{G}$ but itself, $\bar{G}_{l, n}^{(3)}$ and $\bar{G}_{j, l, n}^{(21)}$

d) The set $\bar{G}_{l, n}^{(3)}$ is separated from all sets of $\bar{G}$ but itself and $\bar{G}_{j, l, n}^{(22)}$

e) The set $\bar{G}_{j, l, n}^{(7)}$ is separated from the sets $\bar{G}_{j, l, n}^{(21)}, \bar{G}_{j, l, n}^{(22)}, \bar{G}_{l, n}^{(3)}$.

Proof. We will prove the following facts

$\left(S_{1}\right)$ The sets $\bar{C}_{j}^{(1)}, \bar{C}_{j, l}^{(21)}, \bar{C}_{j, l}^{(22)}, \quad$ are separated from $\bar{C}_{j^{\prime}}^{(1)}, \bar{C}_{j^{\prime}, l^{\prime}}^{(21)}, \bar{C}_{j^{\prime}, l^{\prime}}^{(22)}$, when $j \neq j^{\prime}$

$\left(S_{2}\right)$ The sets $\bar{C}_{j, l}^{(21)}, \bar{C}_{j, l}^{(22)}, \bar{C}_{l}^{(3)}$ are separated from $\bar{C}_{j^{\prime}, l^{\prime}}^{(21)}, \bar{C}_{j^{\prime}, l^{\prime}}^{(22)}, \bar{C}_{l^{\prime}}^{(3)}$, when $l \neq l^{\prime}$

$\left(S_{3}\right) \bar{C}_{j}^{(1)}$ is separated from $\bar{C}_{j, l}^{(22)}, j \in \widetilde{B}_{l}^{\prime} ; \bar{C}_{l}^{(3)},\left(j\right.$ not necessarily in $\left.\widetilde{B}_{l}^{\prime}\right)$

$\left(S_{4}\right) \bar{C}_{j, l}^{(21)}$ is separated from $\bar{C}_{l}^{(3)}$

and, in view of Remark 5.6, this suffices to conclude. To prove $\left(S_{1}\right)$ it suffices to note that the sets $\bar{C}_{j}^{(1)}, \bar{C}_{j, l}^{(21)}, \bar{C}_{j, l}^{(22)}$ are contained in $\Pi_{n_{2}, n_{1}}^{-1}\left(j_{\left(n_{1}\right)}\right)$, and the sets $\left\{j_{\left(n_{1}\right)}\right\}$ and $\left\{j_{\left(n_{1}\right)}^{\prime}\right\}$ are separated by Lemma $\left.5.5 \mathrm{i}\right)$, so that we use Remark 5.6 again. Statement $\left(S_{2}\right)$ is an immediate consequence of Lemma $6.4 \mathrm{ii}) . \bar{C}_{j}^{(1)}$ is separated from $\bar{C}_{j, l}^{(22)}$ by (6.4), and moreover it is separated from $\bar{C}_{l}^{(3)}$. In fact, as $\bar{C}_{l}^{(3)} \subseteq \Pi_{n_{2}, n_{1}}^{-1}\left(\mathcal{A}_{2, n_{1}}\right)$, then the elements of $\bar{C}_{l}^{(3)}$ have the form $\left(i_{1}, \ldots, i_{n_{2}}\right)$, and $\left(i_{1}, \ldots, i_{n_{2}-1}\right) \neq j_{\left(n_{2}-1\right)}$, and we use Lemma 5.5 i). To prove $\left(S_{4}\right)$, note that in the opposite case, we have $\bar{\varpi}_{s} \sim \varpi^{\prime} \in \bar{C}_{l}^{3)}$ for some $s \leq \tilde{s}$, thus the path $\left(\bar{\varpi}_{0}, \ldots, \bar{\varpi}_{s}, \varpi^{\prime}\right)$ connects $j_{\left(n_{2}\right)}$ to $\varpi^{\prime} \in \bar{C}_{l}^{(3)}$ in $\bar{C}_{j, l}^{(2)}$, and its length is $s+1 \leq \tilde{s}+1<\bar{m}$, and this contradicts the assumption that the original path $\left(\bar{\varpi}_{0}, \ldots, \bar{\varpi}_{\bar{m}}\right)$ was of minimum length with such properties.

In order to evaluate the effective resistances between two points, we have to investigate the intersections of the $V$-copies of the previously defined sets. When $j \in \widetilde{B}_{l}^{\prime}$, let us define

$$
\begin{gathered}
M_{j, l}=V\left(\bar{C}_{j}^{(1)}\right) \cap V\left(\bar{C}_{j, l}^{(21)}\right), \\
M_{j, l}^{\prime}=V\left(\bar{C}_{j, l}^{(21)}\right) \cap V\left(\bar{C}_{j, l}^{(22)}\right), \\
M_{j, l}^{\prime \prime}=V\left(\bar{C}_{j, l}^{(22)}\right) \cap V\left(\bar{C}_{l}^{(3)}\right), \\
M_{(j, n)}=V\left(\left\{j_{(n)}\right\}\right) \cap V\left(\Pi_{n, n_{2}}^{-1}\left(j_{\left(n_{2}\right)}\right) \backslash\left\{j_{(n)}\right\}\right), \quad j=1, \ldots, N, n>n_{2} .
\end{gathered}
$$

Lemma 6.9. For every $n \geq n_{2}$ we have

$$
\begin{gathered}
M_{j, l}=V\left(\bar{G}_{j, n}^{(1)}\right) \cap V\left(\bar{G}_{j, l, n}^{(21)}\right), \\
M_{j, l}^{\prime}=V\left(\bar{G}_{j, l, n}^{(21)}\right) \cap V\left(\bar{G}_{j, l, n}^{(22)}\right),
\end{gathered}
$$




$$
M_{j, l}^{\prime \prime}=V\left(\bar{G}_{j, l, n}^{(22)}\right) \cap V\left(\bar{G}_{l, n}^{(3)}\right) .
$$

Moreover, the sets $M_{j, l}, M_{j, l}^{\prime} M_{j, l}^{\prime \prime}, M_{(j, n)}, n>n_{2}$, are nonempty.

Proof. The first statement immediately follows from Lemma 5.4 ii and Lemma $6.2 \mathrm{i}$ ). To prove that the sets $M_{j, l}, M_{j, l}^{\prime}, M_{j, l}^{\prime \prime}$ are nonempty, it suffices to observe that $\left(\bar{\varpi}_{0}, \ldots, \bar{\varpi}_{\bar{m}}\right)$ is a path, and $\bar{\varpi}_{0} \in \bar{C}_{j}^{(1)}, \bar{\varpi}_{1}, \bar{\varpi}_{\tilde{s}} \in \bar{C}_{j, l}^{(21)}, \bar{\varpi}_{\tilde{s}+1}, \bar{\varpi}_{\bar{m}-1} \in \bar{C}_{j, l}^{(22)}, \bar{\varpi}_{\bar{m}} \in C_{l}^{(3)}$. Finally, to prove that $M_{(j, n)} \neq \varnothing$, note that, by the connectedness of $\mathcal{F}_{n}$ there exists $\varpi \in \mathcal{A}_{3, n}$, $\varpi \neq j_{(n)}$ such that $V_{j_{(n)}} \cap V_{\varpi} \neq \varnothing$. By Corollary 5.5 , we have $\varpi \in \Pi_{n, n_{2}}^{-1}\left(j_{\left(n_{2}\right)}\right)$, so that by definition, $M_{(j, n)} \neq \varnothing$.

Lemma 6.10. For every $n>n_{2}$, the sets $\left\{P_{j}\right\}, M_{j, l}, M_{j, l}^{\prime}, M_{j, l}^{\prime \prime}$ with all $j, l$ such that $j \in \widetilde{B}_{l}^{\prime}$, and $M_{(j, n)}$ with $j=1, \ldots, N$, are mutually disjoint.

Proof. The sets $M_{j, l}, M_{j, l}^{\prime}, M_{j, l}^{\prime \prime}$ are mutually disjoint by Lemma 6.8 and formulas (6.9), (6.10), (6.11). Also, they are disjoint from the sets $\left\{P_{j}\right\}, M_{(j, n)}$ by Lemma 5.5. By Lemmas 5.1 and 5.5 again, the sets $\left\{P_{j}\right\}, M_{(j, n)}$ are mutually disjoint.

We will now define new sets, useful in the sequel, defined as the union of previously defined sets. Let

$$
\begin{gathered}
\bar{C}_{j, l}^{(17)}=\bar{C}_{j}^{(1)} \cup \bar{C}_{j, l}^{(7)} \\
\bar{C}_{j, l}^{(1217)}=\bar{C}_{j}^{(1)} \cup \bar{C}_{j, l}^{(21)} \cup \bar{C}_{j, l}^{(7)} \\
\bar{C}_{j, l}^{(121)}:=\bar{C}_{j}^{(1)} \cup \bar{C}_{j, l}^{(21)} \text { if } j \in \widetilde{B}_{l}^{\prime}, \quad \bar{C}_{j}^{(121)}=\bigcup_{l: j \in \widetilde{B}_{l}^{\prime}} \bar{C}_{j, l}^{(121)}
\end{gathered}
$$

and $\bar{G}_{j, l, n}^{(17)}, \bar{C}_{j, l, n}^{(1217)}, \bar{G}_{j, l, n}^{(121)}, \bar{G}_{j, n}^{(121)}$ as usual.

Lemma 6.11. The sets $\bar{C}_{j, l}^{(17)}, \bar{C}_{j, l}^{(1217)}, \bar{C}_{j, l}^{(121)}, \bar{C}_{j}^{(121)}, \bar{C}_{j, l}^{(121)} \cup \bar{C}_{j, l^{\prime}}^{(121)}$, and consequently, $\bar{G}_{j, l, n}^{(17)}, \bar{G}_{j, l, n}^{(1217)}, \bar{G}_{j, l, n}^{(121)}, \bar{G}_{j, n}^{(121)}, \bar{G}_{j, l, n}^{(121)} \cup \bar{G}_{j, l^{\prime}, n}^{(121)}$ are connected.

Proof. It suffices to observe that $\bar{C}_{j}^{(1)} \cup \bar{C}_{j, l}^{(21)}$ is a path, and that, by definition, every point of $\bar{C}_{j, l}^{(7)}$ is connected to $j_{\left(n_{2}\right)} \in \bar{C}_{j}^{(1)}$ by a path in $\bar{C}_{j, l}^{(7)}$. Thus, in any of the sets $\bar{C}_{j, l}^{(17)}$, $\bar{C}_{j, l}^{(1217)}, \bar{C}_{j, l}^{(121)}, \bar{C}_{j}^{(121)}$, every point is connected to $j_{\left(n_{2}\right)}$ by a path contained in the set.

Remark 6.12. Note that, by Remark 6.7, when $j \in \widetilde{B}_{l}^{\prime}$, then $\bar{G}_{j, l, n}^{(7)}$ is separated from $\bar{G}_{n}^{(5)} \backslash \bar{G}_{j, l, n}^{(1217)}$. Therefore, in view also of Lemma 6.8 , if $\varpi \in \bar{G}_{j, l, n}^{(1217)}, \varpi^{\prime} \in \bar{G}_{n}^{(5)} \backslash \bar{G}_{j, l, n}^{(1217)}$ and $\varpi \sim \varpi^{\prime}$, then, $\varpi \in \bar{G}_{j, l, n}^{(21)}, \varpi^{\prime} \in \bar{G}_{j, l, n}^{(22)}$; thus $V\left(\bar{G}_{j, l, n}^{(1217)}\right) \cap V\left(\bar{G}_{n}^{(5)} \backslash \bar{G}_{j, l, n}^{(1217)}\right) \subseteq M_{j, l}^{\prime}$. Using Lemma 6.8 again, we can prove similarly that, if $\varpi \in \bar{G}_{j, l, n}^{(121)}, j \in \widetilde{B}_{l}^{\prime}, \varpi^{\prime} \in \bar{G}_{n}^{(5)} \backslash \bar{G}_{j, n}^{(121)}$ and $\varpi \sim \varpi^{\prime}$, then we have $\varpi \in \bar{G}_{j, l, n}^{(21)}, \varpi^{\prime} \in \bar{G}_{j, l, n}^{(22)}$; thus $V\left(\bar{G}_{j, l, n}^{(121)}\right) \cap V\left(\bar{G}_{n}^{(5)} \backslash \bar{G}_{j, n}^{(121)}\right) \subseteq M_{j, l}^{\prime}$. 


\section{Several Lemmas.}

The present Section is devoted to prove several preparatory results for the proof of the main Theorem. First of all I will introduce an element $\bar{q}$ of $\widetilde{\mathcal{Q}}$, that in the proof of main Theorem plays the role that $1_{J}$ played in Section 4 . In this more general case, we need a form that is less symmetric than $\bar{I}\left(1_{J}\right)$, in the sense that is symmetric only restricted to a single block. Recall that the graph $\bar{J}$ on $\{1, \ldots, N\}$ is defined by

$$
\bar{J}=\left\{\left\{j_{1}, j_{2}\right\}: j_{1}, j_{2}=1, \ldots, N, j_{1} \neq j_{2}, \exists l=1, \ldots, h^{\prime}: j_{1}, j_{2} \in \widetilde{B}_{l}^{\prime}\right\} \subseteq J .
$$

In view of $\left(6.3^{\prime}\right)$, it is simple to see that $\bar{J}$ on $\{1, \ldots, N\}$ is connected. Thus $\bar{q} \in \widetilde{\mathcal{Q}}$ defined by $\bar{q}=\chi_{\bar{J}}$, in fact belongs to $\widetilde{\mathcal{Q}}$, since $\mathcal{G} r(\bar{q})=\bar{J}$. In order to investigate the properties of $\bar{q}$, I will now define a set that is a sort of analog of $\bar{C}_{j, l}^{(7)}$ in $\{1, \ldots, N\}$. Namely, if $j \in \widetilde{B}_{l}^{\prime}$, we denote by $\widetilde{C}_{j, l}^{(7)}$ the set of $j^{\prime} \in\{1, \ldots, N\} \backslash \widetilde{B}_{l}^{\prime} \bar{J}$-connected to $j$ in $\{1, \ldots, N\} \backslash \widetilde{B}_{l}^{\prime}$.

\section{Lemma 7.1.}

i) For every $j \in \widetilde{B}_{l}^{\prime}, j^{\prime}=1, \ldots, N$, we have $j^{\prime} \in \widetilde{C}_{j, l}^{(7)} \Longleftrightarrow j_{\left(n_{2}\right)}^{\prime} \in \bar{C}_{j, l}^{(7)}$,

ii) The set $\{1, \ldots, N\} \backslash \widetilde{B}_{l}^{\prime}$ is the union of the mutually disjoint sets $\widetilde{C}_{j, l}^{(7)}, j \in \widetilde{B}_{l}^{\prime}$.

iii) if $j_{1} \in \widetilde{C}_{j, l}^{(7)}, j_{2} \in \widetilde{C}_{j^{\prime}, l}^{(7)}$, with $j, j^{\prime} \in \widetilde{B}_{l}^{\prime}, j \neq j^{\prime}$, then $\left\{j_{1}, j_{2}\right\} \notin \bar{J}$.

Proof. We first prove the " $\Rightarrow$ " part in i). Suppose $j^{\prime} \in \widetilde{C}_{j, l}^{(7)}$. Then, there exists a $\bar{J}$ path $\left(j_{0}, \ldots, j_{m}\right)$ in $\{1, \ldots, N\} \backslash \widetilde{B}_{l}^{\prime}$ connecting $j^{\prime}$ to $j$, thus two consecutive vertices of the path $j_{s-1}, j_{s}$ belong to a common $\widetilde{B}_{u(s)}^{\prime}$ for every $s=1, . ., m$ and of course $u(s) \neq l$ as $j_{s-1} \notin \widetilde{B}_{l}^{\prime}$. Pick a point $\bar{\varpi} \in \bar{C}_{u(s)}^{(3)}$. By Lemma 6.5 there exist a $\mathcal{G}$-path in $\bar{C}_{j_{s-1}, u(s)}^{(8)}$ connecting $\left(j_{s-1}\right)_{\left(n_{2}\right)}$ to $\bar{\varpi}$ and a $\mathcal{G}$-path in $\bar{C}_{j_{s}, u(s)}^{(8)}$ connecting $\left(j_{s}\right)_{\left(n_{2}\right)}$ to $\bar{\varpi}$. Therefore, there exists a path connecting $\left(j_{s-1}\right)_{\left(n_{2}\right)}$ to $\left(j_{s}\right)_{\left(n_{2}\right)}$ in $\mathcal{A}_{2, n_{2}} \cap\left(\bar{C}^{(5)} \backslash \bar{C}_{l}^{(4)}\right)$. As a consequence, $j_{\left(n_{2}\right)}^{\prime}$ is connected to $j_{\left(n_{2}\right)}$ in $\bar{C}^{(5)} \backslash \bar{C}_{l}^{(4)}$, thus $j_{\left(n_{2}\right)}^{\prime} \in \bar{C}_{j, l}^{(7)}$ and the " $\Rightarrow$ " part in i) is proved. By Lemma 6.6 the sets $\widetilde{C}_{j, l}^{(7)}, j \in \widetilde{B}_{l}^{\prime}$ are mutually disjoint. Next, we prove that

$$
\{1, \ldots, N\} \backslash \widetilde{B}_{l}^{\prime}=\bigcup_{j \in \widetilde{B}_{l}^{\prime}} \widetilde{C}_{j, l}^{(7)}
$$

It clearly suffices to prove the inclusion $\subseteq$. If $j^{\prime} \in\{1, \ldots, N\} \backslash \widetilde{B}_{l}^{\prime}$, there exists a $\bar{J}$-path connecting $j^{\prime}$ to some point in $\widetilde{B}_{l}^{\prime}$, and suppose $j$ is the first vertex in the path belonging to $\widetilde{B}_{l}^{\prime}$. Then $j^{\prime} \in \widetilde{C}_{j, l}^{(7)}$ and (7.1) follows, thus ii) is proved. Next, we prove the " $\Leftarrow$ " part in i). Suppose $j \in \widetilde{B}_{l}^{\prime}, j^{\prime}=1, \ldots, N$ and $j_{\left(n_{2}\right)}^{\prime} \in \bar{C}_{j, l}^{(7)}$. Then $j^{\prime} \in\{1, \ldots, N\} \backslash \widetilde{B}_{l}^{\prime}$, so that $j^{\prime} \in \widetilde{C}_{j^{\prime \prime}, l}^{(7)}$ for some $j^{\prime \prime} \in \widetilde{B}_{l}^{\prime}$. Thus, $j_{\left(n_{2}\right)}^{\prime} \in \bar{C}_{j^{\prime \prime}, l}^{(7)}$, so that, by Lemma 6.5 again, $j^{\prime \prime}=j$ and $\Leftarrow$ in i) is proved. Finally, iii) immediately follows from ii).

We now are going to evaluate $\bar{R}(\bar{q})$. To this aim, we need some preliminary considerations. First, we will evaluate the resistance between two different $P_{j}$ lying in the same block. 
The evaluation of $\bar{R}(\bar{q})$ restricted to a single $n$-block is simple and is essentially the same problem as the evaluation of $\widehat{R}\left(1_{j}\right)$ in the case of C.I.F.T.. However, we have to prove that such effective resistance (along $\bar{C}_{l}^{(4)}$ ) is the same as that along $\bar{C}^{(5)}$. Lemmas 7.2 and 7.4 are the tools to obtain this, while Lemma 7.3 will lead us to evaluate $\bar{R}(q)$ between points in different blocks. We say that a subset $W^{\prime}$ of a graph $(W, G)$ is normally disconnecting (in $W$ ) if every $Q \in W \backslash W^{\prime}$ is $G$ connected to at most one point of $W^{\prime}$ in $W \backslash W^{\prime}$.

Lemma 7.2. If $V^{\prime} \subseteq V, V^{\prime}=\left\{P_{j}: j \in C\right\}$, \# $\left(V^{\prime}\right) \geq 2, q \in \mathcal{Q}$, and $C$ is $\mathcal{G} r(q)$-normally disconnecting. Putting $q^{\prime}:=\left.q\right|_{J(C)}$ then for every $P_{j_{1}}, P_{j_{2}} \in V^{\prime}, j_{1} \neq j_{2}$

i) $\left.\bar{I}(q)\right|_{V^{\prime}}=\bar{I}\left(q^{\prime}\right)$,

ii) $\bar{R}(q)_{\left\{j_{1}, j_{2}\right\}}=\bar{R}\left(q^{\prime}\right)_{\left\{j_{1}, j_{2}\right\}}$.

Proof. The statement is well-known so that I omit the proof. The idea is that every function on $V^{\prime}$ can be extended to a function on $V$, constant on every component of $V \backslash V^{\prime}$.

Lemma 7.3. Suppose $q \in \mathcal{Q}, V=V^{\prime} \cup V^{\prime \prime}$, and $V^{\prime} \cap V^{\prime \prime}=\left\{P_{\bar{j}}\right\}, V^{\prime}=\left\{P_{j}: j \in C^{\prime}\right\}$, $V^{\prime \prime}=\left\{P_{j}: j \in C^{\prime \prime}\right\}$ with $C^{\prime}$ and $C^{\prime \prime}$ subsets of $\{1, \ldots, N\}$. Suppose $\left\{j_{1}, j_{2}\right\} \notin \mathcal{G} r(q)$ for every $j_{1} \in C^{\prime} \backslash\{\bar{j}\}, j_{2} \in C^{\prime \prime} \backslash\{\bar{j}\}$. Then for every $P_{j_{1}} \in V^{\prime} \backslash\left\{P_{\bar{j}}\right\}, P_{j_{2}} \in V^{\prime \prime} \backslash\left\{P_{\bar{j}}\right\}$

i) $\bar{R}(q)_{\left\{j_{1}, \bar{j}\right\}}=\bar{R}\left(\left.q\right|_{J\left(C^{\prime}\right)}\right)_{\left\{j_{1}, \bar{j}\right\}}, \quad \bar{R}(q)_{\left\{j_{2}, \bar{j}\right\}}=\bar{R}\left(\left.q\right|_{J\left(C^{\prime \prime}\right)}\right)_{\left\{j_{2}, \bar{j}\right\}}$

ii) $\bar{R}(q)_{\left\{j_{1}, j_{2}\right\}}=\bar{R}(q)_{\left\{j_{1}, \bar{j}\right\}}+\bar{R}(q)_{\left\{j_{2}, \bar{j}\right\}}$.

Proof. i) follows from Lemma 7.1, and ii) is a case of the well-known resistances in series principle.

Lemma 7.4. For every $l=1, . ., h^{\prime}$, the sets $\widetilde{B}_{l}^{\prime}$ and $\bigcup_{h=1}^{l} \widetilde{B}_{h}^{\prime}$ are $\bar{J}$-normally disconnecting.

Proof. The set $\widetilde{B}_{l}^{\prime}$ is normally disconnecting by Lemma 7.1. We prove that $B:=\bigcup_{h=1}^{l} \widetilde{B}_{h}^{\prime}$ is normally disconnecting too. Suppose there is a point in $\{1, \ldots, N\} \backslash B$ connected in $\{1, \ldots, N\} \backslash B$ to two different points $j$ and $j^{\prime}$ of $B$. Then, $j$ and $j^{\prime}$ are connected by a simple path $\left(j_{0}, \ldots, j_{m}\right)$ with $j_{s} \in\{1, \ldots, N\} \backslash B$ for $s=1, \ldots, m-1, m>1$. Let $\bar{l}$ be the maximum $h$ such that $\widetilde{B}_{h}^{\prime}$ contains at least two different $j_{s}$, namely $j_{s_{1}}$ and $j_{s_{2}}$. Suppose $s_{1}, s_{2}$ are respectively the minimum and the maximum of the set of the indices $s$ such that $j_{s} \in \widetilde{B}_{\bar{l}}^{\prime}$. As $j_{1} \notin B$, and by hypothesis $j_{0}, j_{1}$ belong to a common $\widetilde{B}_{s}^{\prime}$ we have $s>l$, thus $\bar{l}>l$. In any case, we have $j_{s_{1}}, j_{s_{2}} \in \widetilde{B}_{\bar{l}}^{\prime} \cap\left(\bigcup_{m=1}^{\bar{l}-1} \widetilde{B}_{m}^{\prime}\right)$ and this contradicts $\left(6.3^{\prime}\right)$.

In order to completely evaluate $\bar{R}(\bar{q})$, we have still to discuss what happens between points in different blocks. To this aim, I will now introduce to following notion.

We say that $\left(j_{1}, j_{2}\right)$ is a $\bar{l}$-pair if $j_{1} \in\left(\bigcup_{l=1}^{\bar{l}} \widetilde{B}_{l}^{\prime}\right) \backslash \widetilde{B}_{\bar{l}+1}^{\prime}, j_{2} \in \widetilde{B}_{\bar{l}+1}^{\prime} \backslash\left(\bigcup_{l=1}^{\bar{l}} \widetilde{B}_{l}^{\prime}\right)$.

\section{Lemma 7.5.}


i) If $j_{1}, j_{2} \in \bigcup_{l=1}^{\bar{l}+1} \widetilde{B}_{l}^{\prime}, j_{1} \neq j_{2}$, then precisely one of the following occurs

a) there exists $l=1, \ldots, \bar{l}+1$ such that $j_{1}, j_{2} \in \widetilde{B}_{l}^{\prime}$

b) there exists $l^{\prime}=1, \ldots, \bar{l}$ such that either $\left(j_{1}, j_{2}\right)$ or $\left(j_{2}, j_{1}\right)$ is an $l^{\prime}$-pair.

ii) If $\left(j_{1}, j_{2}\right)$ is a $\bar{l}$-pair, then $j_{1}, j_{2}$ and $\tilde{j}_{\bar{l}}$ are mutually different.

Proof. If there exists no $l=1, \ldots, \bar{l}+1$ such that $j_{1}, j_{2} \in \widetilde{B}_{l}^{\prime}$, then, let $l_{1}$ be the minimum $l$ such that $j_{1} \in \widetilde{B}_{l}^{\prime}$ and let $l_{2}$ be the minimum $l$ such that $j_{2} \in \widetilde{B}_{l}^{\prime}$. We have $l_{1} \neq l_{2}$ and, if for example $l_{1}<l_{2}$, then $\left(j_{1}, j_{2}\right)$ is a $\left(l_{2}-1\right)$-pair. Moreover, if both a) and b) hold, by definition, we have $l^{\prime}+1<l$, but in such a case, $j_{1}, j_{2} \in\left(\bigcup_{s=1}^{l-1} \widetilde{B}_{s}^{\prime}\right) \cup \widetilde{B}_{l}^{\prime}$, and this contradicts $\left(6.3^{\prime}\right)$, and i) is proved, and ii) is an immediate consequence of the definition of an $l$-pair and $\left(6.3^{\prime}\right)$.

Now, putting $\widetilde{\bar{R}}:=\bar{R}(\bar{q})$, we are ready to find $\widetilde{\widetilde{R}}$ recursively:

Lemma 7.6. If $\left\{j_{1}, j_{2}\right\} \in J$, then

$$
\widetilde{\bar{R}}_{\left\{j_{1}, j_{2}\right\}}= \begin{cases}\frac{2}{\#\left(\widetilde{B}_{l}^{\prime}\right)} & \text { if } j_{1}, j_{2} \in \widetilde{B}_{l}^{\prime}, \\ \widetilde{\bar{R}}_{\left\{\tilde{j}_{l}, j_{1}\right\}}+\widetilde{\bar{R}}_{\left\{\tilde{j}_{l}, j_{2}\right\}} & \text { if }\left(j_{1}, j_{2}\right) \text { is l-pair } .\end{cases}
$$

Proof. Suppose $j_{1}, j_{2} \in \widetilde{B}_{l}^{\prime}$. By Lemma 7.2 , we have

$$
\widetilde{\bar{R}}_{\left\{j_{1}, j_{2}\right\}}=\bar{R}\left(\left.\bar{q}\right|_{J\left(\widetilde{B}_{l}^{\prime}\right)}\right)_{\left\{j_{1}, j_{2}\right\}}
$$

but $\left.\bar{q}\right|_{J\left(\widetilde{B}_{l}^{\prime}\right)} \equiv 1$, thus the minimum in the definition of $(1 / \bar{R})\left(\left.\bar{q}\right|_{J\left(\widetilde{B}_{l}^{\prime}\right)}\right)$ is attained at the function $\bar{v}$ that attains the value $\frac{1}{2}$ at all points different from $j_{1}$ and $j_{2}$, and $\bar{I}\left(\left.\bar{q}\right|_{J\left(\widetilde{B}_{l}^{\prime}\right)}\right)(\bar{v})=$ $\frac{\#\left(\widetilde{B}_{l}^{\prime}\right)}{2}$, so that the lemma is proved in this case. Putting $B=\bigcup_{s=1}^{l+1} \widetilde{B}_{s}^{\prime}$, we have

$$
\widetilde{\bar{R}}_{\left\{\tilde{j}_{l}, j_{1}\right\}}=\bar{R}\left(\left.\bar{q}\right|_{J(B)}\right)_{\left\{\tilde{j}_{l}, j_{1}\right\}}, \quad \widetilde{\bar{R}}_{\left\{\tilde{j}_{l}, j_{2}\right\}}=\bar{R}\left(\left.\bar{q}\right|_{J(B)}\right)_{\left\{\tilde{j}_{l}, j_{2}\right\}}, \quad \widetilde{\bar{R}}_{\left\{j_{1}, j_{2}\right\}}=\bar{R}\left(\left.\bar{q}\right|_{J(B)}\right)_{\left\{j_{1}, j_{2}\right\}},
$$

by Lemmas 7.2 and 7.4. Thus, the formula when $\left(j_{1}, j_{2}\right)$ is $l$-pair follows from Lemma 7.3 where we put $V=\left\{P_{j}: j \in B\right\}, C^{\prime}=\bigcup_{s=1}^{l} \widetilde{B}_{s}^{\prime}, C^{\prime \prime}=\widetilde{B}_{l+1}^{\prime}$.

Let

$$
\widetilde{K}=\left\{q \in \mathbb{R}^{J}: q_{d} \in[1-\varsigma, 1+\varsigma] \forall d \in \bar{J}, q_{d} \in[0, \varsigma] \forall d \in J \backslash \bar{J}\right\} \subseteq \widetilde{\mathcal{Q}}
$$


for positive $\varsigma<1$, which we will fix in the sequel. Note that $\widetilde{K}$ is a nonempty compact convex subset of $\mathbb{R}^{J}$. I will prove that, for a suitable $n$, a suitable continuous map $\sigma: \widetilde{K} \rightarrow$ $W$, the map $q \mapsto \widehat{\Lambda}_{\sigma(q)}(q)$ sends $\widetilde{K}$ into itself, thus it has a fixed point.

Lemma 7.7. There exists a neighbourhood $\bar{U}$ of $\widetilde{\bar{R}}$ such that, if $q \in \mathcal{Q}$ and $\bar{R}(q) \in \bar{U}$, then $q \in \widetilde{K}$.

Proof. By Lemma 2.8 iv), there exists $U$ open neighbourhood of $\widetilde{\bar{R}}$ such that $\bar{R}^{-1}(U)$ is a bounded subset of $\widetilde{\mathcal{Q}}$. Thus, by Lemmas 2.8 iii), $\bar{R}$ is a homemomorphism from $\bar{R}^{-1}(U)$ onto

$$
\bar{R}\left(\bar{R}^{-1}(U)\right)=: V \subseteq U
$$

Hence, as $\bar{R}^{-1}(\widetilde{\bar{R}})=\bar{q}$ and $\widetilde{K}$ is a neighbourhood of $\bar{q}$ in $\widetilde{\mathcal{Q}}$, there exists $\widetilde{U}$ open neighbourhood of $\widetilde{R}$ in $V$ such that if $y \in \widetilde{U}$, then $\bar{R}^{-1}(y) \in \widetilde{K}$. We have $\widetilde{U}=\bar{U} \cap V$ for some $\bar{U}$ open set in $\mathbb{R}^{J}$ contained in $U$. We easily see that $\bar{U}$ satisfies the Corollary.

In the sequel, we will denote by $\widehat{\Lambda}_{r ; n}$ the operator $\widehat{\Lambda}_{r}$ on $\mathcal{F}_{n}$ for $n>1$ when there is possibility of confusion, for example $\widehat{\Lambda}_{1 ; n}$. Usually, we can see that $\widehat{\Lambda}_{r}$ is defined on $\mathcal{F}_{n}$ on the basis that $r \in] 0,+\infty\left[\mathcal{A}_{3, n}\right.$. A well-known result is that

$$
\widehat{\Lambda}_{1 ; n}=\widehat{\Lambda}_{1}^{n}:=\widehat{\Lambda}_{1 ; 1}^{n} \text {. }
$$

We will now investigate some properties of $\widehat{\Lambda}_{r}$ useful for the sequel. First, we prove that $\widehat{\Lambda}_{1}$ goes to 0 when $n$ goes to infinity, and this fact will play an essential role in the sequel as, for example, will lead us to prove that the contribution of the cells on the residual set $\bar{C}^{(6)}$ negligible for $n$ large enough.

Lemma 7.8. $\widehat{\Lambda}_{1}^{n}(q)_{\left\{j_{1}, j_{2}\right\}} \underset{n \rightarrow+\infty}{\longrightarrow} 0$ for every $q \in \mathcal{Q}$ and $\left\{j_{1}, j_{2}\right\} \in J$.

Proof. For every $q \in \mathcal{Q}, n \in \mathbb{N}$ and $j=1, \ldots, N$, let

$$
\tilde{c}_{j, n}(q)=\sum_{j^{\prime} \neq j} \widehat{\Lambda}_{1}^{n}(q)_{\left\{j, j^{\prime}\right\}}, \quad \tilde{\tilde{c}}_{n}(q)=\sum_{j=1}^{N} \tilde{c}_{j, n}(q) .
$$

Of course $\tilde{c}_{j, n}\left(\widehat{\Lambda}_{1}^{m}(q)\right)=\tilde{c}_{j, n+m}(q)$. Clearly,

$$
\tilde{c}_{j, n}(q)=\bar{I}\left(\widehat{\Lambda}_{1}^{n}(q)\right)\left(\chi_{\left\{P_{j}\right\}}\right) .
$$

We now prove

$$
\begin{gathered}
\tilde{c}_{j, 1}(q) \leq \tilde{c}_{j, 0}(q) \\
\tilde{c}_{j, 1}(q)<\tilde{c}_{j, 0}(q) \quad \text { if } \tilde{c}_{j, 0}(q)>0 .
\end{gathered}
$$

In order to prove $(7.3)$ and $\left(7.3^{\prime}\right)$, for every $t \in \mathbb{R}$, define $v_{t} \in \mathbb{R}^{V^{(1)}}$ by 


$$
v_{t}=\chi_{\left\{P_{j}\right\}}+t \chi_{V^{(1)} \backslash V^{(0)}}
$$

thus we have

$$
\begin{gathered}
\tilde{c}_{j, 1}(q)=\Lambda_{1}(\bar{I}(q))\left(\chi_{\left\{P_{j}\right\}}\right) \leq S_{1}^{\prime \prime}(\bar{I}(q))\left(v_{t}\right)=\bar{I}(q)\left(v_{t} \circ \psi_{j}\right)+\sum_{j^{\prime}=1, \ldots, N, j^{\prime} \neq j} \bar{I}(q)\left(v_{t} \circ \psi_{j^{\prime}}\right) \\
=\bar{I}(q)\left(t+(1-t) \chi_{\left\{P_{j}\right\}}\right)+\sum_{j^{\prime}=1, \ldots, N, j^{\prime} \neq j} \bar{I}(q)\left(t-t \chi_{\left\{P_{j^{\prime}}\right\}}\right) \\
=(1-t)^{2} \bar{I}(q)\left(\chi_{\left\{P_{j}\right\}}\right)+t^{2} \sum_{j^{\prime}=1, \ldots, N, j^{\prime} \neq j} \bar{I}(q)\left(\chi_{\left\{P_{j^{\prime}}\right\}}\right)=(1-t)^{2} \tilde{c}_{j, 0}(q)+t^{2} \sum_{j^{\prime}=1, \ldots, N, j^{\prime} \neq j} \tilde{c}_{j^{\prime}, 0}(q)
\end{gathered}
$$

for every $t \in \mathbb{R}$. Letting $\gamma$ to be the function defined in the last row, we have $\gamma(0)=\tilde{c}_{j, 0}(q)$ and (7.3) follows at once. If moreover $\tilde{c}_{j, 0}(q)>0$, then $\gamma^{\prime}(0)<0$, and $\left(7.3^{\prime}\right)$ follows. Note that the function $\tilde{c}_{j, 0}, \tilde{c}_{j, 1}$ and $\tilde{\tilde{c}}_{o}$ are continuous. Thus the set

$$
A_{\alpha, K}:=\left\{q \in \mathcal{Q}: \tilde{c}_{j, 0}(q) \geq \alpha, \tilde{\tilde{c}}_{0}(q) \leq K\right\}
$$

is compact for every positive $\alpha, K$. Thus, if $q \in A_{\alpha, K}$, then

$$
\tilde{c}_{j, 1}(q) \leq \tilde{c}_{j, 0}(q)-\beta
$$

where $\beta$ is a positive constant (depending on $\alpha$ and $K$ ). Therefore, given $K>\tilde{\tilde{c}}_{0}(q)$, we have $K>\tilde{\tilde{c}}_{n}(q)$ for every $n$, by (7.3). Suppose now given $\alpha>0$. Then, if $\tilde{c}_{j, n}(q) \geq \alpha$ for every $n$ we would have $\tilde{c}_{j, n}(q) \leq \tilde{c}_{j, 0}(q)-n \beta$ by (7.4), a contradiction. Thus, as by (7.3) the sequence $\tilde{c}_{j, n}(q)$ is decreasing, then $\tilde{c}_{j, n}(q)<\alpha$ for sufficiently large $n$. Therefore, $\tilde{c}_{j, n}(q) \underset{n \rightarrow+\infty}{\longrightarrow} 0$ and the statement of the Lemma easily follows.

Corollary 7.9. $\left|\widehat{\Lambda}_{1 ; n}(q)\right|=\left|\widehat{\Lambda}_{1}^{n}(q)\right| \underset{n \rightarrow+\infty}{\longrightarrow} 0$, uniformly on $B_{a}$ for every $a>0$.

Proof. If $q \in B_{a}$, then $\bar{I}(q) \leq|q| \bar{I}\left(1_{J}\right) \leq a \bar{I}\left(1_{J}\right)$, therefore $\bar{I}\left(\widehat{\Lambda}_{1}^{n}(q)\right) \leq a \bar{I}\left(\widehat{\Lambda}_{1}^{n}\left(1_{J}\right)\right)$. Thus,

$$
\widehat{\Lambda}_{1}^{n}(q)_{\left\{j_{1}, j_{2}\right\}} \leq \frac{1}{4} \bar{I}\left(\widehat{\Lambda}_{1}^{n}(q)\right)\left(\chi_{P_{j_{1}}}-\chi_{P_{j_{2}}}\right) \leq \frac{1}{4} a \bar{I}\left(\widehat{\Lambda}_{1}^{n}\left(1_{J}\right)\right)\left(\chi_{P_{j_{1}}}-\chi_{P_{j_{2}}}\right)
$$

and we conclude by Lemma 7.8, and (7.2).

The final results of this Section are generalizations of Lemma 3.3. In fact, Lemma 3.3 can be seen as a particular case of Lemma 7.10 when $n=0$.

Lemma 7.10. Let $\mathcal{B}$ be a nonempty subset of $\mathcal{A}_{3, n}$ and let $\left.r^{\prime} \in\right] 0,+\infty\left[{ }^{\mathcal{B}}, r_{\varpi}^{\prime \prime} \in\right] 0,+\infty\left[\mathcal{A}_{3, m}\right.$ for every $\varpi \in \mathcal{B}$. Let $q_{\varpi} \in \widetilde{\mathcal{Q}}$ for every $\varpi \in \mathcal{B}$, let $r \in \mathbb{R}^{\mathcal{B} \times \mathcal{A}_{3, m}}$ be defined by $r\left(\varpi, \varpi^{\prime \prime}\right)=$ $r^{\prime}(\varpi) r_{\varpi}^{\prime \prime}\left(\varpi^{\prime \prime}\right)$. Let $M_{1}, M_{2}$ be nonempty disjoint subsets of $V(\mathcal{B})$. Then

$$
\widehat{R}\left(S_{r^{\prime}, \varpi, \mathcal{B}}\left(\widehat{\Lambda}_{r_{\varpi}^{\prime \prime}}\left(q_{\varpi}\right)\right)\right)_{\left\{M_{1}, M_{2}\right\}}=\widehat{R}\left(S_{r,\left(\varpi, \varpi^{\prime \prime}\right), \mathcal{B} \times \mathcal{A}_{3, m}}\left(q_{\varpi}\right)\right)_{\left\{M_{1}, M_{2}\right\}} .
$$


Proof. Note that by Lemma 5.4, $V(B) \subseteq V\left(\Pi_{n+m, n}^{-1}(B)\right)=V\left(B \times \mathcal{A}_{3, m}\right)$, so that $M_{1}$ are also nonempty disjoint subsets of $V\left(B \times \mathcal{A}_{3, m}\right)$. As for every $v \in \mathbb{R}^{V^{(m)}}$ we have

$$
S_{r_{\varpi}^{\prime \prime}}^{\prime \prime}\left(q_{\varpi}\right)(v) \geq \Lambda_{r_{\varpi}^{\prime \prime}}\left(\bar{I}\left(q_{\varpi}\right)\right)\left(\left.v\right|_{V^{(0)}}\right)=\bar{I}\left(\widehat{\Lambda}_{r_{\varpi}^{\prime \prime}}\left(q_{\varpi}\right)\right)\left(\left.v\right|_{V^{(0)}}\right)
$$

then for every $w \in \mathbb{R}^{V\left(\mathcal{B} \times \mathcal{A}_{3, m}\right)}$ we have,

$$
\begin{aligned}
& S_{r,\left(\varpi, \varpi^{\prime \prime}\right), \mathcal{B} \times \mathcal{A}_{3, m}}^{\prime}\left(q_{\varpi}\right)(w)=\sum_{\left(\varpi, \varpi^{\prime \prime}\right) \in \mathcal{B} \times \mathcal{A}_{3, m}} r^{\prime}(\varpi) r_{\varpi}^{\prime \prime}\left(\varpi^{\prime \prime}\right) \bar{I}\left(q_{\varpi}\right)\left(w \circ \psi_{\varpi} \circ \psi_{\varpi}\right) \\
& =\sum_{\varpi \in \mathcal{B}} r_{\varpi}^{\prime} \sum_{\varpi^{\prime \prime} \in \mathcal{A}_{3, m}} r_{\varpi}^{\prime \prime}\left(\varpi^{\prime \prime}\right) \bar{I}\left(q_{\varpi}\right)\left(w \circ \psi_{\varpi} \circ \psi_{\varpi \prime}\right)=\sum_{\varpi \in \mathcal{B}} r_{\varpi}^{\prime} S_{r_{\varpi}^{\prime \prime}}^{\prime}\left(q_{\varpi}\right)\left(w \circ \psi_{\varpi}\right) \\
& \geq \sum_{\varpi \in \mathcal{B}} r_{\varpi}^{\prime} \bar{I}\left(\widehat{\Lambda}_{r_{\varpi}^{\prime \prime}}\left(q_{\varpi}\right)\right)\left(\left.w\right|_{V(\mathcal{B})} \circ \psi_{\varpi}\right)=S_{r^{\prime}, \varpi, \mathcal{B}}^{\prime}\left(\widehat{\Lambda}_{r_{\varpi}^{\prime \prime}}\left(q_{\varpi}\right)\right)\left(\left.w\right|_{V(\mathcal{B})}\right)
\end{aligned}
$$

Now, if $w \in \mathcal{L}_{V\left(\mathcal{B} \times \mathcal{A}_{3, m}\right), M_{1}, M_{2}}$, then $\left.w\right|_{V(\mathcal{B})} \in \mathcal{L}_{V(\mathcal{B}), M_{1}, M_{2}}$. Therefore, by the definition of $(1 / \widehat{R})$, we have

$$
(1 / \widehat{R})\left(S_{r^{\prime}, \varpi, \mathcal{B}}\left(\widehat{\Lambda}_{r_{\varpi}^{\prime \prime}}\left(q_{\varpi}\right)\right)\right)_{\left\{M_{1}, M_{2}\right\}} \leq(1 / \widehat{R})\left(S_{r,(\varpi, \varpi \prime \prime), \mathcal{B} \times \mathcal{A}_{3, m}}\left(q_{\varpi}\right)\right)_{\left\{M_{1}, M_{2}\right\}} .
$$

Let now $v \in \mathcal{L}_{V(\mathcal{B}) ; M_{1}, M_{2}}$. For every $\varpi \in \mathcal{B}$ let $\tilde{v}_{\varpi} \in \mathcal{L}_{V\left(\mathcal{A}_{3, m}\right), V^{(0)}}\left(v \circ \psi_{\varpi}\right)$ be so that

$$
\bar{I}\left(\widehat{\Lambda}_{r_{\varpi}^{\prime \prime}}\left(q_{\varpi}\right)\right)\left(v \circ \psi_{\varpi}\right)=S_{r_{\varpi}^{\prime \prime}}^{\prime}\left(q_{\varpi}\right)\left(\tilde{v}_{\varpi}\right)
$$

and let $\bar{v} \in \mathbb{R}^{V\left(\mathcal{B} \times \mathcal{A}_{3, m}\right)}$ be defined by

$$
\bar{v}\left(\psi_{\varpi} \circ \psi_{\varpi^{\prime \prime}}(P)\right)=\tilde{v}_{\varpi}\left(\psi_{\varpi^{\prime \prime}}(P)\right)
$$

for every $\varpi \in \mathcal{B}, \varpi^{\prime \prime} \in \mathcal{A}_{3, m}, P \in V^{(0)}$. The definition is correct. In fact, if $\psi_{\varpi} \circ \psi_{\varpi^{\prime \prime}}(P)=$ $\psi_{\varpi_{1}} \circ \psi_{\varpi_{1}^{\prime \prime}}\left(P_{1}\right)$, then either $\varpi=\varpi_{1}$, thus $\psi_{\varpi^{\prime \prime}}(P)=\psi_{\varpi_{1}^{\prime \prime}}\left(P_{1}\right)$, or $\varpi \neq \varpi_{1}$, thus by Lemma $5.1, \psi_{\varpi^{\prime \prime}}(P), \psi_{\varpi_{1}^{\prime \prime}}\left(P_{1}\right) \in V^{(0)}$ and $\tilde{v}_{\varpi}\left(\psi_{\varpi^{\prime \prime}}(P)\right)=v\left(\psi_{\varpi} \circ \psi_{\varpi^{\prime \prime}}(P)\right)=v\left(\psi_{\varpi_{1}} \circ \psi_{\varpi_{1}^{\prime \prime}}\left(P_{1}\right)\right)=$ $\tilde{v}_{\varpi_{1}}\left(\psi_{\varpi_{1}^{\prime \prime}}\left(P_{1}\right)\right)$. It follows

$$
\bar{v} \circ \psi_{\varpi}=\tilde{v}_{\varpi}
$$

Moreover, we have $\bar{v} \in \mathcal{L}_{V\left(\mathcal{B} \times \mathcal{A}_{3, m}\right), M_{1}, M_{2}}$ and $\bar{v}=v$ on $V(\mathcal{B})$. In fact, if $Q \in V(\mathcal{B})$, then $Q=\psi_{\varpi}(P)$ for some $\varpi \in \mathcal{B}$ and $P \in V^{(0)}$. There exists $\varpi^{\prime \prime} \in \mathcal{A}_{3, m}$ such that $P=\psi_{\varpi^{\prime \prime}}(P)$. Thus,

$$
\bar{v}(Q)=\bar{v}\left(\psi_{\varpi}(P)\right)=\bar{v}\left(\psi_{\varpi} \circ \psi_{\varpi^{\prime \prime}}(P)\right)=\tilde{v}_{\varpi}\left(\psi_{\varpi^{\prime \prime}}(P)\right)=v \circ \psi_{\varpi}(P)=v(Q)
$$

Thus, by (7.6), (7.8) and (7.9) we have 


$$
\begin{aligned}
& S_{r,\left(\varpi, \varpi^{\prime \prime}\right), \mathcal{B} \times \mathcal{A}_{3, m}}^{\prime}\left(q_{\varpi}\right)(\bar{v})=\sum_{\varpi \in \mathcal{B}} r_{\varpi}^{\prime} S_{r_{\varpi}^{\prime \prime}}^{\prime \prime}\left(q_{\varpi}\right)\left(\bar{v} \circ \psi_{\varpi}\right) \\
= & \sum_{\varpi \in \mathcal{B}} r_{\varpi}^{\prime} \bar{I}\left(\widehat{\Lambda}_{r_{\varpi}^{\prime \prime}}\left(q_{\varpi}\right)\right)\left(v \circ \psi_{\varpi}\right)=S_{r^{\prime}, \varpi, \mathcal{B}}^{\prime}\left(\widehat{\Lambda}_{r_{\varpi}^{\prime \prime}}\left(q_{\varpi}\right)\right)(v)
\end{aligned}
$$

and, in view also of the definition $(1 / \widehat{R})$ and $(7.7)$, the Lemma is proved.

Corollary 7.11. Let $\mathcal{B}$ be a subset of $\mathcal{A}_{3, n}$. Let $q \in \widetilde{\mathcal{Q}}$. Let $\left.r^{\prime} \in\right] 0,+\infty\left[{ }^{\mathcal{B}}, r^{\prime \prime} \in\right] 0,+\infty\left[\mathcal{A}_{3, m}\right.$. Let $r \in] 0,+\infty\left[{ }^{\mathcal{B} \times \mathcal{A}_{3, m}}\right.$ be defined by $r\left(\varpi^{\prime}, \varpi^{\prime \prime}\right)=r^{\prime}\left(\varpi^{\prime}\right) r^{\prime \prime}\left(\varpi^{\prime \prime}\right)$. Let $M_{1}, M_{2}$ be nonempty disjoint subsets of $V(\mathcal{B})$. Then

$$
\widehat{R}\left(S_{r^{\prime}, \mathcal{B}}\left(\widehat{\Lambda}_{r^{\prime \prime}}(q)\right)\right)_{\left\{M_{1}, M_{2}\right\}}=\widehat{R}\left(S_{r, \mathcal{B} \times \mathcal{A}_{3, m}}(q)\right)_{\left\{M_{1}, M_{2}\right\}}
$$

\section{Eigenforms on General Fractals}

In this Section, we will prove the main Theorem. Following the ideas described at beginning of Section 6, we will evaluate the resistances along bigger and bigger sets. We will use the $n$-blocks $G_{l, n}^{(4)}$ with $n$ large enough for the reason explained there. We start by evaluating the resistance between $P_{j}$ and the relative bridge. We have to require that it is less then $\frac{a_{l}}{2}$ where the correct value of $a_{l}$ amounts to $\frac{2}{\#\left(\widetilde{B}_{l}^{\prime}\right)}$ (cf. Lemma 7.6). To this aim, we need to introduce a special constant $\bar{t}$ as follows

$$
\bar{t}=3 \max \left\{\widehat{R}(q)_{\left\{\left\{P_{j}\right\}, B\right\}}, q \in \widetilde{K}, j \in \mathcal{A}_{1}, \varnothing \neq B \subseteq V^{(0)}, P_{j} \notin B\right\} .
$$

Moreover, we will define a class of sets of weights in $W_{n}$ that, restricted to $\mathcal{A}_{2, n}$, only depend on the $\Pi_{n, n_{2}}$-image of the point. Namely, given

$$
\left.n>n_{2}, \quad g: \mathcal{A}_{3, n_{2}} \rightarrow\right] 0,+\infty\left[, \quad 0<\eta \leq \min \left\{\frac{1}{\#\left(\widetilde{B}_{l}^{\prime}\right)}\right\} \leq \frac{1}{2},\right.
$$

we define $r_{(g, \eta, n)} \in W_{n}$ by

$$
r_{(g, \eta, n)}(\varpi)=\left\{\begin{array}{ll}
\bar{t} & \text { if } \varpi=\left(j_{(n)}\right) \\
g\left(\Pi_{n, n_{2}}(\varpi)\right) & \text { otherwise }
\end{array} \quad \forall \varpi \in \mathcal{A}_{3, n} .\right.
$$

In the sequel, untill Lemma 8.11, we will consider $\eta$ as a fixed value (satisfying the required inequalities), and in the constants introduced $\left(\tilde{t}_{1, j, l, n}, t_{2, q, j, l, n}\right.$ and so on) we will not stress the dependance on $\eta$. Only in Lemma 8.12 we will choose a suitable, sufficiently small, value of $\eta$. 
Lemma 8.1. Given $n>n_{2}$ and $j \in \widetilde{B}_{l}^{\prime}$, there exists $\tilde{t}_{1, j, l, n} \geq \frac{\bar{t}}{\eta}$ such that, if $g=\tilde{t} \geq$ $\tilde{t}_{1, j, l, n}$ on $\bar{C}_{j}^{(1)}$ then $\widehat{R}\left(S_{r_{(g, \eta, n)}, \bar{G}_{j, n}^{(1)}}(q)\right)_{\left\{\left\{P_{j}\right\}, M_{j, l}\right\}} \leq \frac{\eta}{2} \leq \frac{1}{2 \#\left(\widetilde{B}_{l}^{\prime}\right)}$ for every $q \in \widetilde{K}$.

Proof. Let $\bar{\varpi} \in \bar{G}_{j, n}^{(1)}$ be such that there exists $\bar{Q} \in V_{\bar{\varpi}} \cap M_{j, l} \neq \varnothing$. As $\bar{G}_{j, n}^{(1)}$ is connected, there exists a simple path $\left(\varpi_{0}, \ldots, \varpi_{m}\right)$ in $\bar{G}_{j, n}^{(1)}$ connecting $\bar{\varpi}$ to $j_{(n)}$. Since $Q \in V\left(\bar{G}_{j, l, n}^{(21)}\right)$, then $Q \notin V_{\left(j_{n}\right)}$ by Lemma 5.5 , thus $\bar{\varpi} \neq j_{(n)}$, and $m \geq 1$. For every $v \in \mathbb{R}^{V\left(\bar{G}_{j, n}^{(1)}\right)}$, we have

$$
\begin{aligned}
S_{r_{(g, \eta, n)}, \bar{G}_{j, n}^{(1)}}^{\prime}(q)(v) & =\sum_{\varpi \in \bar{G}_{j, n}^{(1)}} r_{(g, \eta, n)}(\varpi) \bar{I}(q)\left(v \circ \psi_{\varpi}\right) \geq \sum_{s=0}^{m} r_{(g, \eta, n)}\left(\varpi_{s}\right) \bar{I}(q)\left(v \circ \psi_{\varpi_{s}}\right) \\
= & \left(g\left(j_{\left(n_{2}\right)}\right) \sum_{s=0}^{m-1} \bar{I}(q)\left(v \circ \psi_{\varpi_{s}}\right)\right)+\frac{\bar{t}}{\eta} \bar{I}(q)\left(v \circ \psi_{j_{(n)}}\right) \\
& \geq \tilde{t}_{1} \sum_{s=0}^{m-1} \bar{I}(q)\left(v \circ \psi_{\varpi_{s}}\right)+\frac{\bar{t}}{\eta} \bar{I}(q)\left(v \circ \psi_{j_{(n)}}\right)
\end{aligned}
$$

provided $g\left(j_{\left(n_{2}\right)}\right) \geq \tilde{t}_{1, j, l, n}$. Now, if $q \in \widetilde{K}$, we have $q_{\varpi} \geq \hat{q}_{\varpi}$ for every $\varpi \in J$, where $\hat{q} \in \widetilde{K} \subseteq \widetilde{\mathcal{Q}}$ is defined by

$$
\hat{q}_{\varpi}=1-\delta \text { if } \varpi \in \bar{J}, \hat{q}_{\varpi}=0 \text { if } \varpi \in J \backslash \bar{J} .
$$

Hence, $q_{\left\{j_{1}, j_{2}\right\}} \geq \hat{q}_{\left\{j_{1}, j_{2}\right\}}$ for every $\left\{j_{1}, j_{2}\right\} \in J$, thus $\bar{I}(q)(u) \geq \bar{I}(\hat{q})(u)$, hence,

$$
\begin{aligned}
& S_{r_{(g, \eta, n)}, \bar{G}_{j, n}^{(1)}}^{\prime}(q)(v) \geq \tilde{t}_{1} \sum_{s=0}^{m-1} \bar{I}(\hat{q})\left(v \circ \psi_{\varpi_{s}}\right)+\frac{\bar{t}}{\eta} \bar{I}(\hat{q})\left(v \circ \psi_{j_{(n)}}\right) \\
= & \tilde{t}_{1, j, l, n} S_{1, \bar{G}}^{\prime}(\hat{q})(v)+\frac{\bar{t}}{\eta} S_{1,\left\{j_{(n)}\right\}}^{\prime}(\hat{q})(v), \quad \bar{G}:=\left\{\varpi_{0}, \ldots, \varpi_{m-1}\right\}
\end{aligned}
$$

Let $M:=V_{\varpi_{m-1}} \cap V_{\varpi_{m}}=V_{\varpi_{m-1}} \cap V_{j_{(n)}} \neq \varnothing$. We have $P_{j} \notin M$, and let

$$
M^{\prime}:=M \cup\left\{P_{j}\right\} \subseteq V_{j_{(n)}}
$$

Suppose now $v \in \mathcal{L}_{V\left(\bar{G}_{j, n}^{(1)}\right) ;\left\{P_{j}\right\}, M_{j, l}}^{\prime}$. By the uniform continuity of $\geq\left. S_{1,\left\{j_{(n)}\right\}}^{\prime}(\hat{q})\right|_{M^{\prime}}$ we have $\left.S_{1,\left\{j_{(n)}\right\}}^{\prime}(\hat{q})\right|_{M^{\prime}}\left(\left.v\right|_{M^{\prime}}\right)>\left.\frac{2}{3} S_{1,\left\{j_{(n)}\right\}}^{\prime}(\hat{q})\right|_{M^{\prime}}\left(\left.\chi\right|_{M}\right)=\frac{2}{3}(1 / \widehat{R})\left(S_{1,\left\{j_{(n)}\right\}}(\hat{q})\right)_{\left\{P_{j}\right\}, M}$ provided $|v(Q)-1|<\eta$ on $M$ where $\eta$ is a suitable positive number. Thus,

$$
S_{1,\left\{j_{(n)}\right\}}^{\prime}(\hat{q})(v) \geq\left. S_{1,\left\{j_{(n)}\right\}}^{\prime}(\hat{q})\right|_{M^{\prime}}\left(\left.v\right|_{M^{\prime}}\right)>\frac{2}{3}(1 / \widehat{R})\left(S_{1,\left\{j_{(n)}\right\}}(\hat{q})\right)_{\left\{P_{j}\right\}, M} .
$$

As $M \subseteq \psi_{j_{(n)}}\left(V^{(0)}\right)$ we have $M=\psi_{j_{(n)}}(B)$ with $\varnothing \neq B \subseteq V^{(0)}$. Also, $P_{j} \notin B$, so that $\left\{P_{j}\right\}$ and $B$ are nptdss of $V^{(0)}$. Therefore, by Corollary 3.5, we have 


$$
\widehat{R}\left(S_{1,\{j(n)\}}(\hat{q})\right)_{\left\{P_{j}\right\}, M}=\widehat{R}(\hat{q})_{\left\{\left\{P_{j}\right\}, B\right\}} \leq \frac{\bar{t}}{3} .
$$

Summarizing, if $|v(Q)-1|<\eta$ for every $Q \in M$, then

$$
S_{r_{(g, \eta, n)}, \bar{G}_{j, n}^{(1)}}^{\prime}(q)(v) \geq \frac{\bar{t}}{\eta} S_{1,\left\{j_{(n)}\right\}}^{\prime}(\hat{q})(v)>\frac{\bar{t}}{\eta} \frac{2}{3}(1 / \widehat{R})\left(S_{1,\left\{j_{(n)}\right\}}(\hat{q})\right)_{\left\{P_{j}\right\}, M} \geq \frac{2}{\eta} .
$$

If, on the contrary, there exists $Q \in M$ such that $|v(Q)-1| \geq \eta$, then, $v \in T_{1}$, where

$T_{1}:=\left\{w \in \mathbb{R}^{V(\bar{G})}: w(Q)=1 \forall Q \in M_{j, l} \cap V(\bar{G}), \quad \exists Q \in M:|w(Q)-1| \geq \eta, 0 \leq w \leq 1\right\}$.

By a compactness argument, there exists $\min _{w \in T_{1}} S_{1, \bar{G}}^{\prime}(\hat{q})(w):=c_{1}>0$. Hence, $S_{1, \bar{G}}^{\prime}(\hat{q})(v) \geq$ $c_{1}$. As a consequence,

$$
S_{r_{(g, \eta, n)}, \bar{G}_{j, n}^{(1)}}^{\prime}(q)(v) \geq \tilde{t}_{1, j, l, n} S_{1, \bar{G}}^{\prime}(\hat{q})(v) \geq \tilde{t}_{1, j, l, n} c_{1}>\frac{2}{\eta}
$$

for sufficiently large $\tilde{t}_{1, j, l, n}$. Taking also into account (8.1), in any case, for such $\tilde{t}_{1, j, l, n}$ we have $\widehat{R}\left(S_{r_{(g, \eta, n)}, \bar{G}_{j, n}^{(1)}}(q)\right)_{\left\{\left\{P_{j}\right\}, M_{j, l}\right\}} \leq \frac{\eta}{2}$.

Next, following the scheme in Section 6, we will prove in Lemma 8.3 the equivalent of formula $R_{1}+R_{2}=\frac{a_{l}}{2}$ there. We need the following preliminary Lemma.

Lemma 8.2. For $j \in \widetilde{B}_{l}^{\prime}$,

$$
\widehat{R}\left(S_{1, \bar{G}_{j, l, n}^{(22)}}^{(q)}\right)_{\left\{M_{j, l}^{\prime}, M_{j, l}^{\prime \prime}\right\}} \underset{n \rightarrow+\infty}{\longrightarrow}+\infty .
$$

uniformly for $q \in \widetilde{K}$.

Proof. We use Corollary 7.11 with $n_{2}$ in place of $n, \bar{C}_{j, l}^{(22)}$ in place of $\mathcal{B}, n-n_{2}$ in place of $m, r^{\prime}=r^{\prime \prime}=1$ so that $r(\varpi)=1$ for every $\varpi \in \bar{G}_{j, l, n}^{(22)}$. We have

$$
\begin{gathered}
(1 / \widehat{R})\left(S_{1, \bar{G}_{j, l, n}^{(22)}}(q)\right)_{\left\{M_{j, l}^{\prime}, M_{j, l}^{\prime \prime}\right\}}=(1 / \widehat{R})\left(S_{1, \bar{C}_{j, l}^{(222)}}\left(\widehat{\Lambda}_{1 ; n-n_{2}}(q)\right)\right)_{\left\{M_{j, l}^{\prime}, M_{j, l}^{\prime \prime}\right\}} \\
\leq\left|\widehat{\Lambda}_{1 ; n-n_{2}}(q)\right|(1 / \widehat{R})\left(S_{1, \bar{C}_{j, l}^{(22)}}\left(1_{J}\right)\right)_{\left\{M_{j, l}^{\prime}, M_{j, l}^{\prime \prime}\right\}} \underset{n \rightarrow+\infty}{\longrightarrow} 0
\end{gathered}
$$

uniformly on $\widetilde{K}$, by (2.2) and Corollary 7.9, and the Lemma follows.

Lemma 8.3. For every $\eta$ as above, there exists $n_{1, \eta}>n_{2}$ such that, if $n \geq n_{1, \eta}$ and $j \in \widetilde{B}_{l}^{\prime}$, for every $q \in \widetilde{K}$ there exists a unique $t\left(=t_{2, q, j, l, n}\right) \geq \frac{\bar{t}}{\eta}$, continuously depending on $q$, such that if $g=\tilde{t} \geq \tilde{t}_{1, j, l, n}$ on $\bar{C}_{j}^{(1)}$ and $g=t$ on $\bar{C}_{j, l}^{(22)}$, we have 


$$
\widehat{R}\left(S_{r_{(g, \eta, n)}, \bar{G}_{j, l, n}^{(17)}}(q)\right)_{\left\{\left\{P_{j}\right\}, M_{j, l}\right\}}+\widehat{R}\left(S_{r_{(g, \eta, n)}, \bar{G}_{j, l, n}^{(22)}}(q)\right)_{\left\{M_{j, l}^{\prime}, M_{j, l}^{\prime \prime}\right\}}=\frac{1}{\#\left(\widetilde{B}_{l}^{\prime}\right)}
$$

Proof. By Lemma 8.1, we have

$$
\widehat{R}\left(S_{r_{(g, \eta, n)}, \bar{G}_{j, l, n}^{(17)}}(q)\right)_{\left\{\left\{P_{j}\right\}, M_{j, l}\right\}} \leq \widehat{R}\left(S_{r_{(g, \eta, n)}, \bar{G}_{j, n}^{(1)}}(q)\right)_{\left\{\left\{P_{j}\right\}, M_{j, l}\right\}}<\frac{1}{\#\left(\widetilde{B}_{l}^{\prime}\right)}
$$

for every $q \in \widetilde{K}$. On the other hand,

$$
\widehat{R}\left(S_{r_{(g, \eta, n)}, \bar{G}_{j, l, n}^{(22)}}(q)\right)_{\left\{M_{j, l}^{\prime}, M_{j, l}^{\prime \prime}\right\}}=\widehat{R}\left(S_{t, \bar{G}_{j, l, n}^{(22)}}(q)\right)_{\left\{M_{j, l}^{\prime}, M_{j, l}^{\prime \prime}\right\}}=\frac{1}{t} \widehat{R}\left(S_{1, \bar{G}_{j, l, n}^{(22)}}(q)\right)_{\left\{M_{j, l}^{\prime}, M_{j, l}^{\prime \prime}\right\}}
$$

and, by Lemma 8.2, for sufficiently large $n, \widehat{R}\left(S_{1, \bar{G}_{j, l, n}^{(22)}}(q)\right)_{\left\{M_{j, l}^{\prime}, M_{j, l}^{\prime \prime}\right\}}>\frac{\bar{t}}{\eta} \frac{1}{\#\left(\widetilde{B}_{l}^{\prime}\right)}$ for every $q \in \widetilde{K}$. As by $(6.1)$ we have $\#\left(\widetilde{B}_{l}^{\prime}\right) \geq 2$, and $\widehat{R}\left(S_{1, \bar{G}_{j, l, n}^{(22)}}(q)\right)_{\left\{M_{j, l}^{\prime}, M_{j, l}^{\prime \prime}\right\}}$ is finite by Lemma 3.2 , we conclude with

$$
t=\frac{\widehat{R}\left(S_{1, \bar{G}_{j, l, n}^{(22)}}(q)\right)_{\left\{M_{j, l}^{\prime}, M_{j, l}^{\prime \prime}\right\}}}{\frac{1}{\#\left(\widetilde{B}_{l}^{\prime}\right)}-\widehat{R}\left(S_{r_{(g, \eta, n)}, \bar{G}_{j, l, n}^{(17)}}(q)\right)_{\left\{\left\{P_{j}\right\}, M_{j, l}\right\}}} \cdot \mathbf{.}
$$

We will now prove that the resistance between two points of $V^{(0)}$ approximates $\widetilde{\bar{R}}$. In the following Lemma we will see this when the points are in the same block. We will get this using two times Lemma 3.7 in the situation of Lemma 8.3.

Lemma 8.4. For every $\left.\eta \in] 0, \min \left\{\frac{1}{\#\left(\widetilde{B}_{l}^{\prime}\right)}\right\}\right]$ and $n \geq n_{1, \eta}$, there exist $\tilde{t}_{2, n}, \tilde{t}_{3, n}>\frac{\bar{t}}{\eta}$ such that if $q \in \widetilde{K}, g=\tilde{t}_{1, n}:=\max \left\{\tilde{t}_{1, j, l, n}: j, l\right\}$ on $\bar{C}_{j}^{(1)}, g=\tilde{t}_{2, n}$ on $\bar{C}_{j, l}^{(21)}, g=\tilde{t}_{3, n}$ on $\bar{C}_{l}^{(3)}$, $g=t_{2, q, j, l, n}$ on $\bar{C}_{j, l}^{(22)}$ for every $l=1, \ldots, h^{\prime}, j \in \widetilde{B}_{l}^{\prime}$, then for every $l=1, \ldots, h^{\prime}, j_{1}, j_{2} \in \widetilde{B}_{l}^{\prime}$, $j_{1} \neq j_{2}$

i) $\frac{2}{\#\left(\widetilde{B}_{l}^{\prime}\right)}-3 \eta<\widehat{R}\left(S_{r_{(g, \eta, n)}, \bar{G}_{n}^{(5)}}(q)\right)_{\left\{P_{j_{1}}, P_{j_{2}}\right\}}<\frac{2}{\#\left(\widetilde{B}_{l}^{\prime}\right)}+3 \eta$.

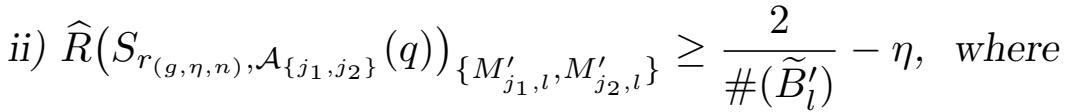

$$
\mathcal{A}_{\left\{j_{1}, j_{2}\right\}}:=\bar{G}_{n}^{(5)} \backslash\left(\bar{G}_{j_{1}, l, n}^{(1217)} \cup \bar{G}_{j_{2}, l, n}^{(1217)}\right) .
$$

Proof. For $j \in \widetilde{B}_{l}^{\prime}$, we use Lemma 3.7 with

$$
\mathcal{B}_{1}:=\bar{G}_{j, l, n}^{(17)}, \quad \mathcal{B}_{2}:=\bar{G}_{j, l, n}^{(22)}, \quad \mathcal{B}_{3}:=\bar{G}_{j, l, n}^{(21)}, \quad \mathcal{B}_{4}:=\varnothing
$$




$$
A_{1}=\left\{P_{j}\right\}, A_{2}=M_{j, l}^{\prime \prime}
$$

and obtain that

$$
\begin{gathered}
M_{1}=V\left(\bar{G}_{j, l, n}^{(17)}\right) \cap V\left(\bar{G}_{j, l, n}^{(21)}\right)=V\left(\bar{G}_{j, n}^{(1)}\right) \cap V\left(\bar{G}_{j, l, n}^{(21)}\right)=M_{j, l}, \\
M_{2}=V\left(\bar{G}_{j, l, n}^{(21)}\right) \cap V\left(\bar{G}_{j, l, n}^{(22)}\right)=M_{j, l}^{\prime},
\end{gathered}
$$

Note that the second equality in (8.2) is a simple consequence of Lemma 6.8, case e). Next, by Lemma 6.8 again, $\mathcal{B}_{1}$ and $\mathcal{B}_{2}$ are separated so that we can in fact apply Lemma 3.7 , and moreover, $\mathcal{B}_{5} \subseteq \bar{G}_{j, n}^{(1)} \cup \bar{G}_{j, l, n}^{(22)}$. We note that, if $\varpi \in \mathcal{B}_{5}$, then, by definition, we have $r_{(g, \eta, n)}(\varpi)=\tilde{t}$ or $r_{(g, \eta, n)}=\frac{\bar{t}}{\eta}$ or $r_{(g, \eta, n)}(\varpi)=t_{2, q, j, l, n}$. Once we fix $n$ and $\eta$, such numbers are bounded independently of $q \in \widetilde{K}$. In fact, $t_{2, q, j, l, n}$ dependes continuously on $q$, thus it has a maximum on $\widetilde{K}$. Now, for every $j \in \widetilde{B}_{l}^{\prime}$, put

$$
G_{j}=: \bar{G}_{j, l, n}^{(17)} \cup \bar{G}_{j, l, n}^{(21)} \cup \bar{G}_{j, l, n}^{(22)}=\bar{G}_{j, n}^{(1)} \cup \bar{G}_{j, l, n}^{(7)} \cup \bar{G}_{j, l, n}^{(21)} \cup \bar{G}_{j, l, n}^{(22)}
$$

We now use Lemma 3.7 ii) and, for sufficiently large $\tilde{t}_{2, n}$, and for every $q \in \widetilde{K}$, we get

$$
\frac{1}{\#\left(\widetilde{B}_{l}^{\prime}\right)}-\eta<\widehat{R}\left(S_{r_{(g, \eta, n)}, G_{j}}(q)\right)_{\left\{\left\{P_{j}\right\}, M_{j, l}^{\prime \prime}\right\}}<\frac{1}{\#\left(\widetilde{B}_{l}^{\prime}\right)}+\eta
$$

Note that the sets $G_{j}$ are mutually disjoint by Lemma 6.6 ii). Thus, we use Lemma 3.7 again with

$$
\mathcal{B}_{1}:=G_{j_{1}}, \quad \mathcal{B}_{2}:=G_{j_{2}}, \quad \mathcal{B}_{3}:=\bar{G}_{l, n}^{(3)}, \quad \mathcal{B}_{4}:=\bigcup_{j \in \widetilde{B}_{l}^{\prime} \backslash\left\{j_{1}, j_{2}\right\}} G_{j}
$$

$A_{1}=\left\{P_{j_{1}}\right\}, A_{2}=\left\{P_{j_{2}}\right\}$. We easily see that $\mathcal{B}_{1}, \mathcal{B}_{2}, \mathcal{B}_{3}, \mathcal{B}_{4}$ are mutually disjoint subsets of $\mathcal{A}_{3, n}$. Moreover, $\mathcal{B}_{1}, \mathcal{B}_{2}$ and $\mathcal{B}_{4}$ are mutually separated by Lemma 6.8 . Finally, by Lemma 6.8 again, $M_{1}:=V\left(\mathcal{B}_{1}\right) \cap V\left(\mathcal{B}_{3}\right)=M_{j_{1}, l}^{\prime \prime}$, and similarly, $M_{2}=M_{j_{2}, l}^{\prime \prime}$. In conclusion, all hypotheses of Lemma $3.7 \mathrm{ii})$ are satisfied. Note also, that $\mathcal{B}_{5} \subseteq \bigcup_{j \in \widetilde{B}_{l}^{\prime}} \bar{G}_{j, l, n}^{(22)}$ by Lemma 6.8 , and $\mathcal{A}=\bar{G}_{n}^{(5)}$. We have $r_{(g, \eta, n)}(\varpi)=t_{2, q, j, l, n} \leq A$ for every $\varpi \in \mathcal{B}_{5}$, where $A$ is a suitable constant. By Lemma 3.7, also using (8.3) both with $j=j_{1}$ and with $j=j_{2}$, we have

$$
\begin{gathered}
\frac{2}{\#\left(\widetilde{B}_{l}^{\prime}\right)}-3 \eta< \\
\widehat{R}\left(S_{r_{(g, \eta, n)}, G_{j_{1}}}(q)\right)_{\left\{\left\{P_{\left.\left.j_{1}\right\}, M_{j_{1}, l}^{\prime \prime}\right\}}+\widehat{R}\left(S_{r_{(g, \eta, n)}, G_{j_{2}}}(q)\right)_{\left\{\left\{P_{j_{2}}\right\}, M_{j_{2}, l}^{\prime \prime}\right\}}-\eta \leq\right.\right.}{\widehat{R}\left(S_{r_{(g, \eta, n)}, \bar{G}_{n}^{(5)}}(q)\right)_{\left\{\left\{P_{j_{1}}\right\},\left\{P_{j_{2}}\right\}\right\}} \leq} \leq \\
\widehat{R}\left(S_{r_{(g, \eta, n)}, G_{j_{1}}}(q)\right)_{\left\{\left\{P_{\left.\left.j_{1}\right\}, M_{j_{1}, l}^{\prime \prime}\right\}}+\widehat{R}\left(S_{r_{(g, \eta, n)}, G_{j_{2}}}(q)\right)_{\left\{\left\{P_{\left.\left.j_{2}\right\}, M_{j_{2}, l}^{\prime \prime}\right\}}\right.\right.}+\eta\right.\right.}
\end{gathered}
$$




$$
<\frac{2}{\#\left(\widetilde{B}_{l}^{\prime}\right)}+3 \eta
$$

for every $q \in \widetilde{K}$, for sufficiently large $\tilde{t}_{3, n}$ for every $l$ and $j_{1}, j_{2} \in \widetilde{B}_{l}^{\prime}$, and i) is proved.

We are now going to prove ii). In this case, we use Lemma $3.7 \mathrm{i}$ ) with

$$
\begin{gathered}
\mathcal{B}_{1}=\bar{G}_{j_{1}, l, n}^{(22)}, \quad \mathcal{B}_{2}=\bar{G}_{j_{2}, l, n}^{(22)}, \quad \mathcal{B}_{3}:=\bar{G}_{l, n}^{(3)}, \quad \mathcal{B}_{4}:=\bigcup_{j \in \widetilde{B_{l}^{\prime} \backslash\left\{j_{1}, j_{2}\right\}}} G_{j} \\
A_{1}=M_{j_{1}, l}^{\prime}, \quad A_{2}=M_{j_{2}, l}^{\prime}
\end{gathered}
$$

We easily see that the hypothesis of Lemma 3.7 is in fact satisfied. Moreover, $M_{1}=M_{j_{1}, l}^{\prime \prime}$, $M_{2}=M_{j_{2}, l}^{\prime \prime}$, and $\mathcal{A}=\mathcal{A}_{j_{1}, j_{2}}$. We conclude

$$
\begin{aligned}
& \widehat{R}\left(S_{r_{(g, \eta, n)}, \mathcal{A}_{j_{1}, j_{2}}}(q)\right)_{\left\{M_{j_{1}, l}^{\prime}, M_{j_{2}, l}^{\prime}\right\}} \geq \\
& \widehat{R}\left(S_{r_{(g, \eta, n)}, \bar{G}_{j_{1}, l, n}^{(22)}}(q)\right)_{\left\{M_{j_{1}, l}^{\prime}, M_{j_{1}, l}^{\prime \prime}\right\}}+\widehat{R}\left(S_{r_{(g, \eta, n)}, \bar{G}_{j_{2}, l, n}^{(22)}}(q)\right)_{\left\{M_{j_{2}, l}^{\prime}, M_{j_{2}, l}^{\prime \prime}\right\}} \\
& =\frac{2}{\#\left(\widetilde{B}_{l}^{\prime}\right)}-\left(\widehat{R}\left(S_{r_{(g, \eta, n)}, \bar{G}_{j_{1}, l, n}^{(17)}}(q)\right)_{\left\{\left\{P_{j_{1}}\right\}, M_{j_{1}, l}\right\}}+\widehat{R}\left(S_{r_{(g, \eta, n)}, \bar{G}_{j_{2}, l, n}^{(17)}}(q)\right)_{\left\{\left\{P_{j_{2}}\right\}, M_{j_{2}, l}\right\}}\right)
\end{aligned}
$$

by Lemma 8.3, and by Lemma 8.1 and Remark 3.6, we have

$$
\widehat{R}\left(S_{r_{(g, \eta, n)}, \bar{G}_{j, l, n}^{(17)}}(q)\right)_{\left\{\left\{P_{j}\right\}, M_{j, l}\right\}} \leq \widehat{R}\left(S_{r_{(g, \eta, n)}, \bar{G}_{j, n}^{(1)}}(q)\right)_{\left\{\left\{P_{j}\right\}, M_{j, l}\right\}} \leq \frac{\eta}{2}
$$

for $j=j_{1}, j_{2}$, and ii) follows.

We have now to prove that the effective resistance (denoted in this discussion by $R_{j_{1}, j_{2}}$ ) between two points $P_{j_{1}}, P_{j_{2}}$ of $V^{(0)}$ not in the same block approximates $\widetilde{\bar{R}}_{\left\{j_{1}, j_{2}\right\}}$. In order to do this, we need some preliminary considerations. If $j_{1}, j_{2}$ are not in a common block, which means that they do not belong to a common $\widetilde{B}_{l}^{\prime}$, by Lemma 7.5 we can assume $\left(j_{1}, j_{2}\right)$ is an $\bar{l}$-pair for some $\bar{l}$, so that we can suppose by a recursive argument we have information about $R_{j_{1}, \tilde{j}_{\bar{l}}}$ and $R_{j_{1}, \tilde{j}_{\bar{l}}}$, and we have to combine such information to investigate $R_{j_{1}, j_{2}}$. We will easily deduce, in view of Lemma 8.4 and of the triangular inequality of the effective resistance, that $R_{\left\{j_{1}, j_{2}\right\}}$ does not exceed $\widetilde{\bar{R}}_{j_{1}, j_{2}}+$ a small quantity, and we have to find a bound from below. The way to obtain the bound from below turns out to be rather long and technical. However, I do not see a simpler proof. A possible alternative approach would be to replace the set $\widetilde{K}$ by a set where the conductivities $q_{\left\{j_{1}, j_{2}\right\}}$ approximate those of $\bar{q}$, that is 1 , for vertices in the same block, and amount to 0 for vertices in different blocks, but it does not appear to be simple to prove that such a set is mapped into itself in the general case.

To find the bound from below, we first need to prove a separation property of $P_{\tilde{j}_{\bar{l}}}$. To describe it more precisely, observe that the set $\bar{G}_{\tilde{j}_{\bar{l}}, n}^{(121)}$ can be seen as the union of the vertex part $\bar{G}_{\tilde{j}_{\bar{l}}, n}^{(1)}$ and some branches, that is the exterior parts $\bar{G}_{\tilde{j}_{\bar{l}}, l, n}^{(21)}$ of the bridges. In Lemma 
8.5 we will prove that every point not in $\bar{G}_{\tilde{j}_{\bar{l}}, n}^{(121)}$ can be connected to precisely one branch, and in Lemma 8.7, in view of the final paragraph in Remark 8.6, we in particular prove that $P_{j_{1}}$ and $P_{j_{2}}$ are connected to different branches, so that they lie in different parts with respect to $P_{\tilde{j}_{\bar{l}}}$.

Lemma 8.5. For every $\varpi \in \bar{G}_{n}^{(5)} \backslash \bar{G}_{\tilde{j}_{\bar{l}}, n}^{(121)}$ there exists precisely one $l$ with $\tilde{j}_{\bar{l}} \in \widetilde{B}_{l}^{\prime}$ such that $\varpi$ is connected in $\bar{G}_{n}^{(5)} \backslash \bar{G}_{\tilde{j}_{\bar{l}}, n}^{(121)}$ to points in $\bar{G}_{\tilde{j}_{\bar{l}}, l, n}^{(121)}$.

Proof. There exists a simple path connecting $\varpi$ to $\left(\tilde{j}_{\bar{l}}\right)_{(n)} \in \bar{G}_{\tilde{j}_{\bar{l}}, n}^{(1)}$ in $\bar{G}_{n}^{(5)}$, and the first vertex in the path lying in $\bar{G}_{\tilde{j}_{\bar{l}}, n}^{(121)}$, in fact belongs to $\bar{G}_{\tilde{j}_{\bar{l}}, l, n}^{(121)}$ for some $l$ and the existence is proved. We now prove the uniqueness: Suppose by contradiction that there exist two different $l_{i}, i=1,2$ with $\tilde{j}_{\bar{l}} \in \widetilde{B}_{l_{i}}^{\prime}$ such that $\varpi$ is connected in $\bar{G}_{n}^{(5)} \backslash \bar{G}_{\tilde{j}_{\bar{l}}, n}^{(121)}$ to some point in $\bar{G}_{\tilde{j}_{\bar{l}}, l_{i}, n}^{(121)}$, and by Lemma 6.8 such a point belongs to $\bar{G}_{\tilde{j}_{\bar{l}}, l_{i}, n}^{(21)}$. Then there exists a path in $\bar{G}_{n}^{(5)} \backslash \bar{G}_{\tilde{j}_{\bar{l}}, n}^{(121)}$ connecting some point $\varpi_{-} \in \bar{G}_{\tilde{j}_{\bar{l}}, l_{1}, n}^{(21)}$ to some point $\varpi_{+} \in \bar{G}_{\tilde{j}_{\bar{l}}, l_{2}, n}^{(21)}$, passing through $\varpi$, and by Remark 6.7, $\varpi_{-} \notin \bar{G}_{\tilde{j}_{\bar{l}}, l_{2}, n}^{(7)}$. On the other hand, by Lemma $\left.6.5 \mathrm{i}\right)$, there exists a path connecting $\varpi_{-}$to some point of $\bar{G}_{\tilde{j}_{\bar{l}}, n}^{(1)}$ in $\bar{G}_{\tilde{j}_{\bar{l}}, l_{1}, n}^{(8)} \backslash \bar{G}_{\tilde{j}_{\bar{l}}, n}^{(1)} \subseteq \bar{G}_{n}^{(5)} \backslash \bar{G}_{l_{2}, n}^{(4)}$, and by Remark $6.7 \varpi_{-} \in \bar{G}_{\tilde{j}_{\bar{l}}, l_{2}, n}^{(7)}$, a contradiction, and the uniqueness is proved.

We say that two pairs $\left(j_{1}, l_{1}\right)$ and $\left(j_{2}, l_{2}\right)$ are $\left(\bar{l}_{1}, \bar{l}_{2}\right)$-related if $j_{1} \in \widetilde{B}_{l_{1}}^{\prime}, j_{2} \in \widetilde{B}_{l_{2}}^{\prime}, \bar{l}_{1} \leq \bar{l}_{2}$, $j_{1} \neq j_{2}, l_{1}, l_{2} \in\left[\bar{l}_{1}, \bar{l}_{2}\right]$, and for every $\varpi \in \bar{G}_{j_{1}, l_{1}, n}^{(22)}, \varpi^{\prime} \in \bar{G}_{j_{2}, l_{2}, n}^{(22)}$ there exists a path in $\left(\bigcup_{l=\bar{l}_{1}}^{\bar{l}_{2}} \bar{G}_{l, n}^{(4)}\right) \backslash\left(\bar{G}_{j_{1}, n}^{(121)} \cup \bar{G}_{j_{2}, n}^{(121)}\right)$ connecting $\varpi$ and $\varpi^{\prime}$. Note that in such a case, since $\varpi, \varpi^{\prime} \in\left(\bigcup_{l=\bar{l}_{1}}^{\bar{l}_{2}} \bar{G}_{l, n}^{(4)}\right) \backslash\left(\bar{G}_{j_{1}, n}^{(121)} \cup \bar{G}_{j_{2}, n}^{(121)}\right)$, the path is contained in $\left(\bigcup_{l=\bar{l}_{1}}^{\bar{l}_{2}} \bar{G}_{l, n}^{(4)}\right) \backslash\left(\bar{G}_{j_{1}, n}^{(121)} \cup \bar{G}_{j_{2}, n}^{(121)}\right)$, We will write shortly $\bar{l}_{2}$-related for $\left(1, \bar{l}_{2}\right)$-related.

Remark 8.6. Since $\bar{C}_{j, l}^{(21)} \cup \bar{C}_{j, l}^{(22)}$ is a path, thus is connected, the set $\bar{G}_{j, l, n}^{(21)} \cup \bar{G}_{j, l, n}^{(22)}$ is also connected. Therefore, there exist $\varpi \in \bar{G}_{j, l, n}^{(21)}, \varpi^{\prime} \in \bar{G}_{j, l, n}^{(22)}$ such that $\varpi \sim \varpi^{\prime}$. Thus, if $\left(j_{1}, l_{1}\right)$ and $\left(j_{2}, l_{2}\right)$ are $\left(\bar{l}_{1}, \bar{l}_{2}\right)$-related, then for every $\varpi \in \bar{G}_{j_{1}, l_{1}, n}^{(22)}$, there exists $\varpi^{\prime} \in \bar{G}_{j_{2}, l_{2}, n}^{(21)}$ and a path connecting $\varpi$ to $\varpi^{\prime}$ in $\left(\bigcup_{l=\bar{l}_{1}}^{\bar{l}_{2}} \bar{G}_{l, n}^{(4)}\right) \backslash\left(\bar{G}_{j_{1}, n}^{(121)} \cup \bar{G}_{j_{2}, n}^{(121)}\right)$.

By a similar argument, we see that, if $\left(j_{1}, l_{i}\right)$ and $\left(\tilde{j}_{\bar{l}} \cdot l_{2}\right)$ are $\left(\bar{l}_{1}, \bar{l}_{2}\right)$-related, then, in Lemma 8.5 for $\varpi=\left(j_{1}\right)_{n}$ we have $l=l_{2}$.

\section{Lemma 8.7}

i) If $l=1, \ldots, h^{\prime}$ and $j_{1}, j_{2} \in \widetilde{B}_{l}^{\prime}, j_{1} \neq j_{2}$, then $\left(j_{1}, l\right)$ and $\left(j_{2}, l\right)$ are $(l, l)$-related. 
ii) If $\left(j_{1}, j_{2}\right)$ is a $\bar{l}$-pair, and $\left(j_{1}, l_{1}\right)$ and $\left(\tilde{j}_{\bar{l}}, l_{2}\right)$ are $\bar{l}$-related, and $\left(\tilde{j}_{\bar{l}}, \bar{l}+1\right)$ and $\left(j_{2}, \bar{l}+1\right)$ are $(\bar{l}+1, \bar{l}+1)$-related, then $\left(j_{1}, l_{1}\right)$ and $\left(j_{2}, \bar{l}+1\right)$ are $(\bar{l}+1)$-related.

iii) For every $j_{1}, j_{2} \in \bigcup_{l=1}^{\bar{l}} \widetilde{B}_{l}^{\prime}, j_{1} \neq j_{2}$, then there exist $l_{1}, l_{2}=1, \ldots, \bar{l}$ such that $\left(j_{1}, l_{1}\right)$ and $\left(j_{2}, l_{2}\right)$ are $\bar{l}$-related.

iv) If $\left(j_{1}, j_{2}\right)$ is a $\bar{l}$-pair, then there exist $l_{1}, l_{2}$ such that $\left(j_{1}, l_{1}\right)$ and $\left(\tilde{j}_{\bar{l}}, l_{2}\right)$ are $\bar{l}$-related. Moreover, $\left(\tilde{j}_{\bar{l}}, \bar{l}+1\right)$ and $\left(j_{2}, \bar{l}+1\right)$ are $(\bar{l}+1, \bar{l}+1)$-related.

Proof. The set $\bar{C}_{j_{1}, l}^{(22)} \cup \bar{C}_{j_{2}, l}^{(22)} \cup \bar{C}_{l}^{(3)}$ is connected, as $\bar{C}_{j_{1}, l}^{(22)}$ and $\bar{C}_{j_{2}, l}^{(22)}$ are paths not separated from the connected set $\bar{C}_{l}^{(3)}$. As a consequence $\bar{G}_{j_{1}, l, n}^{(22)} \cup \bar{G}_{j_{2}, l, n}^{(22)} \cup \bar{G}_{l, n}^{(3)}$ is connected, thus we have proved i). We now prove ii). Given $\varpi \in \bar{G}_{j_{1}, l_{1}, n}^{(22)}$ and $\varpi^{\prime} \in \bar{G}_{j_{2}, \bar{l}+1, n}^{(22)}$, by hypothesis, there exists a path connecting $\varpi$ to any $\varpi^{\prime \prime} \in \bar{G}_{\tilde{j}_{\bar{l}}, l_{2}, n}^{(22)}$ contained in $\left(\bigcup_{l=1}^{\bar{l}} \bar{G}_{l, n}^{(4)}\right) \backslash\left(\bar{G}_{j_{1}, n}^{(121)} \cup\right.$ $\left.\bar{G}_{\tilde{j}_{\bar{l}}, n}^{(121)}\right)$, thus contained in $\left(\bigcup_{l=1}^{\bar{l}+1} \bar{G}_{l, n}^{(4)}\right) \backslash\left(\bar{G}_{j_{1}, n}^{(121)} \cup \bar{G}_{j_{2}, n}^{(121)}\right)$, as, by the definition of $\bar{l}$-pair, $j_{2} \notin \bigcup_{l=1}^{\bar{l}} \widetilde{B}_{l}^{\prime}$, thus the points of $\bar{G}_{j_{2}, n}^{(121)}$ do not belong to $\bigcup_{l=1}^{\bar{l}} \bar{G}_{l, n}^{(4)}$. Moreover, $l_{1}, l_{2} \leq \bar{l}$. Similarly, there exists a path connecting $\varpi^{\prime}$ to any $\varpi^{\prime \prime \prime} \in \bar{G}_{\tilde{j}_{\bar{l}}, \bar{l}+1, n}^{(22)}$ contained in $\bar{G}_{\bar{l}+1, n}^{(4)} \backslash$ $\left(\bar{G}_{j_{2}, n}^{(121)} \cup \bar{G}_{\tilde{j}_{\bar{l}}, n}^{(121)}\right)$, thus contained in $\left(\bigcup_{l=1}^{\bar{l}+1} \bar{G}_{l, n}^{(4)}\right) \backslash\left(\bar{G}_{j_{1}, n}^{(121)} \cup \bar{G}_{j_{2}, n}^{(121)}\right)$, as, by the definition of $\bar{l}$-pair, $j_{1} \notin \widetilde{B}_{\bar{l}+1}^{\prime}$, thus the points of $\bar{G}_{j_{1}, n}^{(121)}$ do not belong to $\bar{G}_{\bar{l}+1, n}^{(4)}$. Moreover, we can choose $\varpi^{\prime \prime} \sim \tilde{\varpi}^{\prime \prime} \in \bar{G}_{\tilde{j}_{\bar{l}}, l_{2}, n}^{(21)} \subseteq \bar{G}_{\tilde{j}_{\bar{l}}, l_{2}, n}^{(121)}$, and $\varpi^{\prime \prime \prime} \sim \tilde{\varpi}^{\prime \prime \prime} \in \bar{G}_{\tilde{j}_{\bar{l}}, \bar{l}+1, n}^{(21)} \subseteq \bar{G}_{\tilde{j}_{\bar{l}}, \bar{l}+1, n}^{(121)}$. By Lemma 6.11 , there exists a path contained in $\bar{G}_{\tilde{j}_{\bar{l}}, l_{2}, n}^{(121)} \cup \bar{G}_{\tilde{j}_{\bar{l}}, \bar{l}+1, n}^{(121)}$, thus contained in $\left(\bigcup_{l=1}^{\bar{l}+1} \bar{G}_{l, n}^{(4)}\right) \backslash$ $\left(\bar{G}_{j_{1}, n}^{(121)} \cup \bar{G}_{j_{2}, n}^{(121)}\right)$, connecting $\tilde{\varpi}^{\prime \prime}$ to $\tilde{\varpi}^{\prime \prime \prime}$. We thus obtain a path connecting $\varpi$ to $\varpi^{\prime}$ in $\left(\bigcup_{l=1}^{\bar{l}+1} \bar{G}_{l, n}^{(4)}\right) \backslash\left(\bar{G}_{j_{1}, n}^{(121)} \cup \bar{G}_{j_{2}, n}^{(121)}\right)$, and ii) is proved. We prove iii) by recursion on $\bar{l}$. If $\bar{l}=1$, then iii) follows from i). Suppose iii) holds for $l^{\prime}=1, \ldots, \bar{l}$, and let $j_{1}, j_{2} \in \bigcup_{l=1}^{\bar{l}+1} \widetilde{B}_{l}^{\prime}, j_{1} \neq j_{2}$. Then by Lemma 7.5, either $j_{1}, j_{2} \in \widetilde{B}_{l}^{\prime}$ for some $l=1, \ldots, \bar{l}+1$, and iii) follows from i) again, or $\left(j_{1}, j_{2}\right)$ or $\left(j_{2}, j_{1}\right)$ is an $l^{\prime}$-pair for some $l^{\prime}=1, \ldots, \bar{l}$. If for example, $\left(j_{1}, j_{2}\right)$ is an $l^{\prime}$-pair $j_{1}, \tilde{j}_{l^{\prime}} \in \bigcup_{l=1}^{l^{\prime}} \widetilde{B}_{l}^{\prime}$, and by the hypothesis there exist $l_{1}, l_{2}=1, \ldots, l^{\prime}$ such that $\left(j_{1}, l_{1}\right)$ and $\left(\tilde{j}_{l^{\prime}}, l_{2}\right)$ are $l^{\prime}$-related. Also, as $\tilde{j}_{l^{\prime}}, j_{2} \in \widetilde{B}_{l^{\prime}+1}^{\prime}$, by i) $\left(\tilde{j}_{l^{\prime}}, l^{\prime}+1\right)$ and $\left(j_{2}, l^{\prime}+1\right)$ are $\left(l^{\prime}+1, l^{\prime}+1\right)$-related. By ii) $\left(j_{1}, l_{1}\right)$ and $\left(j_{2}, l^{\prime}+1\right)$ are $(\bar{l}+1)$-related, and iii) is proved. As $j_{1}, \tilde{j}_{\bar{l}} \in \bigcup_{l=1}^{\bar{l}} \widetilde{B}_{l}^{\prime}$, and $j_{2}, \tilde{j}_{\bar{l}} \in \widetilde{B}_{\bar{l}+1}^{\prime}$, iv) is an immediate consequence of iii) and i) (it can be also deduced by the argument of the proof of iii)). 
Now, in order to obtain a good bound from below for $R_{\left\{j_{1}, j_{2}\right\}}$, we in fact will prove a sort of reverse-triangular inequality, but where the effective effective resistance between $P_{j_{1}}$ and $P_{j_{2}}$ is replaced by the effective resistance between $M_{j_{1}, l_{1}}^{\prime}$ and $M_{j_{2}, \bar{l}+1}^{\prime}$, that only slightly differs from it, and similarly for the other resistances in the formula (see (8.22) in Lemma 8.10 for the precise statement). We obtain such an inequality using Lemma 3.7 i). Lemmas 8.8 and 8.9 will assure that the hypothesis of Lemma 3.7 i) are satisfied in the present case.

Lemma 8.8. Suppose $\left(j_{1}, j_{2}\right)$ in an $\bar{l}$-pair, and let $l_{1}$ be as in Lemma $\left.8.7 \mathrm{iv}\right)$. Then the sets $\bar{G}_{j_{1}, l_{1}, n}^{(1217)}, \bar{G}_{j_{2}, \bar{l}+1, n}^{(1217)}$ and $\bar{G}_{\tilde{j}_{\bar{l}}, n}^{(121)}$ are mutually separated.

Proof. We have to prove that, if $\varpi \in \bar{G}_{j_{1}, l_{1}, n}^{(121)}, \varpi^{\prime} \in \bar{G}_{j_{2}, \bar{l}+1, n}^{(1217)}, \varpi^{\prime \prime} \in \bar{G}_{\tilde{j}_{\bar{l}}, n}^{(121)}$, then

$$
V_{\varpi} \cap V_{\varpi^{\prime}}=V_{\varpi} \cap V_{\varpi^{\prime \prime}}=V_{\varpi^{\prime}} \cap V_{\varpi^{\prime \prime}}=\varnothing .
$$

Recalling the definitions of $\bar{G}_{j_{1}, l_{1}, n}^{(1217)}, \bar{G}_{j_{2}, \bar{l}+1, n}^{(1217)}, \bar{G}_{\tilde{j}_{\bar{l}}, n}^{(121)}$, in view of Lemma 6.8 the only nontrivial cases are the following:

$$
\begin{aligned}
& V_{\varpi} \cap V_{\varpi^{\prime}}=\varnothing, \quad \varpi \in \bar{G}_{j_{1}, l_{1}, n}^{(1217)}, \varpi^{\prime} \in \bar{G}_{j_{2}, \bar{l}+1, n}^{(1217)} \\
& V_{\varpi} \cap V_{\varpi^{\prime \prime}}=\varnothing, \quad \varpi \in \bar{G}_{j_{1}, l_{1}, n}^{(7)}, \varpi^{\prime \prime} \in \bar{G}_{\tilde{j}_{\bar{l}}, n}^{(121)} \\
& V_{\varpi^{\prime}} \cap V_{\varpi^{\prime \prime}}=\varnothing, \quad \varpi^{\prime} \in \bar{G}_{j_{2}, \bar{l}+1, n}^{(7)}, \varpi^{\prime \prime} \in \bar{G}_{\tilde{j}_{\bar{l}}, n}^{(121)} .
\end{aligned}
$$

First of all, note that

$$
\bar{G}_{\tilde{j}_{\bar{l}}, n}^{(121)} \cap \bar{G}_{j_{1}, l_{1}, n}^{(7)}=\bar{G}_{\tilde{j}_{\bar{l}}, n}^{(121)} \cap \bar{G}_{j_{2}, \bar{l}+1, n}^{(7)}=\varnothing .
$$

In fact, by Lemma 8.7, if $\varpi \in \bar{G}_{\tilde{j}_{\bar{l}}, n}^{(121)}$, there exists a path connecting $\varpi$ to points in $\bar{G}_{l_{1}, n}^{(4)} \backslash \bar{G}_{j_{1}, n}^{(1)}$ in $\bar{G}_{n}^{(5)} \backslash \bar{G}_{j_{1}, n}^{(1)}$, and, using simply Lemma 6.11 we see that there exists a path connecting $\varpi$ to $\varpi^{\prime \prime} \in \bar{G}_{\bar{l}+1, n}^{(4)}$ contained in $\bar{G}_{n}^{(5)} \backslash \bar{G}_{j_{2}, n}^{(1)}$, and (8.7) now follows from by Remark 6.7. Now, every $\varpi \in \bar{G}_{j_{1}, l_{1}, n}^{(121)}$, by Lemma 6.11 is connected to any $\tilde{\varpi} \in \bar{G}_{j_{1}, l_{1}, n}^{(21)}$ by a path contained in $\bar{G}_{j_{1}, l_{1}, n}^{(1217)}$, thus, in view of Lemma $6.3 \mathrm{i}$ ) and $(8.7)$, contained in $\bar{G}_{n}^{(5)} \backslash \bar{G}_{\tilde{j}_{\bar{l}}, n}^{(121)}$. By Remark 8.6 and Lemma 8.7 we can choose $\tilde{\varpi}$ such that it is connected in $\bar{G}_{n}^{(5)} \backslash \bar{G}_{\tilde{j}_{\bar{l}}, n}^{(121)}$ to some point in $\bar{G}_{\tilde{j}_{\bar{l}}, l_{2}, n}^{(21)}$, where $l_{2}$ is as in Lemma 8.7 iv. Thus, $\varpi$ is connected in $\bar{G}_{n}^{(5)} \backslash \bar{G}_{\tilde{j}_{\bar{l}}, n}^{(121)}$ to some point of $\bar{G}_{\tilde{j}_{\bar{l}}, l_{2}, n}^{(21)}$. Similarly, every $\varpi^{\prime} \in \bar{G}_{j_{2}, \bar{l}+1, n}^{(1217)}$ is connected in $\bar{G}_{n}^{(5)} \backslash \bar{G}_{\tilde{j}_{\bar{l}}, n}^{(121)}$ to some point of $\bar{G}_{\tilde{j}_{\bar{l}}, \bar{l}+1, n}^{(21)}$. Now, (8.4) holds, as in the opposite case, $\varpi$ violates Lemma 8.5. In order to prove (8.5) and (8.6), it suffices to note that, by Remark 6.7, if $\varpi \in \bar{G}_{j_{1}, l_{1}, n}^{(7)}$ (resp. $\varpi^{\prime} \in \bar{G}_{j_{2}, \bar{l}+1, n}^{(7)}$ ) and (8.5) (resp. 8.6) does not hold, then 
$\varpi^{\prime \prime} \in \bar{G}_{j_{1}, l_{1}, n}^{(7)} \cup \bar{G}_{j_{1}, n}^{(1)}$ (resp. $\left.\varpi^{\prime \prime} \in \bar{G}_{j_{2}, \bar{l}+1, n}^{(7)} \cup \bar{G}_{j_{2}, n}^{(1)}\right)$, but this is impossible in view of (8.7) and Lemma 6.3.

Lemma 8.9. Let $\left(j_{1}, j_{2}\right)$ be a $\bar{l}$-pair and let $l_{1}$ and $l_{2}$ be as in Lemma $\left.8.7 \mathrm{iv}\right)$. Then i) The set $\bar{G}_{j_{1}, l_{1}, n}^{(22)}$ is connected and contained in $\bar{G}_{n}^{(5)} \backslash\left(\bar{G}_{j_{1}, l_{1}, n}^{(1217)} \cup \bar{G}_{\tilde{j}_{\bar{l}}, n}^{(121)}\right)$, thus is contained in a component $G_{1}$ of $\bar{G}_{n}^{(5)} \backslash\left(\bar{G}_{j_{1}, l_{1}, n}^{(1217)} \cup \bar{G}_{\tilde{j}_{\bar{l}}, n}^{(121)}\right)$.

$\left.i^{\prime}\right)$ The set $\bar{G}_{j_{2}, \bar{l}+1, n}^{(22)}$ is connected and contained in $\bar{G}_{n}^{(5)} \backslash\left(\bar{G}_{j_{2}, \bar{l}+1, n}^{(121)} \cup \bar{G}_{\tilde{j}_{\bar{l}}, n}^{(121)}\right)$, thus is contained in a component $G_{2}$ of $\bar{G}_{n}^{(5)} \backslash\left(\bar{G}_{j_{2}, \bar{l}+1, n}^{(121)} \cup \bar{G}_{\tilde{j}_{\bar{l}}, n}^{(121)}\right)$.

$\left.i^{\prime \prime}\right) \bar{G}_{\tilde{j}_{\bar{l}}, l_{2}, n}^{(22)}$ is a connected set contained in $\bar{G}_{n}^{(5)} \backslash\left(\bar{G}_{j_{1}, l_{1}, n}^{(1217)} \cup \bar{G}_{\tilde{j}_{\bar{l}}, n}^{(121)}\right)$

$\left.i^{\prime \prime \prime}\right) \bar{G}_{\tilde{j}_{\bar{l}}, \bar{l}+1, n}^{(22)}$ is a connected set contained in $\left.\bar{G}_{n}^{(5)} \backslash \bar{G}_{j_{2}, \bar{l}+1, n}^{(1217)} \cup \bar{G}_{\tilde{j}_{\bar{l}}, n}^{(121)}\right)$

ii) We have

$$
\begin{gathered}
V\left(G_{1}\right) \cap V\left(\bar{G}_{j_{1}, l_{1}, n}^{(1217)}\right)=M_{j_{1}, l_{1}}^{\prime} \\
V\left(G_{1}\right) \cap V\left(\bar{G}_{\tilde{j}_{\bar{l}}, l_{2}, n}^{(121)}\right)=V\left(G_{1}\right) \cap V\left(\bar{G}_{\tilde{j}_{\bar{l}}, n}^{(121)}\right)=M_{\tilde{j}_{\bar{l}}, l_{2}}^{\prime}
\end{gathered}
$$

ii') We have

$$
\begin{gathered}
V\left(G_{2}\right) \cap V\left(\bar{G}_{j_{2}, \bar{l}+1, n}^{(1217)}\right)=M_{j_{2}, \bar{l}+1}^{\prime} \\
V\left(G_{2}\right) \cap V\left(\bar{G}_{\tilde{j}_{\bar{l}}, \bar{l}+1, n}^{(121)}\right)=V\left(G_{2}\right) \cap V\left(\bar{G}_{\tilde{j}_{\bar{l}}, n}^{(121)}\right)=M_{\tilde{j}_{\bar{l}}, \bar{l}+1}^{\prime}
\end{gathered}
$$

iii) The sets $G_{1} \cup \bar{G}_{j_{1}, l_{1}, n}^{(1217)}$ and $G_{2} \cup \bar{G}_{j_{2}, \bar{l}+1, n}^{(1217)}$ are separated.

iv) We have $G_{1} \subseteq \bar{G}_{n}^{(5)} \backslash\left(\bar{G}_{j_{1}, l_{1}, n}^{(1217)} \cup \bar{G}_{\tilde{j}_{\bar{l}}, l_{2}, n}^{(1217)}\right)$

$\left.i v^{\prime}\right)$ We have $G_{2} \subseteq \bar{G}_{n}^{(5)} \backslash\left(\bar{G}_{j_{2}, \bar{l}+1, n}^{(1217)} \cup \bar{G}_{\tilde{j}_{\bar{l}}, \bar{l}+1, n}^{(1217)}\right)$

Proof. Points i) and $i^{\prime}$ ) follow from the definition of the sets involved here and Lemma 6.3. Prove $\left.\mathrm{i}^{\prime \prime}\right)$. The only nontrivial fact to prove is that if $\varpi \in \bar{G}_{\tilde{j}_{\bar{l}}, l_{2}, n}^{(22)}$, then $\varpi \notin \bar{G}_{j_{1}, l_{1}, n}^{(7)}$. Now, there exists a path connecting $\varpi$ to some point of $\bar{G}_{j_{1}, l_{1}, n}^{(22)}$ contained in $\bar{G}_{n}^{(5)} \backslash \bar{G}_{j_{1}, n}^{(1)}$ by Lemma $8.7 \mathrm{iv})$, and by Remark $6.7, \varpi \notin \bar{G}_{j_{1}, l_{1}, n}^{(7)}$. Thus, $\left.\mathrm{i}^{\prime \prime}\right)$ is proved, and $\mathrm{i}^{\prime \prime \prime}$ can be proved similarly.

We now prove ii). In order to prove (8.8), it suffices to note that the inclusion $\subseteq$ follows from the first part of Remark 6.12 and $\supseteq$ holds as $G_{1} \supseteq \bar{G}_{j_{1}, l_{1}, n}^{(22)}$ and $\bar{G}_{j_{1}, l_{1}, n}^{(1217)} \supseteq \bar{G}_{j_{1}, l_{1}, n}^{(21)}$. In order to prove (8.9), we prove

$$
\begin{gathered}
V\left(G_{1}\right) \cap V\left(\bar{G}_{\tilde{j}_{\bar{l}}, l_{2}, n}^{(121)}\right) \subseteq V\left(G_{1}\right) \cap V\left(\bar{G}_{\tilde{j}_{\bar{l}}, n}^{(121)}\right) \\
V\left(G_{1}\right) \cap V\left(\bar{G}_{\tilde{j}_{\bar{l}}, n}^{(121)}\right) \subseteq M_{\tilde{j}_{\bar{l}}, l_{2}}^{\prime}
\end{gathered}
$$




$$
M_{\tilde{j}_{\bar{l}}, l_{2}}^{\prime} \subseteq V\left(G_{1}\right) \cap V\left(\bar{G}_{\tilde{j}_{\bar{l}}, l_{2}, n}^{(121)}\right)
$$

As (8.12) is trivial, we pass to prove (8.13). If $Q \in V\left(G_{1}\right) \cap V\left(\bar{G}_{\tilde{j}_{\bar{l}}, n}^{(121)}\right)$, there exist $\varpi \in G_{1}$, $\varpi^{\prime} \in \bar{G}_{\tilde{j}_{\bar{l}}, n}^{(121)}$ such that

$$
Q \in V_{\varpi} \cap V_{\varpi^{\prime}} \neq \varnothing
$$

and $\varpi \in \bar{G}_{n}^{(5)} \backslash \bar{G}_{\tilde{j}_{\bar{l}}, n}^{(121)}$ is connected to $\varpi^{\prime} \in \bar{G}_{\tilde{j}_{\bar{l}}, s, n}^{(121)}$, in $\bar{G}_{n}^{(5)} \backslash \bar{G}_{\tilde{j}_{\bar{l}}, n}^{(121)}$ for some $s$ such that $\tilde{j}_{\bar{l}} \in \widetilde{B}_{s}^{\prime}$, but by the definition of $G_{1}, \varpi$ is connected by a path contained in $\bar{G}_{n}^{(5)} \backslash \bar{G}_{\tilde{j}_{\bar{l}}, n}^{(121)}$ to a point of $\bar{G}_{j_{1}, l_{1}, n}^{(22)}$ which in turns, by Lemma $8.7 \mathrm{iv)}$ and Remark 8.6, is connected to some point of $\bar{G}_{\tilde{j}_{\bar{l}}, l_{2}, n}^{(121)}$ in $\bar{G}_{n}^{(5)} \backslash\left(\bar{G}_{\tilde{j}_{\bar{l}}, n}^{(121)} \cup \bar{G}_{j_{1}, n}^{(121)}\right)$, thus $\varpi$ is connected in $\bar{G}_{n}^{(5)} \backslash \bar{G}_{\tilde{j}_{\bar{l}}, n}^{(121)}$ to a point of $\bar{G}_{\tilde{j}_{\bar{l}}, l_{2}, n}^{(121)}$, and by Lemma 8.5,s $=l_{2}$, and moreover, by (8.15) and the last part of Remark 6.12, $Q \in M_{\tilde{j}_{\bar{l}}, l_{2}}^{\prime}$, and (8.13) is proved. In order to prove of (8.14), we first prove

$$
V\left(G_{1}\right) \cap V\left(\bar{G}_{\tilde{j}_{\bar{l}}, l_{2}, n}^{(121)}\right) \neq \varnothing
$$

If $\varpi \in \bar{G}_{j_{1}, l_{1}, n}^{(22)}$, then $\varpi \in G_{1}$, and as seen above, $\varpi$ is connected to $\hat{\varpi} \in \bar{G}_{\tilde{j}_{\overline{1}}, l_{2}, n}^{(121)}$ by a path $\left(\varpi_{0}, \ldots, \varpi_{m}\right)$ in $\bar{G}_{n}^{(5)} \backslash\left(\bar{G}_{\tilde{j}_{l}, n}^{(121)} \cup \bar{G}_{j_{1}, n}^{(121)}\right)$. As $\varpi_{0}=\varpi, \ldots, \varpi_{m-1} \in \bar{G}_{n}^{(5)} \backslash \bar{G}_{j_{1}, n}^{(1)}$, and $\varpi_{0} \notin \bar{G}_{j_{1}, l_{1}, n}^{(7)}$, then $\varpi_{0}=\varpi, \ldots, \varpi_{m-1} \notin \bar{G}_{j_{1}, l_{1}, n}^{(7)}$ by Remark 6.7 , hence the path is in $\bar{G}_{n}^{(5)} \backslash\left(\bar{G}_{j_{1}, l_{1}, n}^{(1217)} \cup \bar{G}_{\tilde{j}_{\bar{l}}, n}^{(121)}\right)$, and $\varpi_{m-1} \in G_{1}$, thus there exists

$$
\widetilde{Q} \in V_{\varpi_{m-1}} \cap V_{\hat{\varpi}} \subseteq V\left(G_{1}\right) \cap V\left(\bar{G}_{\tilde{j}_{\bar{l}}, l_{2}, n}^{(121)}\right)
$$

and (8.16) is proved. Note that, by (8.12) and (8.13), we have $\widetilde{Q} \in M_{\tilde{j}_{\bar{l}}, l_{2}}^{\prime}$. Suppose $Q \in M_{\tilde{j}_{\bar{l}}, l_{2}}^{\prime}$. Then, by (8.17) and the definition of $M_{\tilde{j}_{\bar{l}}, l_{2}}^{\prime}$, there exist

$$
\begin{gathered}
\varpi_{(1)} \in G_{1}, \varpi_{(2)} \in \bar{G}_{\tilde{j}_{\bar{l}}, l_{2}, n}^{(121)} \\
\varpi_{(3)} \in \bar{G}_{\tilde{j}_{\bar{l}}, l_{2}, n}^{(22)}, \varpi_{(4)} \in \bar{G}_{\tilde{j}_{\bar{l}}, l_{2}, n}^{(21)} \subseteq \bar{G}_{\tilde{j}_{\bar{l}}, l_{2}, n}^{(121)}
\end{gathered}
$$

such that

$$
\begin{aligned}
& \widetilde{Q} \in V_{\varpi_{(1)}} \cap V_{\varpi_{(2)}} \\
& Q \in V_{\varpi_{(3)}} \cap V_{\varpi_{(4)}}
\end{aligned}
$$

As $\bar{G}_{\tilde{j}_{\bar{l}}, l_{2}, n}^{(22)}$ is a connected set, and by i' $)$, contained in $\bar{G}_{n}^{(5)} \backslash\left(\bar{G}_{j_{1}, l_{1}, n}^{(1217)} \cup \bar{G}_{\tilde{j}_{\bar{l}}, n}^{(121)}\right)$, and by (8.18), (8.20) and Remark 6.12, we have $\varpi_{(1)} \in \bar{G}_{\tilde{j}_{\bar{l}}, l_{2}, n}^{(22)}$, then $\bar{G}_{\tilde{j}_{\bar{l}}, l_{2}, n}^{(22)} \subseteq G_{1}$. Thus, in view 
of (8.19) and (8.21), $Q \in V\left(G_{1}\right) \cap V\left(\bar{G}_{\tilde{j}_{\bar{l}}, l_{2}, n}^{(121)}\right)$, hence $M_{\tilde{j}_{\bar{l}}, l_{2}}^{\prime} \subseteq V\left(G_{1}\right) \cap V\left(\bar{G}_{\tilde{j}_{\bar{l}}, l_{2}, n}^{(121)}\right)$, and (8.14), thus ii) is proved. The proof of $\left.\mathrm{ii}^{\prime}\right)$ is completely analogous to that of ii).

We now prove iii). Note that, by (8.8) and (8.10), the sets $G_{1} \cup \bar{G}_{j_{1}, l_{1}, n}^{(1217)}$ and $G_{2} \cup \bar{G}_{j_{2}, \bar{l}+1, n}^{(1217)}$ are connected. Thus, if $\varpi \in G_{1} \cup \bar{G}_{j_{1}, l_{1}, n}^{(1217)}$, in view of Lemma $8.7 \mathrm{iv)}$ and Remark 8.6, there exists a path connecting $\varpi$ to some point of $\bar{G}_{\tilde{j}_{\bar{l}}, l_{2}, n}^{(21)}$, and if $\varpi^{\prime} \in G_{2} \cup \bar{G}_{j_{2}, \bar{l}+1, n}^{(1217)}$, there exists a path connecting $\varpi^{\prime}$ to some point of $\bar{G}_{\tilde{j}_{\bar{l}}, \bar{l}+1, n}^{(21)}$, and, in view also of Lemma 8.8, both the paths are in $\bar{G}_{n}^{(5)} \backslash \bar{G}_{\tilde{j}_{\bar{l}}, n}^{(121)}$, thus, if $G_{1} \cup \bar{G}_{j_{1}, l_{1}, n}^{(1217)}$ and $G_{2} \cup \bar{G}_{j_{2}, \bar{l}+1, n}^{(1217)}$ are not separated, this contradicts Lemma 8.5, and iii) is proved.

To prove iv), we have only to show that, if $\varpi \in G_{1}$, then $\varpi \notin \bar{G}_{\tilde{j}_{l}, l_{2}, n}^{(7)}$. But, as seen in the proof of iii), there exists a path connecting $\varpi$ to some point $\hat{\varpi}$ of $\bar{G}_{\tilde{j}_{\bar{l}}, l_{2}, n}^{(21)}$, and the path $\left(\varpi_{0}, \ldots, \varpi_{m}\right)$ is in $\bar{G}_{n}^{(5)} \backslash \bar{G}_{\tilde{j}_{\bar{l}}, n}^{(121)}$. In this way all $\varpi_{s} \in \bar{G}_{n}^{(5)}$ for all $s$, and all $\varpi_{s} \notin \bar{G}_{\tilde{j}_{\bar{l}}, n}^{1}$, and, as $\hat{\varpi} \in \bar{G}_{l_{2}, n}^{(4)}$, by Remark $6.7 \varpi \notin \bar{G}_{\tilde{j}_{\bar{l}}, l_{2}, n}^{(7)}$, thus iv) is proved, and $\mathrm{iv}^{\prime}$ ) can be proved in the same way.

We are now ready to prove the reverse-triangular inequality (Lemma 8.10), and then, in Lemma 8.11, we will prove that the effective resistances between $P_{j_{1}}$ and $P_{j_{2}}$ approximates $\widetilde{\bar{R}}_{\left\{j_{1}, j_{2}\right\}}$.

Lemma 8.10. Let $\left(j_{1}, j_{2}\right)$ be a $\bar{l}$-pair and let $l_{1}$ and $l_{2}$ be as in Lemma 8.7 iv).Then

$$
\begin{aligned}
& \widehat{R}\left(S_{r_{(g, \eta, n)}, \mathcal{A}_{\left\{j_{1}, l_{1} ; j_{2}, \bar{l}+1\right\}}}(q)\right)_{M_{j_{1}, l_{1}}^{\prime}, M_{j_{2}, \bar{l}+1}^{\prime}} \geq \\
& \widehat{R}\left(S_{r_{(g, \eta, n)}, \mathcal{A}_{\left\{j_{1}, l_{1} ; \tilde{j}_{\bar{l}}, l_{2}\right\}}}(q)\right)_{M_{j_{1}, l_{1}}^{\prime}, M_{\tilde{j}_{\bar{l}}, l_{2}}^{\prime}}+\widehat{R}\left(S_{r_{(g, \eta, n)}, \mathcal{A}_{j_{2}, \bar{l}+1 ; \tilde{j}_{\bar{l}}, \bar{l}+1}}(q)\right)_{M_{\tilde{j}_{\bar{l}}, \bar{l}+1}^{\prime}, M_{j_{2}, \bar{l}+1}^{\prime}} \\
& \widehat{R}\left(S_{r_{(g, \eta, n)}, \bar{G}_{n}^{(5)}}(q)\right)_{\left\{P_{j_{1}}\right\},\left\{P_{j_{2}}\right\}} \geq \widehat{R}\left(S_{r_{(g, \eta, n)}, \mathcal{A}_{\left\{j_{1}, l_{1} ; j_{2}, \bar{l}+1\right\}}}(q)\right)_{M_{j_{1}, l_{1}}^{\prime}, M_{j_{2}, \bar{l}+1}^{\prime}} \\
& \mathcal{A}_{\left\{j, l ; j^{\prime}, l^{\prime}\right\}}:=\bar{G}_{n}^{(5)} \backslash\left(\bar{G}_{j, l, n}^{(1217)} \cup \bar{G}_{j^{\prime}, l^{\prime}, n}^{(1217)}\right), \quad j \in \widetilde{B}_{l}^{\prime}, j^{\prime} \in \widetilde{B}_{l^{\prime}}^{\prime} .
\end{aligned}
$$

Proof. We use Lemma 3.7 i) with

$\mathcal{B}_{1}=G_{1}, \quad \mathcal{B}_{2}=G_{2}, \quad \mathcal{B}_{3}=\bar{G}_{\tilde{j}_{\bar{l}}, n}^{(121)}, \quad \mathcal{B}_{4}=\bar{G}_{n}^{(5)} \backslash\left(\bar{G}_{\tilde{j}_{\bar{l}}, n}^{(121)} \cup \bar{G}_{j_{1}, l_{1}, n}^{(121)} \cup \bar{G}_{j_{2}, \bar{l}+1, n}^{(1217)} \cup G_{1} \cup G_{2}\right)$

In this way, $\mathcal{B}_{1}, \mathcal{B}_{2}, \mathcal{B}_{3}, \mathcal{B}_{4}$ are mutually disjoint subsets of $\mathcal{A}_{3, n}$ by their definition and Lemma 8.9 iii) Moreover, their union $\mathcal{A}=\mathcal{B}_{1} \cup \mathcal{B}_{2} \cup \mathcal{B}_{3} \cup \mathcal{B}_{4}$ is given by 


$$
\mathcal{A}=\bar{G}_{n}^{(5)} \backslash\left(\bar{G}_{j_{1}, l_{1}, n}^{(1217)} \cup \bar{G}_{j_{2}, \bar{l}+1, n}^{(1217)}\right)=\mathcal{A}_{\left\{j_{1}, l_{1} ; j_{2}, \bar{l}+1\right\}}
$$

In fact, we use Lemma 8.8 and Lemma 8.9 iii) to prove the inclusion $\subseteq$ in (8.24) and the inclusion $\supseteq$ is trivial. Moreover, $\mathcal{B}_{1}, \mathcal{B}_{2}$ are separated by Lemma $\left.8.9 \mathrm{ii}\right)$, and they are separated from $\mathcal{B}_{4}$ by their definition. Thus, we can use Lemma $3.7 \mathrm{i}$ ) with $A_{1}=M_{j_{1}, l_{1}}^{\prime}$, $A_{2}=M_{j_{2}, \bar{l}+1}^{\prime}$, and we have $M_{1}=M_{\tilde{j}_{\bar{l}}, l_{2}}^{\prime}, M_{2}=M_{\tilde{j}_{\bar{l}}, \bar{l}+1}^{\prime}$, and the hypothesis of Lemma 3.7 i) is satisfied also in view of (8.8), (8.9), (8.10), (8.11). We thus obtain

$$
\begin{aligned}
& \widehat{R}\left(S_{r_{(g, \eta, n)}, \mathcal{A}_{\left\{j_{1}, l_{1} ; j_{2}, \bar{l}+1\right\}}}(q)\right)_{M_{j_{1}, l_{1}}^{\prime}, M_{j_{2}, \bar{l}+1}^{\prime}} \geq \\
& \widehat{R}\left(S_{r_{(g, \eta, n)}, G_{1}}(q)\right)_{M_{j_{1}, l_{1}}^{\prime}, M_{\tilde{j}_{\bar{l}}, l_{2}}^{\prime}}+\widehat{R}\left(S_{r_{(g, \eta, n)}, G_{2}}(q)\right)_{M_{\tilde{j}_{\bar{l}}, \bar{l}+1}^{\prime}, M_{j_{2}, \bar{l}+1}^{\prime}} \geq \\
& \widehat{R}\left(S_{r_{(g, \eta, n)}, \mathcal{A}_{\left\{j_{1}, l_{1} ; \tilde{j}_{\bar{l}}, l_{2}\right\}}}(q)\right)_{M_{j_{1}, l_{1}}^{\prime}, M_{\tilde{j}_{\bar{l}}, l_{2}}^{\prime}}+\widehat{R}\left(S_{r_{(g, \eta, n)}, \mathcal{A}_{j_{2}, \bar{l}+1 ; \tilde{j}_{\bar{l}}, \bar{l}+1}}(q)\right)_{M_{\tilde{j}_{\bar{l}}, \bar{l}+1}^{\prime}, M_{j_{2}, \bar{l}+1}^{\prime}}
\end{aligned}
$$

where the second inequality holds by Lemma 8.9, iv) and $\left.\mathrm{iv}^{\prime}\right)$, and Remark 3.6. We have thus proved (8.22) and we are going to prove (8.23). Given $v \in \mathcal{L}_{V\left(\mathcal{A}_{j_{1}, l_{1} ; j_{2}, \bar{l}+1}\right), M_{j_{1}, l_{1}}^{\prime}, M_{j_{2}, \bar{l}+1}^{\prime}}$ we define $\tilde{v} \in \mathcal{L}_{V\left(\bar{G}_{n}^{(5)}\right),\left\{P_{j_{1}}\right\},\left\{P_{j_{2}}\right\}}$, which extends $v$ on $V\left(\bar{G}_{n}^{(5)}\right)$ putting $\tilde{v}=0$ on $V\left(\bar{G}_{j_{1}, l_{1}, n}^{(1217)}\right)$ and $\tilde{v}=1$ on $V\left(\bar{G}_{j_{2}, \bar{l}+1, n}^{(1217)}\right)$. Note that $\tilde{v}$ is well-defined as, by Lemma 8.8, the sets $V\left(\bar{G}_{j_{1}, l_{1}, n}^{(1217)}\right)$ and $V\left(\bar{G}_{j_{2}, \bar{l}+1, n}^{(1217)}\right)$ are disjoint, and as, by Remark 6.12 ,

$$
V\left(\bar{G}_{j_{1}, l_{1}, n}^{(1217)}\right) \cap V\left(\mathcal{A}_{\left\{j_{1}, l_{1} ; j_{2}, \bar{l}+1\right\}}\right) \subseteq M_{j_{1}, l_{1}}^{\prime}, \quad V\left(\bar{G}_{j_{2}, \bar{l}+1, n}^{(1217)}\right) \cap V\left(\mathcal{A}_{\left\{j_{1}, l_{1} ; j_{2}, \bar{l}+1\right\}}\right) \subseteq M_{j_{2}, \bar{l}+1}^{\prime} .
$$

We have $S_{r_{(g, \eta, n)}, \bar{G}_{n}^{(5)}}^{\prime}(q)(\tilde{v})=S_{r_{(g, \eta, n)}, \mathcal{A}_{\left\{j_{1}, l_{1} ; j_{2}, \bar{l}+1\right\}}^{\prime}}^{\prime}(q)(v)$, and (8.23) immediately follows from the definition of $\widehat{R}$.

We say that $g$ as above is $(n, \eta, q)$-normal, $q \in \widetilde{K}$, if it satisfies the hypothesis of Lemma 8.4, that is, if $g=\tilde{t}_{1, n}$ on $\bar{C}_{j}^{(1)}, g=\tilde{t}_{2, n}$ on $\bar{C}_{j, l}^{(21)}, g=\tilde{t}_{3, n}$ on $\bar{C}_{l}^{(3)}$ for every $l=1, \ldots, h^{\prime}$, $g=t_{2, q, j, l, n}$ on $\bar{C}_{j, l}^{(22)}$. In this way we can formulate Lemma 8.4 in the following way:

For every $\left.\eta \in] 0, \min \left\{\frac{1}{\#\left(\widetilde{B}_{l}^{\prime}\right)}\right\}\right], n \geq n_{1, \eta}$ and $q \in \widetilde{K}$, if $g$ is $(n, \eta, q)$-normal, then for every $l=1, \ldots, h^{\prime}, j_{1}, j_{2} \in \widetilde{B}_{l}^{\prime}$ with $j_{1} \neq j_{2}$

i) $\frac{2}{\#\left(\widetilde{B}_{l}^{\prime}\right)}-3 \eta<\widehat{R}\left(S_{r_{(g, \eta, n)}, \bar{G}_{n}^{(5)}}(q)\right)_{\left\{P_{j_{1}}, P_{j_{2}}\right\}}<\frac{2}{\#\left(\widetilde{B}_{l}^{\prime}\right)}+3 \eta$

ii) $\widehat{R}\left(S_{r_{(g, \eta, n)}, \mathcal{A}_{\left\{j_{1}, l ; j_{2}, l\right\}}}(q)\right)_{\left\{M_{j_{1}, l}^{\prime}, M_{j_{2}, l}^{\prime}\right\}} \geq \frac{2}{\#\left(\widetilde{B}_{l}^{\prime}\right)}-\eta$

Lemma 8.11. There exists $H_{1}>0$, independent of $\eta, n$, such that, for every $\eta \in$ ] $\left.0, \min \left\{\frac{1}{\#\left(\widetilde{B}_{l}^{\prime}\right)}\right\}\right], n \geq n_{1, \eta}$ and $q \in \widetilde{K}$, if $g$ is $(n, \eta, q)$-normal, then for every $j_{1}, j_{2}=$ $1, \ldots, N, j_{1} \neq j_{2}$ we have 


$$
\widetilde{\bar{R}}_{j_{1}, j_{2}}-H_{1} \eta<\widehat{R}\left(S_{r_{(g, \eta, n)}, \bar{G}_{n}^{(5)}}(q)\right)_{\left\{P_{j_{1}}\right\},\left\{P_{j_{2}}\right\}}<\widetilde{\bar{R}}_{j_{1}, j_{2}}+H_{1} \eta
$$

Proof. We prove by recursion that, if $j_{1}, j_{2} \in \bigcup_{l=1}^{\bar{l}} \widetilde{B}_{l}^{\prime}, \bar{l}=1, \ldots, h^{\prime}$, then

$$
\begin{gathered}
\widehat{R}\left(S_{r_{(g, \eta, n)}, \bar{G}_{n}^{(5)}}(q)\right)_{\left\{P_{j_{1}}\right\},\left\{P_{j_{2}}\right\}}<\widetilde{\bar{R}}_{j_{1}, j_{2}}+H_{2, \bar{l}} \eta \\
\left\{\begin{array}{l}
\exists l_{1}, l_{2}=1, \ldots, \bar{l}:\left(j_{1}, l_{1}\right),\left(j_{2}, l_{2}\right) \text { are } \bar{l} \text { - related, } \\
\widetilde{\widetilde{R}}_{j_{1}, j_{2}}-H_{3, \bar{l}} \eta<\widehat{R}\left(S_{\left.r_{(g, \eta, n)}, \mathcal{A}_{\left\{j_{1}, l_{1} ; j_{2}, l_{2}\right\}}(q)\right)_{M_{j_{1}, l_{1}}^{\prime}, M_{j_{2}, l_{2}}^{\prime}}} \widetilde{\widetilde{R}}_{j_{1}, j_{2}}-H_{4, \bar{l}} \eta<\widehat{R}\left(S_{r_{(g, \eta, n)}, \bar{G}_{n}^{(5)}}(q)\right)_{\left\{P_{j_{1}}\right\},\left\{P_{j_{2}}\right\}}\right.
\end{array}\right.
\end{gathered}
$$

The case $\bar{l}=h^{\prime}$ gives (8.25). Note that, if $j_{1}, j_{2}$ lie in a common $\widetilde{B}_{l}^{\prime}$, then (8.26) and (8.27) hold with $H_{2, \bar{l}}=3, H_{3, \bar{l}}=2, H_{4, \bar{l}}=3$ and $l_{2}=l_{1}=l$ by Lemmas 8.4, $\left.8.7 \mathrm{i}\right)$ and $7.6 \mathrm{i}$ ). Hence, (8.26) and (8.27) hold if $\bar{l}=1$. Suppose (8.26) and (8.27) hold for $\bar{l}<h^{\prime}$ and prove it holds for $\bar{l}+1$. Given $j_{1}, j_{2} \in \bigcup_{l=1}^{\bar{l}+1} \widetilde{B}_{l}^{\prime}$, by Lemma 7.5, either $j_{1}, j_{2} \in \widetilde{B}_{\bar{l}+1}^{\prime}$ and (8.26), (8.27) hold as seen above, or $j_{1}, j_{2} \in \bigcup_{l=1}^{\bar{l}} \widetilde{B}_{l}^{\prime}$, and (8.26) and (8.27) hold with $H_{i, \bar{l}+1}=H_{i, \bar{l}}$, for $i=1,2,3$, or one of $\left(j_{1}, j_{2}\right),\left(j_{2}, j_{1}\right)$ is an $\bar{l}$-pair. In the last case, suppose for example $\left(j_{1}, j_{2}\right)$ is an $\bar{l}$-pair. Then, by the recursive hypothesis, we have

$\widehat{R}\left(S_{r_{(g, \eta, n)}, \bar{G}_{n}^{(5)}}^{(q)}(q)\right)_{\left\{P_{j_{1}}\right\},\left\{P_{\bar{j}_{\bar{l}}}\right\}}<\widetilde{\bar{R}}_{j_{1}, \tilde{j}_{\bar{l}}}+H_{2, \bar{l}} \eta, \quad \widehat{R}\left(S_{r_{(g, \eta, n)}, \bar{G}_{n}^{(5)}}(q)\right)_{\left\{P_{\bar{j}_{\bar{l}}}\right\},\left\{P_{j_{2}}\right\}}<\widetilde{\bar{R}}_{\tilde{j}_{\bar{l}}, j_{2}}+3 \eta$ and, as $\widehat{R}\left(S_{r_{(g, \eta, n)}, \bar{G}_{n}^{(5)}}(q)\right)$ is a metric on $V\left(\bar{G}_{n}^{(5)}\right)$, and by Lemma $\left.7.6 \mathrm{ii}\right),(8.26)$ holds with $H_{2, \bar{l}+1}=H_{2, \bar{l}}+3$. In conclusion, (8.26) holds for $\bar{l}+1$ in any case. Moreover, by the recursive hypothesis (8.27) holds with $\tilde{j}_{\bar{l}}$ in place of $j_{2}$, and Lemma 8.7 i) with $\tilde{j}_{\bar{l}}$ in place of $j_{1}$ and $\bar{l}+1$ in place of $l$, in view of Lemma $\left.8.7 \mathrm{ii}\right),\left(j_{1}, l_{1}\right)$ and $\left(j_{2}, \bar{l}+1\right)$ are $(\bar{l}+1)$-related, and, by (8.22) and (8.23) we have

$$
\begin{gathered}
\widehat{R}\left(S_{r_{(g, \eta, n)}, \bar{G}_{n}^{(5)}}(q)\right)_{\left\{P_{j_{1}}\right\},\left\{P_{j_{2}}\right\}} \geq \widehat{R}\left(S_{r_{(g, \eta, n)}, \mathcal{A}_{\left\{j_{1}, l_{1} ; j_{2}, \bar{l}+1\right\}}}(q)\right)_{M_{j_{1}, l_{1}, M_{j_{2}, \bar{l}+1}^{\prime}}^{\prime} \geq} \geq \\
\widehat{R}\left(S_{r_{(g, \eta, n)}, \mathcal{A}_{\left\{j_{1}, l_{1} ; \tilde{j}_{\bar{l}}, l_{2}\right\}}}(q)\right)_{M_{j_{1}, l_{1}}^{\prime}, M_{\bar{j}_{\bar{l}}, l_{2}}^{\prime}}+\widehat{R}\left(S_{r_{(g, \eta, n)}, \mathcal{A}_{j_{2}, \bar{l}+1 ; \tilde{j}_{\bar{l}}, \bar{l}+1}}(q)\right)_{M_{\tilde{j}_{\bar{l}}, \bar{l}+1}^{\prime}, M_{j_{2}, \bar{l}+1}^{\prime}} \\
>\widetilde{\bar{R}}_{j_{1}, \tilde{j}_{\bar{l}}}-H_{3, \bar{l}} \eta+\widetilde{\bar{R}}_{\tilde{j}_{\bar{l}}, j_{2}}-2 \eta=\widetilde{\bar{R}}_{j_{1}, j_{2}}-\left(H_{3, \bar{l}}+2\right) \eta
\end{gathered}
$$

and (8.27) holds for $\bar{l}+1$, with $\bar{l}+1$ in place of $l_{2}, H_{4, \bar{l}+1}=H_{3, \bar{l}+1}=H_{3, \bar{l}}+2$. 
We will now prove that the map $q \mapsto \widehat{\Lambda}\left(r_{(g, \eta, n)}, q\right)$, using the following Lemma.

Lemma 8.12. There exist $\eta>0$ and $n_{2, \eta} \geq n_{1, \eta}$ such that, if $n \geq n_{2, \eta}$ and $q \in \widetilde{K}$, and $g$ is $(n, \eta, q)$-normal, and $g(\varpi)=\frac{\bar{t}}{\eta}$ for every $\varpi \in \bar{C}^{(6)}$, then $\bar{R}\left(\widehat{\Lambda}\left(r_{(g, \eta, n)}, q\right)\right) \in \bar{U}$.

Proof. By Lemma 3.3 we have

$$
\bar{R}\left(\widehat{\Lambda}\left(r_{(g, \eta, n)}, q\right)\right)_{\left\{j_{1}, j_{2}\right\}}=\widehat{R}\left(S_{r_{(g, \eta, n)}, \mathcal{A}_{3, n}}(q)\right)_{\left\{P_{j_{1}}\right\},\left\{P_{j_{2}}\right\}}
$$

On the other hand, putting $\tilde{g}=\frac{\eta}{\bar{t}} g$, we can write $r_{(g, \eta, n)}$ as

$$
\begin{gathered}
r_{(g, \eta, n)}\left(\varpi, \varpi^{\prime \prime}\right)=\tilde{g}(\varpi) r_{\varpi}^{\prime \prime}\left(\varpi^{\prime \prime}\right), \\
r_{\varpi}^{\prime \prime}\left(\varpi^{\prime \prime}\right):= \begin{cases}\frac{\bar{t}^{2}}{\eta^{2} \tilde{t}} & \text { if }\left(\varpi, \varpi^{\prime \prime}\right)=j_{(n)}, \quad \varpi \in \mathcal{A}_{3, n_{2}}, \varpi^{\prime \prime} \in \mathcal{A}_{3, n-n_{2}} \\
\frac{\bar{t}}{\eta} & \text { otherwise }\end{cases}
\end{gathered}
$$

By Lemma 7.10 we have

$$
\begin{aligned}
& \widehat{R}\left(S_{r_{(g, \eta, n)}, \mathcal{A}_{3, n}}(q)\right)_{\left\{P_{j_{1}}\right\},\left\{P_{j_{2}}\right\}}=\widehat{R}\left(S_{\tilde{g}, \mathcal{A}_{3, n_{2}}}\left(\widehat{\Lambda}_{r_{\varpi}^{\prime \prime}(q)}\right)\right)_{\left\{P_{j_{1}}\right\},\left\{P_{j_{2}}\right\}}, \\
& \widehat{R}\left(S_{\left.r_{(g, \eta, n)}, \bar{G}_{n}^{(5)}(q)\right)_{\left\{P_{j_{1}}\right\},\left\{P_{j_{2}}\right\}}}=\widehat{R}\left(S_{\tilde{g}, \bar{C}^{(5)}}\left(\widehat{\Lambda}_{r_{\varpi}^{\prime \prime}}(q)\right)\right)_{\left\{P_{j_{1}}\right\},\left\{P_{j_{2}}\right\}} .\right.
\end{aligned}
$$

Moreover, by Lemma 3.9, as $\mathcal{A}_{3, n_{2}}=\bar{C}^{(5)} \cup \bar{C}^{(6)}$, we have

$$
\begin{gathered}
0 \leq(1 / \widehat{R})\left(S_{\tilde{g}, \mathcal{A}_{3, n_{2}}}\left(\widehat{\Lambda}_{r_{\varpi}^{\prime \prime}}(q)\right)\right)_{\left\{P_{j_{1}}\right\},\left\{P_{j_{2}}\right\}}-(1 / \widehat{R})\left(S_{\tilde{g}, \bar{C}^{(5)}}\left(\widehat{\Lambda}_{r_{\varpi}^{\prime \prime}}(q)\right)\right)_{\left\{P_{j_{1}}\right\},\left\{P_{j_{2}}\right\}} \\
\leq k^{n_{2}} \max \left\{\left|\widehat{\Lambda}_{r_{\varpi}^{\prime \prime}}(q)\right|: \varpi \in \bar{C}^{(6)}\right\}=k^{n_{2}} \frac{\bar{t}}{\eta}\left|\widehat{\Lambda}_{1}^{n-n_{2}}(q)\right|_{n \rightarrow+\infty} 0
\end{gathered}
$$

uniformly for $q \in \widetilde{K}$ by Corollary 7.9. In fact, when $\varpi \in \bar{C}^{(6)}$ we have $r_{\varpi}^{\prime \prime}=\frac{\bar{t}}{\eta}$ and, by (7.2), $\widehat{\Lambda}_{1 ; n-n_{2}}=\widehat{\Lambda}_{1}^{n-n_{2}}$. Since the quantity $\widehat{R}\left(S_{\tilde{g}, \bar{C}^{(5)}}\left(\widehat{\Lambda}_{r_{\varpi}^{\prime \prime}}(q)\right)\right)_{\left\{P_{j_{1}}\right\},\left\{P_{j_{2}}\right\}}$, by Lemma 8.11 and (8.29), is uniformly bounded, we have

$$
\left|\widehat{R}\left(S_{\tilde{g}, \mathcal{A}_{3, n_{2}}}\left(\widehat{\Lambda}_{r_{\varpi}^{\prime \prime}}(q)\right)\right)_{\left\{P_{j_{1}}\right\},\left\{P_{j_{2}}\right\}}-\widehat{R}\left(S_{\tilde{g}, \bar{C}^{(5)}}\left(\widehat{\Lambda}_{r_{\varpi}^{\prime \prime}}(q)\right)\right)_{\left\{P_{j_{1}}\right\},\left\{P_{j_{2}}\right\}}\right|<\eta
$$

for sufficiently large $n$, say $n \geq n_{2, \eta}$. Thus, by Lemma 8.11, (8.28), (8.29) and (8.30), putting $\bar{r}=r_{(g, \eta, n)}$, for such $n$, we have

$$
\begin{aligned}
& \left|\bar{R}(\widehat{\Lambda}(\bar{r}, q))_{\left\{j_{1}, j_{2}\right\}}-\widetilde{\bar{R}}_{\left\{j_{1}, j_{2}\right\}}\right| \leq \\
& \left|\bar{R}(\widehat{\Lambda}(\bar{r}, q))_{\left\{j_{1}, j_{2}\right\}}-\widehat{R}\left(S_{\bar{r}, \bar{G}_{n}^{(5)}}(q)\right)_{\left\{P_{j_{1}}\right\},\left\{P_{j_{2}}\right\}}\right|+\left|\widehat{R}\left(S_{\bar{r}, \bar{G}_{n}^{(5)}}(q)\right)_{\left\{P_{j_{1}}\right\},\left\{P_{j_{2}}\right\}}-\widetilde{\bar{R}}_{\left\{j_{1}, j_{2}\right\}}\right| \\
& <\left|\bar{R}(\widehat{\Lambda}(\bar{r}, q))_{\left\{j_{1}, j_{2}\right\}}-\widehat{R}\left(S_{\bar{r}, \bar{G}_{n}^{(5)}}(q)\right)_{\left\{P_{j_{1}}\right\},\left\{P_{j_{2}}\right\}}\right|+H_{1} \eta
\end{aligned}
$$


$=\left|\widehat{R}\left(S_{\tilde{g}, \mathcal{A}_{3, n_{2}}}\left(\widehat{\Lambda}_{r_{\varpi}^{\prime \prime}}(q)\right)\right)_{\left\{P_{j_{1}}\right\},\left\{P_{j_{2}}\right\}}-\widehat{R}\left(S_{\tilde{g}, \bar{C}^{(5)}}\left(\widehat{\Lambda}_{r_{\varpi}^{\prime \prime}}(q)\right)\right)_{\left\{P_{j_{1}}\right\},\left\{P_{j_{2}}\right\}}\right|+H_{1} \eta<\left(H_{1}+1\right) \eta$.

So far, we have kept $\eta$ fixed. Now, for sufficiently small $\eta$ we have $\bar{R}\left(\widehat{\Lambda}\left(r_{(g, \eta, n)}, q\right)\right) \in \bar{U}$.

Corollary 8.13. Put $\widetilde{W}^{n}:=\{r \in] 0,+\infty\left[\mathcal{A}_{3, n}: r_{j_{(n)}} \leq r_{\varpi} \forall j=1, \ldots, N, \varpi \in \mathcal{A}_{3, n}\right\}$. Then for sufficiently large $n$ there exists a continuous function $\bar{r}: \widetilde{K} \rightarrow \widetilde{W}^{n}$ such that, for every $q \in \widetilde{K}$, we have $\widehat{\Lambda}(\bar{r}(q), q) \in \widetilde{K}$.

Proof. Let $\eta$ be as in Lemma 8.12 and $n \geq n_{2, \eta}$. For every $q \in \widetilde{K}$ define $\left.g_{q}: \mathcal{A}_{3, n_{2}} \rightarrow\right] 0,+\infty[$ by

$$
g_{q}(\varpi)= \begin{cases}\tilde{t}_{1, n} & \text { if } \varpi \in \bar{C}_{j}^{(1)} \\ \tilde{t}_{2, n} & \text { if } \varpi \in \bar{C}_{j, l}^{(21)} \\ t_{2, q, j, l, n} & \text { if } \varpi \in \bar{C}_{j, l}^{(22)} \\ \tilde{t}_{3, n} & \text { if } \varpi \in \bar{C}_{l}^{(3)} \\ \frac{\bar{t}}{\eta} & \text { if } \varpi \in \bar{C}^{(6)}\end{cases}
$$

Let $\bar{r}(q)=r_{\left(g_{q}, \eta\right)}$ for every $q \in \widetilde{K}$. We easily see that $\bar{r}$ is continuous and takes values in $\widetilde{W}^{n}$. Moreover, $g_{q}$ is $(n, \eta, q)$-normal for every $q \in \widetilde{K}$, so that, by Lemma 8.12 , we have $\bar{R}(\widehat{\Lambda}(\bar{r}(q), q)) \in \bar{U}$, and therefore, by Lemma $7.7, \widehat{\Lambda}(\bar{r}(q), q) \in \widetilde{K}$ for every $q \in \widetilde{K}$.

Theorem 8.14. For sufficiently large $n$, there exists a regular G-eigenform on $\mathcal{F}_{n}$.

Proof. The map $q \mapsto \widehat{\Lambda}(\bar{r}(q), q)$ is continuous from the nonempty convex compact $\widetilde{K}$ into itself, thus it has a fixed point $q_{1}$. In other words there exists $q_{1} \in \widetilde{K} \subseteq \widetilde{\mathcal{Q}}$ such that

$$
q_{1}=\widehat{\Lambda}\left(\bar{r}\left(q_{1}\right), q_{1}\right)=\widehat{\Lambda}_{\bar{r}\left(q_{1}\right)}\left(q_{1}\right)
$$

Taking $E=\bar{I}\left(q_{1}\right)$, we have $\Lambda_{\bar{r}\left(q_{1}\right)}(E)=E$, so that $E$ is a G-eigenform. On the other hand, as $r:=\bar{r}\left(q_{1}\right) \in \tilde{W}^{n}$, the G-eigenform $E$ is regular by Remark 4.4.

\section{References}

[1] B.M. Hambly, V. Metz, A. Teplyaev, Self-Similar Energies on Post-critically Finite Self-Similar Fractals, J. London Math. Soc. 74 (2006) 93-112.

[2] K. Hattori, T. Hattori, H. Watanabe, Gaussian Field Theories on General Networks and the Spectral Dimension, Progr. Theor. Phys. Suppl. 92 (1987), 108-143.

[3] J. Kigami, Harmonic Calculus on p.c.f. Self-similar Sets, Trans. Amer. Math. Soc. 335 (1993), 721-755.

[4] J. Kigami, Analysis on fractals, Cambridge Tracts in Mathematics, 143. Cambridge University Press, Cambridge, 2001. 
[5] T. Lindstrøm, Brownian Motion on Nested Fractals, Mem. Amer. Math. Soc. No. 420 (1990).

[6] V.Metz, The short-cut test, Journal of Functional Analysis 220 (2005), 118-156.

[7] R. Peirone, Existence of Eigenforms on Fractals with three Vertices, Proc. Royal Soc. Edinburgh, 137 A (2007), 1073-1080.

[8] R. Peirone, Existence of Eigenforms on Nicely Separated Fractals, in Proceedings of Symposia in Pure Mathematics, Amer. Math. Soc., Vol. 77, 231-241, 2008

[9] C. Sabot, Existence and Uniqueness of Diffusions on Finitely Ramified Self-Similar Fractals, Ann. Sci. École Norm. Sup. (4) 30 (1997), no. 5, 605-673.

[10] R. S. Strichartz, Differential equations on fractals: A tutorial, Princeton University Press, 2006 